\title{
Low Order Solutions to the Perfect Regulation Problem with BOUNDED PEAKING
}

by

\author{
Zhaowei Wang
}

A thesis submitted in conformity with the requirements for the degree of Master of Applied Science

Graduate Department of Electrical \& Computer Engineering University of Toronto

(c) Copyright 2015 by Zhaowei Wang 


\section{Abstract \\ Low Order Solutions to the Perfect Regulation Problem with Bounded Peaking \\ Zhaowei Wang \\ Master of Applied Science \\ Graduate Department of Electrical \& Computer Engineering \\ University of Toronto}

2015

High gain control is an intrinsic aspect of high performance systems. The perfect regulation problem with bounded peaking refers to regulating a system arbitrarily fast, while maintaining boundedness at the output. In this thesis, we construct a family of controllers of low dynamic order parameterized by a gain parameter which solves this problem for multivariable, linear time-invariant systems which are invertible and minimum phase. In particular we obtain a lower bound on the order of the controller which is only a function of a structural property of the system known as the orders of infinite zeros. A further development shows how, under certain conditions, the techniques developed can be used to design decentralized controllers which also solve the perfect regulation problem with bounded peaking. 
To my parents. 


\section{Contents}

$\begin{array}{lll}1 & \text { Introduction } & 1\end{array}$

1.1 High Gain Control Systems $\ldots \ldots \ldots \ldots \ldots \ldots \ldots$

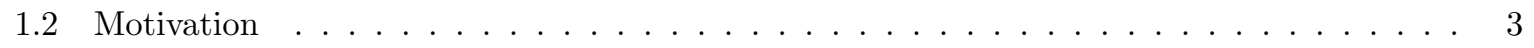

1.3 Literature Review . . . . . . . . . . . . . . . . . . . . . . . 4

1.4 Thesis Statement \& Contributions $\ldots \ldots \ldots \ldots \ldots \ldots \ldots$

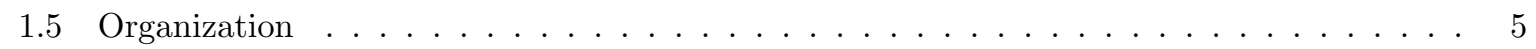

1.6 Problem Formulation $\ldots \ldots \ldots \ldots \ldots \ldots$

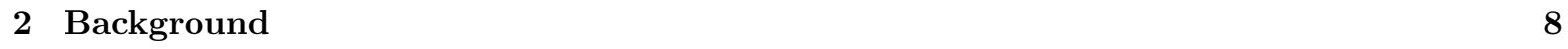

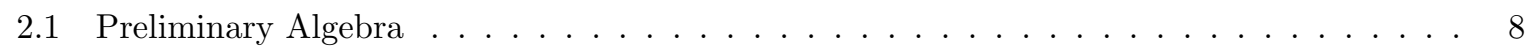

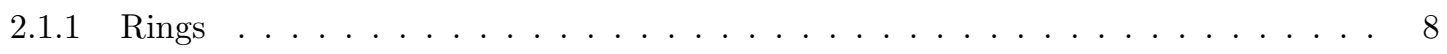

$2.1 .2 \quad$ Matrix Rings \& Canonical Forms $\ldots \ldots \ldots \ldots \ldots \ldots$

2.1 .3 The Ring of Transfer Matrices \& Infinite Zeros . . . . . . . . . . . . . . . . . . 12

2.2 Multivariable Root Locus . . . . . . . . . . . . . . . . . . . . . . . . 13

2.2 .1 The Standard SISO Root Locus Problem . . . . . . . . . . . . . . . . . . . 13

2.2 .2 Multivariable Generalizations . . . . . . . . . . . . . . . . . . . . 14

2.3 Cheap Control \& Perfect Regulation with Bounded Peaking . . . . . . . . . . . . . . . . . 19

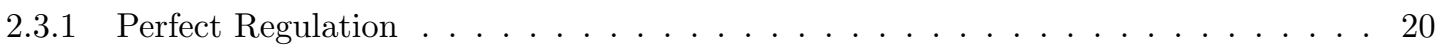

2.3 .2 Bounded Peaking . . . . . . . . . . . . . . . . . . . . . . . . . . . . . 21

$2.3 .3 \quad$ A Characterization of Perfect Regulation with Bounded Peaking . . . . . . . . . . 21

3 High Gain Stabilization for Single-Input Single-Output Systems 23

3.1 Behaviour of the Finite Poles of the Closed-loop System ～. . . . . . . . . . . . . . . . . 24

3.2 Asymptotic Behaviour of the Closed-loop System ～. . . . . . . . . . . . . . . . . . 24

$3.2 .1 \quad$ A Motivating Example . . . . . . . . . . . . . . . . . . . . . . . . 25

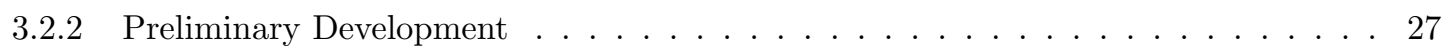

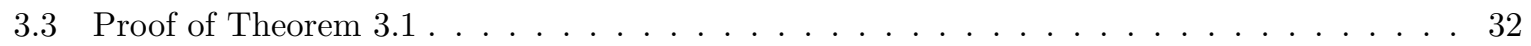

3.4 Further Developments $\ldots \ldots \ldots \ldots \ldots \ldots \ldots$

$3.5 \quad$ A Lower Bound on the Order of the Controller . . . . . . . . . . . . . . . . . . . 37

\begin{tabular}{|lll}
\hline & Perfect Regulation with Bounded Peaking & 38
\end{tabular}

4.1 On The Smith-McMillan Form $\ldots \ldots \ldots \ldots$. . . . . . . . . . . . . . . . . . . 38

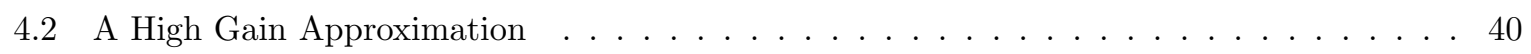

4.3 Simple Null Structure $\ldots \ldots \ldots \ldots \ldots \ldots \ldots$ 
4.4 High Gain Stabilization, the MIMO Case . . . . . . . . . . . . . . . . . . . . . . . . . . . 51

4.5 Perfect Regulation with Bounded Peaking . . . . . . . . . . . . . . . . . . . . . . . . . . 52

$4.6 \quad$ Decentralized Control \& Perfect Regulation . . . . . . . . . . . . . . . . . . . . . . . . . . 53

4.6 .1 Positive Instantaneous Gain . . . . . . . . . . . . . . . . . . . . . . . . . . . 54

$4.6 .2 \quad$ Perfect Decentralized Regulation with Bounded Peaking . . . . . . . . . . . . . . . 54

\begin{tabular}{|lll}
5 & Case Studies & 56
\end{tabular}

5.1 Industrial Chemical Plant . . . . . . . . . . . . . . . . . . . . . . . . . . . . . . . . . . 56

5.2 An Example in the Gap . . . . . . . . . . . . . . . . . . . . . . . . . . . . . . . . . . . . 58

5.3 A Violation of Simple Null Structure . . . . . . . . . . . . . . . . . . . . . . . . . . . . . . 59

5.4 Designing For the Orders of Infinite Zeros . . . . . . . . . . . . . . . . . . . . . . . . . . . 60

5.5 Decentralized Control of 2-Linked Inverted Pendulum . . . . . . . . . . . . . . . . . . . . 61

\begin{tabular}{|lll}
\hline Concluding Remarks & 64 \\
\hline
\end{tabular}

6.1 Summary of Results . . . . . . . . . . . . . . . . . . . . . . . . . 64

6.2 Future Work . . . . . . . . . . . . . . . . . . . . . . . . . 64

6.3 Final Thoughts . . . . . . . . . . . . . . . . . . . . . . . . . . 65

\begin{tabular}{|l|l|}
\hline A A generalization of Rouché's Theorem & 66
\end{tabular}

\begin{tabular}{|l}
\hline Bibliography \\
\hline
\end{tabular} 


\section{List of Figures}

1.1 A high gain control system $\ldots \ldots \ldots \ldots \ldots \ldots \ldots \ldots$

1.2 An example of high gain control $\ldots \ldots \ldots \ldots \ldots \ldots \ldots$

1.3 Step response of example system as $k$ is increased. . . . . . . . . . . . . . . . . . . 2

1.4 An example where high gain control fails $\ldots \ldots \ldots \ldots \ldots$

2.1 The SISO root locus setup $\ldots \ldots \ldots \ldots \ldots \ldots \ldots \ldots$

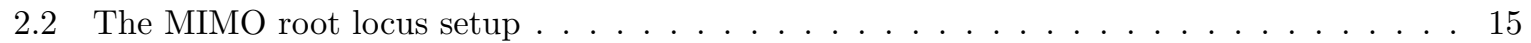

3.1 Newton-Puiseux diagram for the standard root locus formulation . . . . . . . . . . . 26

3.2 Newton-Puiseux diagram for $\Delta(g, z), r^{*}=5 \ldots \ldots \ldots \ldots \ldots \ldots$

5.1 Initial condition response of chemical plant. Note that our choice of gains were not large enough to significantly change the response in the fourth output. . . . . . . . . . . 57

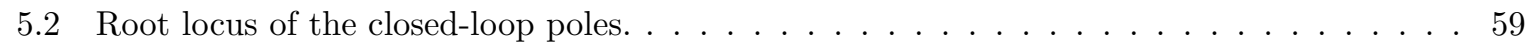

$5.3 \quad$ Initial condition response for the decentralized control of 2-linked inverted pendulum. . . 62 


\section{Chapter 1}

\section{Introduction}

One of the fundamental principles of engineering is that nothing can be attained for free. Engineering design is then the art of managing the trade-offs between various system requirements in order to meet the designer's specifications. For the control engineer, the most common situation is to ensure satisfactory performance of the system while minimizing the cost of actuation, and complexity of the controller. The entirety of this thesis is devoted to the construction of "simple" controllers for which "arbitrarily good" performance can be achieved at the cost of high gain actuation. These terms are to be made precise in the developments to follow.

We introduce in this chapter the notion of high gain control, and give the motivation for the results presented in this thesis. A brief literature review is conducted and details some of the classical approaches to high gain control. The remaining sections present the thesis statement, and contributions, as well as the organization of the remaining chapters.

\subsection{High Gain Control Systems}

Consider the following control system in Figure 1.1

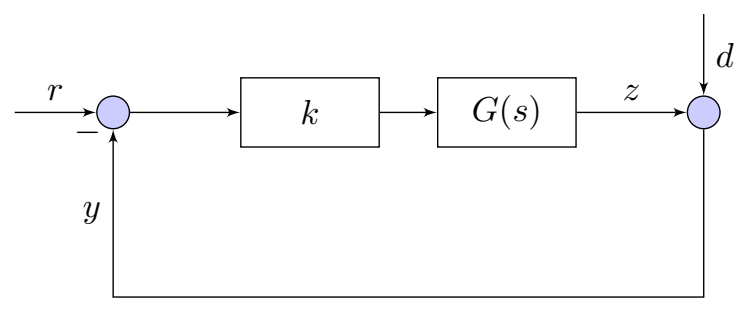

Figure 1.1: A high gain control system

$G(s)$ is the lumped, frequency domain representation of a single-input single-output (SISO), linear timeinvariant system, $z$ is the output of the plant, and $d$ is an additive disturbance at the output. The control is given by $u=k(r-y)$. Suppose at the moment our goal is to analyze the tracking, disturbance rejection, and robustness properties of the closed-loop system. 
The transfer function from $r$ to $y$, and from $d$ to $y$ are given by

$$
T_{r y}(s)=\frac{k G(s)}{1+k G(s)},
$$

and

$$
T_{d y}(s)=\frac{1}{1+k G(s)}
$$

respectively. To examine the robustness of the closed-loop system with respect to plant perturbations, define the sensitivity function

$$
S(s) \triangleq \frac{\frac{\delta T_{r y}(s)}{T_{r y}(s)}}{\frac{\delta G(s)}{G(s)}}=\frac{\delta T_{r y}(s)}{\delta G(s)} \frac{G(s)}{T_{r y}(s)}=\frac{1}{1+k G(s)} .
$$

The high gain control scenario is when we allow $k$ to approach an arbitrarily large number, or in the mathematical sense, to let $k$ approach infinity. It is then easy to see that in the presence of high gain control, the feedback control system above asymptotically achieves perfect tracking $\left(T_{r y}(s)=1\right)$, perfect disturbance rejection $\left(T_{d y}(s)=0\right)$, and is insensitive to plant perturbations $(S(s)=0)$.

To illustrate this, consider the following system.

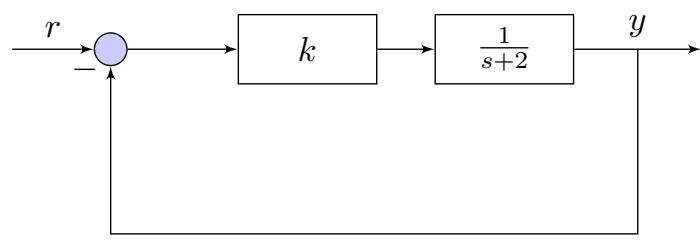

Figure 1.2: An example of high gain control

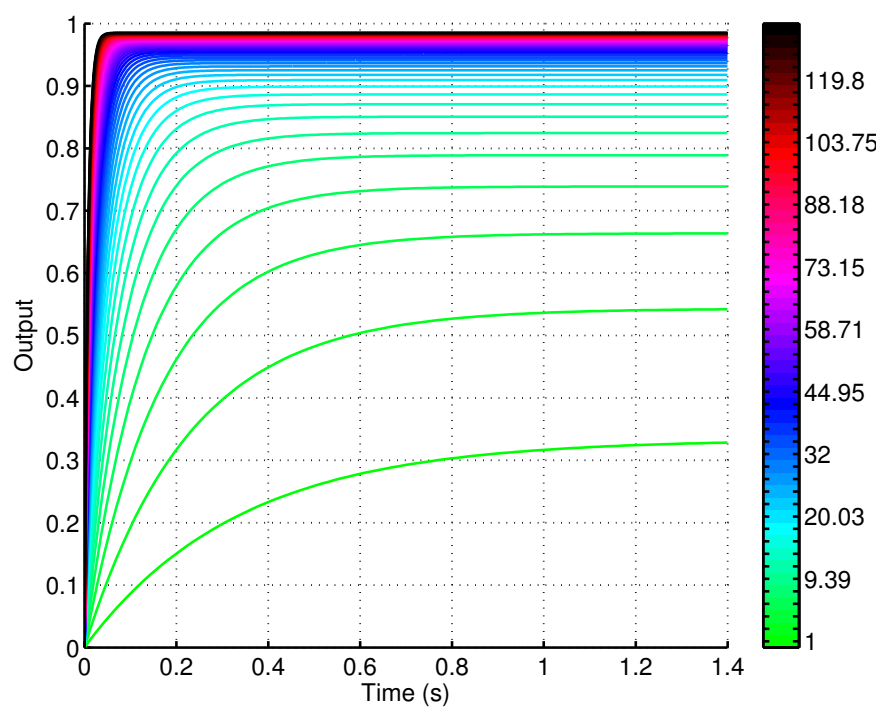

Figure 1.3: Step response of example system as $k$ is increased.

It is easy to verify that the closed-loop system is stable for all $k>-2$. We investigate the closed-loop 
response to a step input. The internal model principle would specify that in the absence of an integrator, a steady-state tracking error in the output would exist which is the case here. However, in light of the above arguments, perfect tracking is attained for arbitrarily large $k$ as seen in Figure 1.3. It may be argued that the simpler solution is to add an integrator instead of applying high gain control. This is indeed true for a step input, but the additional dynamics that the controller would require grows in complexity as the reference trajectory becomes more complicated. The high gain approach works for any reference trajectory.

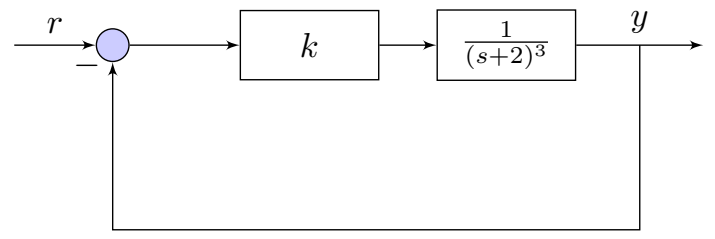

Figure 1.4: An example where high gain control fails

It is important to note that high gain control does not necessarily always produce desirable results. Consider another simple example in Figure 1.4. If $k$ is increased indefinitely, the closed-loop system will become unstable! An application of the standard root locus arguement will reveal that, in the example of Figure 1.4. one of the closed-loop poles will always be in the open right-half plane for sufficiently large $k$. The structural property known as the relative degree of the open loop system is responsible for this behaviour. It is in this way that one cannot simply automatically apply high gain, and expect great performance. The relative degree, and other structural properties are an integral component to understanding when one may construct controllers for which arbitrarily good performance can be achieved with high gain control.

\subsection{Motivation}

As illustrated by the previous section, high gain control can result in high performance control systems. Specifically, if one allows for higher actuation forces, the achievable performance that can be expected usually increases given suitable circumstances.

The motivation for this thesis is to construct a controller for which the notion of high gain control is always possible for a large class of linear time-invariant systems. The ability to obtain high performance in this way will reveal a deeper understanding of the structural properties of the system in relation to its performance. In particular, we wish to attain this behaviour using controllers of low dynamic order. The dynamic order refers to the number of integrators required to realize the controller and is related to its simplicity.

We seek a family of controllers parameterized by a scalar gain so that arbitrarily fast performance can be obtained if this parameter can be made sufficiently large. If there are restrictions on the size of allowable inputs then one may still be able to achieve acceptable performance by tuning the gain parameter within some prescribed range.

Low dynamic order controllers are of immense practical importance. The complexity of the hardware, or the amount of computation necessary to implement the controller is directly related to its order. Common observer-based techniques will have a state size equal to that of the system under control, thus producing a controller of high dimensionality if the order of the system is large. Towards this end, we 
seek to obtain a family of controllers with low dynamic order which can solve the perfect regulation problem with bounded peaking.

\subsection{Literature Review}

The question of achievable performance when the input is unrestricted has been extensively studied in the past, especially throughout the 1970's and 1980's. Formulating the problem in the cheap control setting is a common approach where a quadratic cost criterion is used and the penalty on the input made small. In this formulation, the controller is designed to minimize the following as $\epsilon$ becomes arbitrarily small

$$
J_{\epsilon}=\int_{0}^{\infty} y(t)^{T} Q y(t)+\epsilon u(t)^{T} R u(t) d t
$$

where $Q$, and $R$ satisfy certain positive definiteness conditions, $y(t)$, and $u(t)$ are the output, and input of the system respectively. When the total cost in this formulation can be made arbitrarily close to zero, perfect regulation results. Kwakernaak and Sivan [19] provided necessary and sufficient conditions for perfect regulation under the cheap control framework for square, invertible systems. Their main result is that perfect regulation is attainable if and only if the system is minimum phase.

Additionally, it may occur that some of the state variables involved become unbounded, as the response becomes infinitely fast. To this end, Francis gave a complete solution of the optimal cheap regulator with bounded peaking in [7, 5] which can be seen as a generalization of [19]. They showed that perfect regulation results if and only if the system is right-invertible, and minimum phase. Additionally, a necessary condition for bounded peaking is that the first Markov Parameter of the system be full rank.

A condition that is common between the aformentioned papers is that the system is minimum phase. More recently, it has been shown that the perfect regulation problem is still solvable when this condition is relaxed by allowing the presence of transmission zeros on the imaginary axis 20 . The observer-based output feedback case has also been studied in 2. The authors here derive necessary and sufficient conditions for the solvability of the perfect regulation problem under output feedback by re-formulating the problem as an $H_{2}$ optimization problem where the initial condition to be regulated is seen as an additive impulsive disturbance to the state equation of the system. They essentially recover the same conditions as Francis, but allow transmission zeros on the imaginary axis.

Many researchers have studied high gain systems of this nature from a different perspective using singular perturbation theory (see [32, 28] and references therein). Since any state space representation of a system can be transformed to

$$
\dot{x}(t)=\left[\begin{array}{l}
\dot{x_{1}}(t) \\
\dot{x_{2}}(t)
\end{array}\right]=\left[\begin{array}{ll}
A_{11} & A_{12} \\
A_{21} & A_{22}
\end{array}\right]\left[\begin{array}{l}
x_{1}(t) \\
x_{2}(t)
\end{array}\right]+\left[\begin{array}{l}
0 \\
B
\end{array}\right] u(t)
$$

where $B$ is invertible, an output feedback of the type $u=\frac{1}{\epsilon}\left(K_{1} x_{1}+K_{2} x_{2}\right)$ results in

$$
\begin{aligned}
\dot{x_{1}}(t) & =A_{11} x_{1}+A_{12} x_{2}, \\
\epsilon \dot{x_{2}}(t) & =\left(\epsilon A_{21}+B K_{1}\right) x_{1}+\left(\epsilon A_{22}+B K_{2}\right) x_{2}
\end{aligned}
$$

which is in the standard form of a singularly perturbed differential equation (see [13]). Thus high gain systems can naturally induce a singularily perturbed system. From this point of view, the feedback gain 
matrix is scaled with a high gain parameter, and closely resembles the formulation of the multivariable root locus problem [14] where instead of state feedback, output feedback is used. This connection was made explicit in [29]. Namely, the asymptotic multivariable root locus can be seen as boundary layers, such as (1.7), of various orders. This multiple time scale behaviour is quite typical of singularly perturbed systems.

Perhaps the closest approach to ours is given by Kimura [12, and Zhang [34]. Kimura gave a complete frequency domain characterization of the perfect regulation and bounded peaking problem. Since much of the results in this thesis are frequency domain based, Kimura's results are vital in showing that perfect regulation with bounded peaking is achieved.

Zhang's work in [34] is reflected in this thesis. His primary contribution is the idea that the closedloop system can be approximated in a useful way under high gain. Zhang appeared to have derived much of his inspiration from Martensson [22, but the proofs to his claims were faulty and in some places incomplete. We formalize and extend much of the previous work from Zhang by combining a generalization of a high gain stabilization result from Martensson [22, aspects of multivariable root locus theory, and a complex variable approach to design low order controllers which solve this problem.

\subsection{Thesis Statement \& Contributions}

The aim of this thesis is to construct controllers of low dynamic order which solve the perfect regulation problem with bounded peaking. In doing so, we believe the main contributions of this thesis are:

1. We obtain controllers which solve the perfect regulation problem with bounded peaking that are of low dynamic order when compared to existing observer-based methods, for example [2]. This is shown in Theorem 4.5 by analyzing the infinite zero structure of the system.

2. A lower bound on the dynamic order of the controller is given. Specifically, the lower bound given by Theorem 4.4 is a function of the highest order of infinite zero of the system.

3. A result on high gain stabilization of a class of systems is derived. Theorem 3.1 can be seen as a generalization of the results from Martensson [22].

4. We formalize and extend much of the previous work from Zhang 34. This culminates in Lemma 4.5 .

5. The controller design is extended, under certain conditions, to the decentralized case. This result is summarized by Theorem 4.6

\subsection{Organization}

The remainder of this thesis is organized as follows. Chapter 2 provides the reader with the necessary background material and intuition for the main results of the thesis. In Chapter 3 we generalize the high gain stabilization result in 22 to produce controllers of low order that are necessary for Chapter 4 which shows that the proposed controller solves the perfect regulation problem with bounded peaking. In Chapter 4 we also extend the centralized controller design to the decentralized case where it is shown that, under certain conditions, the decentralized perfect regulation problem with bounded peaking is 
solvable using the same tools developed previously. Case studies which illustrate the key points of the theory are given in Chapter 5 . Concluding remarks are given in Chapter 6

\subsection{Problem Formulation}

Let $\mathbb{R}(s)$ denote the field of rational, linear time-invariant scalar transfer functions, so that $\mathbb{R}(s)^{m \times n}$ is then the set of all $m \times n$ transfer matrices. Denote $\mathbb{C}_{-}, \mathbb{C}_{+}$as the open left and right half plane of $\mathbb{C}$ respectively. Denote also $\mathbb{R}_{++}$as the strictly positive real line, and $\mathbb{N}$ as the natural numbers excluding zero.

Consider the linear time-invariant, stabilizable, and detectable square system

$$
\begin{aligned}
\Sigma: \quad \dot{x}(t) & =A x(t)+B u(t), \\
y(t) & =C x(t) .
\end{aligned}
$$

where $x(t) \in \mathbb{R}^{n}, u(t), y(t) \in \mathbb{R}^{m}$, and $A, B, C$ are constant matrices of appropriate dimensions. Suppose the controller is of the form,

$$
\begin{aligned}
F_{\epsilon}: \quad \dot{\xi}(t) & =M^{\epsilon} \xi(t)+N^{\epsilon} y(t), \\
u(t) & =K^{\epsilon} \xi(t)+L^{\epsilon} y(t)
\end{aligned}
$$

where $\xi(t) \in \mathbb{R}^{n_{c}}$, and $M^{\epsilon}, N^{\epsilon}, K^{\epsilon}, L^{\epsilon}$ are matrices parameterized by a scalar $\epsilon$. Define:

$$
\begin{aligned}
G(s) & =C\left(s I_{n}-A\right)^{-1} B, \\
C(s, \epsilon) & =K^{\epsilon}\left(s I_{n_{c}}-M^{\epsilon}\right)^{-1} N^{\epsilon}+L^{\epsilon}, \\
W_{x}(s) & =C\left(s I_{n}-A\right)^{-1} .
\end{aligned}
$$

With some straightforward manipulation, the output of the closed-loop system given an initial condition $x(0)$ is,

$$
y^{\epsilon}(s)=T_{x}(s, \epsilon) x(0)
$$

where

$$
\begin{aligned}
T_{x}(s, \epsilon) & =T(s, \epsilon) W_{x}(s), \\
T(s, \epsilon) & =\left[I_{m}-G(s) C(s, \epsilon)\right]^{-1} .
\end{aligned}
$$

We say the controller 1.9 achieves perfect regulation with bounded peaking if:

1. The closed-loop system is asymptotically stable,

2. $\sup _{\epsilon_{0} \geq \epsilon>0} \sup _{t \geq 0}\left\|y^{\epsilon}(t)\right\|<\infty$ for some $\epsilon_{0}>0$

3. $\left(\forall x(0) \in \mathbb{R}^{n}\right)(\forall s \in \mathbb{C})$ achieves perfect initial condition response,

$$
\lim _{\epsilon \rightarrow 0} T_{x}(s, \epsilon) x(0)=0
$$


Condition 2 is essentially the same definition for bounded peaking given in [12, and constrains the output to be uniformly bounded in $\epsilon$. As is well known, bounded poles always exhibit bounded peaking, however Condition 3 forces the response to become infinitely fast as $\epsilon$ becomes small. Thus special attention is required.

We assume the following through the remainder of the thesis.

Assumption 1.1. The system (1.8) is minimum phase, i.e. all roots of the zero polynomial in the Smith-McMillan sense have negative real parts.

Remark 1.1. For the SISO case this ensures the numerator polynomial has roots in $C_{-}$. The MIMO case will be defined in Chapter 2.

Assumption 1.2. The transfer matrix $G(s) \in \mathbb{R}(s)^{m \times m}$ is invertible, i.e. det $G(s) \neq 0$.

Under our assumptions a controller of the form (1.9) always exists to solve this problem as demonstrated by [19, 2].

As mentioned previously, the standard approach to solving this problem is to use the Linear Quadratic Regulator theory in the cheap control framework to produce an observer-based controller. By this methodology, the control design is straight forward since the engineer need only solve a Riccati equation. No further information besides its state space representation is required, but the order of the resulting controller will be the same as that of the system. We explore in this thesis that if the designer considers further properties of the system, a potentially significant reduction in the order of the controller can be obtained. Furthermore, we explicitly construct these controllers. 


\section{Chapter 2}

\section{Background}

The methods employed in this thesis are primarily tools from the frequency domain representation of linear time-invariant systems. A brief overview of algebra is given to provide the mathematical foundation for the material from which the standard definitions of poles, and zeros of a transfer matrix are formed. We believe that for pedagogical reasons, this approach is the most complete and intuitive way to present the theory of transfer matrices. The subsequent section reviews some of the basic concepts from the standard SISO root locus problem and details the construction of the multivariable root locus which is essential for the chapters to come. Lastly, we re-state some of the main results from the classical cheap control problem, and its relation to perfect regulation with bounded peaking. Some additional connections to the multivariable root locus are made.

\subsection{Preliminary Algebra}

From a frequency domain perspective, the definition for the poles, and zeros of a MIMO system are intricately related to the structure of the Smith-McMillan form of the corresponding system. Since this is an algebraic notion which may be a somewhat obscure topic to the engineer, we first give an extremely selective overview of algebra. An excellent reference for the material in this section can be found in [1] for the Smith-McMillan form, and its discussion in relation to transfer matrices. For a quick treatment of algebra we refer the reader to the appendix of [30, and for a comprehensive overview [21].

\subsubsection{Rings}

In the mathematical sense, a ring intuitively can be thought of as a generalization of the field of real numbers. The reals restricts us to the set of real numbers but a ring is equipped to handle any set of elements given that they satisfy certain axioms. We provide them here.

Definition 2.1. A ring is a non-empty set $\boldsymbol{R}$ along with the binary operations known as addition (+), and multiplication (·) so that the following are satisfied 
1. $(\boldsymbol{R},+)$ is a commutative group, i.e.

$$
\begin{aligned}
& (\forall a, b, c \in \boldsymbol{R}) a+(b+c)=(a+b)+c, \\
& (\forall a, b \in \boldsymbol{R}) a+b=b+a, \\
& (\exists 0 \in \boldsymbol{R})(\forall a \in \boldsymbol{R}) 0+a=a+0=a, \\
& (\forall a \in \boldsymbol{R})(\exists-a \in \boldsymbol{R}) a+(-a)=0 .
\end{aligned}
$$

2. $(\boldsymbol{R}, \cdot)$ is a semi-group, i.e.

$$
(\forall a, b, c \in \boldsymbol{R}) a \cdot(b \cdot c)=(a \cdot b) \cdot c .
$$

Remark 2.1. It is customary to denote $a+(-b) \triangleq a-b$, and $a \cdot b \triangleq a b$.

Definition 2.2. A commutative ring with identity $\boldsymbol{R}$, is a ring that additionally satisfies

$$
\begin{aligned}
& (\forall a, b \in \boldsymbol{R}) a \cdot b=b \cdot a, \\
& (\exists 1 \in \boldsymbol{R})(\forall a \in \boldsymbol{R}) 1 \cdot a=a \cdot 1=a .
\end{aligned}
$$

The identity element 1 is sometimes denoted the unit element, or unit of a binary relation.

A simple example of a ring is the set of integers. It is also a commutative ring with identity. An example of a ring without identity but is commutative is the set of all even integers (since the set lacks $1)$.

Definition 2.3. A ring $\boldsymbol{R}$ is an integral domain (or domain) if

$$
(\forall a, b \in \boldsymbol{R}) a \cdot b=0 \Rightarrow a=0 \text { or } b=0 .
$$

The set of integers is an integral domain, but the set of $2 \times 2$ matrices over the integers is not.

Definition 2.4. Let $\boldsymbol{R}$ be a commutative ring with identity. An element $x \in \boldsymbol{R}$ is a unit if there exists $a \in \boldsymbol{R}$ such that $a x=x a=1$.

Remark 2.2. Note that unit in the above context refers to "units of a ring", and not "unit element", or "identity" of a binary relation.

Thus the units of a ring are exactly those elements for which there exists an inverse that is also contained in the ring.

Definition 2.5. A field $\boldsymbol{F}$ is a commutative ring with identity satisfying

$$
(\forall a \neq 0 \in \boldsymbol{F}) a \text { is a unit. }
$$

The real, and complex numbers are examples of fields. We wish now to develop the field of fractions associated with a ring so that we may discuss fractions in the usual sense.

Definition 2.6. Let $\boldsymbol{R}$ be a ring. An element $a \in \boldsymbol{R}$ is an absolute non-divisor of zero if

$$
(\forall b \in \boldsymbol{R}) a b=0 \Rightarrow b=0 .
$$


The set of all units of a ring are all absolute non-divisors of zero. In the ring of $n \times n$ matrices over the integers, a matrix is an absolute non-divisor of zero if and only if its determinant is non-zero. This shows that there are other elements besides the units of a ring that can be a non-divisor of zero. If a ring is also a domain, then every non-zero element is an absolute non-divisor of zero.

Definition 2.7. Let $\boldsymbol{R}$ be a ring. A set $\boldsymbol{M} \subset \boldsymbol{R}$ is a multiplicative system if

$$
(\forall a, b \in M) a b \in M \text {. }
$$

Theorem 2.1. The set $\boldsymbol{N}$ of absolute non-divisors of zero in a ring $\boldsymbol{R}$ is a multiplicative system.

It is now possible to construct the field of fractions associated with a ring.

Theorem 2.2. Let $\boldsymbol{R}$ be a ring, $\boldsymbol{M}$ be a multiplicative system in $\boldsymbol{R}$ containing 1 , where $\boldsymbol{M} \subset \boldsymbol{N}$. Define the following equivalence relation $\sim$ on $\boldsymbol{R} \times \boldsymbol{M}$

$$
(\forall(a, b),(c, d) \in \boldsymbol{R} \times \boldsymbol{M})(a, b) \sim(c, d) \Leftrightarrow a d=b c
$$

and denote the set of equivalence classes $\boldsymbol{R} \times \boldsymbol{M} / \sim$ as $\boldsymbol{L} . \boldsymbol{L}$ is a field which contains $\boldsymbol{R}$ as a subring.

The set $\mathbf{L}$ is the field of fractions associated with $\mathbf{R}$ where $(a, b) \in \mathbf{L}$ is simply $\frac{a}{b}$. The equivalence relation above simply states that two fractions $(a, b),(c, d) \in \mathbf{L}$ are equal if $a d=b c$.

Definition 2.8. Let $\boldsymbol{R}$ be a ring. Then $\boldsymbol{I} \subset \boldsymbol{R}$ is an ideal if

$$
\begin{aligned}
& (I,+) \text { is a sub-group of }(R,+), \\
& (\forall a \in \boldsymbol{R})(\forall b \in \boldsymbol{I}) a b \in \boldsymbol{I}, \text { and } b a \in \boldsymbol{I} .
\end{aligned}
$$

The ideal generated by an element $a \in \boldsymbol{R}$ is then the set of all left and right multiples of a.

Definition 2.9. Let $\boldsymbol{R}$ be a ring. An ideal $\boldsymbol{I}$ is called principal if

$$
(\exists a \in \boldsymbol{R}) I=\{a b: b \in \boldsymbol{R}\}
$$

i.e. if it can be generated by a single element.

Every ring always has two principal ideals. They are the ideals generated by 0 , and 1 .

Definition 2.10. A commutative ring with identity $\boldsymbol{R}$ is a principal ideal ring if every ideal in $\boldsymbol{R}$ is principal. $\boldsymbol{R}$ is a principal ideal domain if it is a principal ideal ring and a domain.

Definition 2.11. Let $\boldsymbol{R}$ be a principal ideal domain. Elements $x, y \in \boldsymbol{R}$ are relatively prime if every greatest common denominator of $x, y$ is a unit.

Note that the greatest common denominator by the above definition is unique up to multiplication by a unit of $\mathbf{R}$.

Theorem 2.3. Let $\boldsymbol{R}$ be a principal ideal domain. Elements $x, y \in \boldsymbol{R}$ are relatively prime if and only if there exists $p, q \in \boldsymbol{R}$ such that $p x+q y=1$. 


\subsubsection{Matrix Rings \& Canonical Forms}

We now expand the previous developments to matrices over rings. Let $\mathbf{R}$ be a principal ideal domain, and $\mathbf{R}^{n \times n}$ be the ring of $n \times n$ matrices over $\mathbf{R}$. Also, denote $\mathbf{F}$ as the field of fractions associated with $\mathbf{R}$, and $\mathbf{F}^{n \times n}$ be the ring of $n \times n$ matrices over $\mathbf{F}$. The results follow for the non-square case as well.

Definition 2.12. A matrix $A \in \boldsymbol{R}^{n \times n}$ is unimodular if it is a unit of $\boldsymbol{R}^{n \times n}$.

Theorem 2.4. A matrix $A \in \boldsymbol{R}^{n \times n}$ is unimodular if and only if $\operatorname{det} A$ is a unit of $\boldsymbol{R}$.

Definition 2.13. For $A, B \in \boldsymbol{R}^{n \times n}$, we say that $A$ is equivalent to $B$ if there exists unimodular matrices $P, Q \in \boldsymbol{R}^{n \times n}$ such that $A=P B Q$.

If $\mathbf{R}=\mathbb{R}$ then every $A \in \mathbb{R}^{n \times n}$ is equivalent to a diagonal matrix $\Sigma$ containing the singular values of $A$.

We now give the important canonical forms associated with $\mathbf{R}^{n \times n}$, and $\mathbf{F}^{n \times n}$. The next result states that every $A \in \mathbf{R}^{n \times n}$ is equivalent to a diagonal matrix known as the Smith Normal Form.

Theorem 2.5. Every matrix $A \in \boldsymbol{R}^{n \times n}$ of rank $r$ is equivalent to a diagonal matrix of the form

$$
D(A) \triangleq\left[\begin{array}{cccc}
\epsilon_{1} & 0 & \cdots & 0 \\
0 & \ddots & & \vdots \\
\vdots & & \epsilon_{r} & 0 \\
0 & \cdots & 0 & 0
\end{array}\right]
$$

known as the Smith Normal Form. In addition, $\epsilon_{i}$ divides $\epsilon_{i+1}$ for $i=1, \ldots, r-1$. The $\left\{\epsilon_{i}\right\}$ are the invariant factors of $A$.

A similar canonical form for $\mathbf{F}^{n \times n}$ can be given and is known as the Smith-McMillan Form. It is a direct consequence of factoring out the lowest common multiple of all denominators $\Psi$ of $A \in \mathbf{F}^{n \times n}$ to give $A=\frac{1}{\Psi} B$ where now $B$ belongs to $\mathbf{R}^{n \times n}$ to which the Smith Normal Form applies.

Theorem 2.6. Every matrix $A \in \boldsymbol{F}^{n \times n}$ of rank $r$ is equivalent to a diagonal matrix of the form

$$
D_{M}(A) \triangleq\left[\begin{array}{cccc}
\frac{\epsilon_{1}}{\psi_{1}} & 0 & \cdots & 0 \\
0 & \ddots & & \vdots \\
\vdots & & \frac{\epsilon_{r}}{\psi_{r}} & 0 \\
0 & \cdots & 0 & 0
\end{array}\right]
$$

known as the Smith-McMillan Form. In addition, $\epsilon_{i}$ divides $\epsilon_{i+1}$, and $\psi_{i+1}$ divides $\psi_{i}$ for $i=1, \ldots, r-1$. Denote

$$
\begin{aligned}
& \Psi=\prod_{i=1}^{r} \psi_{i}, \\
& \Phi=\prod_{i=1}^{r} \epsilon_{i}
\end{aligned}
$$

as the denominator, and numerator factors in the Smith-McMillan sense. 


\subsubsection{The Ring of Transfer Matrices \& Infinite Zeros}

It is well known that the set of all polynomials in the indeterminate $s$ and coefficients in a field $\mathbf{F}$ forms a principal ideal domain, $\mathbf{R}[s]$. If we make the distinction that $\mathbf{F}=\mathbb{R}$ the real numbers, we denote $\mathbb{R}[s] \triangleq \mathbf{R}[s]$. Denote the field of fractions associated with $\mathbb{R}[s]$ as $\mathbb{R}(s)$. $\mathbb{R}(s)$ is then the field of all rational functions in $s$, or in our context the field of all rational transfer functions. $\mathbb{R}(s)^{m \times m}$ is then the ring of all $m \times m$ transfer matrices.

We thus have the following standard definitions for the poles and zeros of a transfer matrix.

Definition 2.14. The poles and zeros of a transfer matrix $A \in \mathbb{R}(s)^{m \times m}$ of rank $r$ are respectively the roots of the denominator, and numerator factors, i.e.

$$
\begin{aligned}
& \Psi=\prod_{i=1}^{r} \psi_{i} \\
& \Phi=\prod_{i=1}^{r} \epsilon_{i}
\end{aligned}
$$

associated with $D_{M}(A)$. Denote $\Psi(\Phi)$ as the pole (zero) polynomial in the Smith-McMillan sense.

Remark 2.3. With the above definition of the zeros of a transfer matrix, a system is minimum phase if the roots of its zero polynomial in the Smith-McMillan sense are contained in the open left-half plane. In the SISO case, this specializes to the roots of its numerator polynomial as expected.

With the above definition we can then appropriately define infinite zeros which will become central to this thesis in later chapters.

Definition 2.15. A transfer matrix $G(s)$ has an infinite zero of order $\sigma$ if $G\left(\frac{1}{s}\right)$ has a finite zero of order $\sigma$ at $s=0$.

As a simple illustration, consider the following example. Note that the notion of finite and infinite zeros of a system are integral in understanding the minimum phase assumption and the controller design methodology respectively.

Example 2.1. Let

$$
G(s)=\left[\begin{array}{cc}
0 & \frac{1}{s+1} \\
\frac{s+1}{(s+3)^{3}} & 0
\end{array}\right]=\frac{1}{(s+1)(s+3)^{3}}\left[\begin{array}{cc}
0 & (s+3)^{3} \\
(s+1)^{2} & 0
\end{array}\right]
$$

The Smith Normal form of the above polynomial matrix is given by

$$
D\left(\left[\begin{array}{cc}
0 & (s+3)^{3} \\
(s+1)^{2} & 0
\end{array}\right]\right)=\left[\begin{array}{cc}
1 & 0 \\
0 & (s+3)^{3}(s+1)^{2}
\end{array}\right]
$$

so that the Smith-McMillan form of $G(s)$ is

$$
D_{M}(G(s))=\left[\begin{array}{cc}
\frac{1}{(s+1)(s+3)^{3}} & 0 \\
0 & s+1
\end{array}\right]
$$

Note that the entries in the Smith-McMillan form need not be proper. The poles of $G(s)$ are then the 
roots of $(s+1)(s+3)^{3} \cdot 1$, and similarly the zeros are the roots of $1 \cdot(s+1)$. This also illustrates that MIMO systems can have poles and zeros at the same location in the complex plane without cancellation.

To calculate the infinite zeros of $G(s)$, let $z=\frac{1}{s}$ so that the Smith-McMillan form of $G(z)$ is

$$
\begin{aligned}
D_{M}(G(z)) & =D_{M}\left(\left[\begin{array}{cc}
0 & \frac{z}{z+1} \\
\frac{z^{2}(z+1)}{(3 z+1)^{3}} & 0
\end{array}\right]\right) \\
& =\frac{1}{(3 z+1)^{3}(z+1)} D\left(\left[\begin{array}{cc}
0 & z(3 z+1)^{3} \\
z^{2}(z+1)^{2} & 0
\end{array}\right]\right) \\
& =\frac{1}{(3 z+1)^{3}(z+1)}\left[\begin{array}{cc}
z & 0 \\
0 & z^{2}(z+1)^{2}(3 z+1)^{3}
\end{array}\right] \\
& =\left[\begin{array}{cc}
z \frac{1}{(3 z+1)^{3}(z+1)} & 0 \\
0 & z^{2}(z+1)
\end{array}\right] .
\end{aligned}
$$

Thus, $G(s)$ has first, and second orders of infinite zeros corresponding to the $z$ and $z^{2}$ terms. It is incorrect to simply substitute $z=\frac{1}{s}$ into $D_{M}(G(s))$ since the unimodular matrices which take $G(s)$ into its Smith-McMillan form eliminate the information on the infinite zeros. For details on why this occurs see [11]. Notice that there is an ordering to the $z$ terms in $D_{M}(G(z))$. This ordering is present as the numerator of the $i^{\text {th }}$ diagonal term in the Smith-McMillan form must divide the numerator in the $(i+1)^{\text {th }}$ diagonal term.

We note that although the Smith-McMillan form is an adequate theoretical tool, the poles and zeros are typically not calculated in this way numerically. Determining the Smith Normal form is non-trivial by hand. In the next section, an algorithm for determining the infinite zeros from the multivariable root locus literature is given that is easier to use.

\subsection{Multivariable Root Locus}

As much of the compensator design relies on the inherent properties of the multivariable root locus we wish to provide a brief exposition on the main results of the root locus problem. The necessary intuition that is required in later chapters is presented here.

\subsubsection{The Standard SISO Root Locus Problem}

Every undergraduate who has taken an introductory classical control course will have seen the development of the root locus technique for single-input single-ouput systems. The basic question that the standard root locus problem seeks to answer is: what happens to the closed-loop behaviour as the open loop gain parameter varies from $0 \rightarrow \infty$ ?

As is well known, since the closed-loop behaviour is characterized by the closed-loop poles, we only state and remind the reader of the standard results commonly used in graphically tracing the various root loci. A light treatment of the SISO root locus can be found in [4, and the more traditional overview in [17. For a rigorous and complete version of the developments we refer the reader to [16.

Consider Figure 2.1 where $G(s)$ is a SISO system of relative degree $r=n-m>0$. We summarize a subset of the main facts below: 


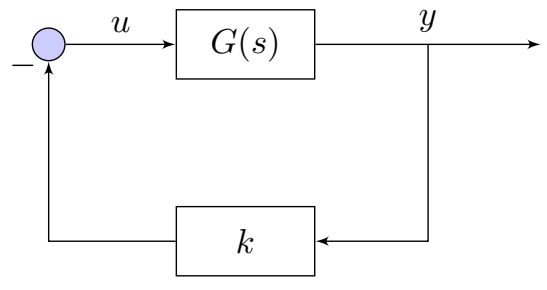

Figure 2.1: The SISO root locus setup

1. The root loci begin $(k=0)$ at the $n$ open loop poles of $G(s)$.

2. The root loci terminate $(k=\infty)$ at the $n$ open loop zeros of $G(s)$. $m$ root loci terminate at the finite open loop zeros, and the $r$ remaining loci terminate at the point of infinity on the extended complex plane $(\mathbb{C} \cup \infty)$. $G(s)$ is said to have $r$ infinite zeros since $G(\infty)=0$.

3. The $r$ root loci which approach infinity do so according to the following theorem.

Theorem 2.7. [16] The $r=n-m$ root loci which approach infinity as $k$ approaches infinity do so according to

$$
s_{j}=\frac{\sum_{i=1}^{n} p_{i}-\sum_{i=1}^{m} z_{i}}{n-m}+k^{\frac{1}{n-m}} e^{i \frac{\theta+2 \pi j}{n-m}}
$$

for $j=0, \ldots, n-m-1$, where $p_{i}, z_{i}$ are the finite open loop poles and zeros respectively. Additionally, $s_{j}$ becomes an arbitrarily good estimate for the $j^{\text {th }}$ root loci.

4. The root locus is symmetric about the real axis.

Definition 2.16. [25] A Butterworth pattern of order $\sigma \in \mathbb{N}$ is a cyclic arrangement of asymptotes of a common intersection point where the angles between the asymptotes are equal to $\frac{2 \pi}{\sigma}$.

Remark 2.4. In Theorem 2.7 a SISO system of relative degree $r$ will have unbounded root loci that form a Butterworth pattern of order $r$.

Remark 2.5. As can be inferred from Theorem 2.7, if $r>2$ then necessarily there will be an asymptote pointing into the right half plane of $\mathbb{C}$ resulting in a unstable closed-loop system for sufficiently large $k$.

There is a plethora of other facts which dictate angles of approach/departure and break-in/away points of the root locus but are less significant to understand from the point of view of the results in this thesis. Thus, we shall omit them.

\subsubsection{Multivariable Generalizations}

The situation when $G(s) \in \mathbb{R}(s)^{m \times m}$ is far more complicated. We follow the developments of [14] closely and explore the asymptotic behaviour of the multivariable root locus first. It will be shown that the unbounded closed-loop poles approach infinity in several Butterworth patterns whose orders, under certain assumptions, are equal to the orders of infinite zeros of the system. As expected, the SISO version has only a single order of infinite zeros so that there is only one Butterworth pattern as seen by Theorem 2.7 .

Suppose

$$
G(s)=C\left(s I_{n}-A\right)^{-1} B \in \mathbb{R}(s)^{m \times m}
$$




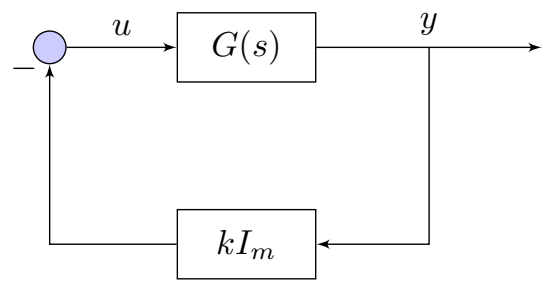

Figure 2.2: The MIMO root locus setup

is a $m \times m$ transfer matrix and consider Figure 2.2 .

The closed-loop poles are given by the roots of the following equation

$$
\Delta(s, k) \triangleq \operatorname{det}\left(I_{m}+k G(s)\right)=0
$$

where the matrix $I_{m}+k G(s)$ is commonly known as the return-difference operator. Suppose we allow $g=-\frac{1}{k}$ to be a complex number. Then

$$
\Delta(s, g)=\operatorname{det}\left(g I_{m}-G(s)\right)=0 .
$$

$\Delta(s, g)$ describes an eigvenvalue equation of two complex variables $s$, and $g$. Clearly the $m$ eigenvalues of $G(s), g$, are a function of $s$.

In the multivariable root locus literature $g(s)$ is known as the characteristic gain function, and is an algebraic function [25. Unlike the SISO case, where the domain of the characteristic gain function is a single copy of the complex plane, here the domain of $g(s)$ is a Riemann surface formed by appropriately stitching $m$ copies of the complex plane together. The construction of such a Riemann surface is given in [25] and its discussion is beyond the scope of this thesis. The entirety of the multivariable root locus can be derived from the algebraic function point of view, but we do not explore any further in this direction.

Instead, we return to 2.16) since the algorithms developed from this point of view will be used to develop controllers in Chapter 4 . We will revisit the algebraic function approach in a different context in Chapter 3. Write the Taylor series expansion of $G_{1}(s) \triangleq G(s)$ around the point at infinity to obtain

$$
\begin{aligned}
G_{1}(s) & =\sum_{i=1}^{\infty} \frac{1}{s^{i}} C_{1} A_{1}^{i-1} B_{1} \\
& =\frac{1}{s} C_{1} B_{1}+\sum_{i=2}^{\infty} \frac{1}{s^{i}} C_{1} A_{1}^{i-1} B_{1} \\
& \triangleq \frac{1}{s} G_{1}+P_{1}(s) .
\end{aligned}
$$

Definition 2.17. Denote $G_{1}$ as the first Projected Markov Parameter (PMP).

As we shall see, $G_{1}$ or the first PMP plays a large role in determining the behaviour of the first order root loci, and the first order infinite zeros. Let the eigenvalue decomposition of $G_{1}$ be

$$
G_{1}=\left[\begin{array}{ll}
U_{1} & M_{1}
\end{array}\right]\left[\begin{array}{cc}
\Lambda_{1} & 0 \\
0 & 0_{d_{1}}
\end{array}\right]\left[\begin{array}{l}
V_{1} \\
N_{1}
\end{array}\right]
$$

where $\Lambda_{1}$ is the Jordan form of $G_{1}, 0_{d_{1}}$ is a square zero matrix of size $d_{1} \geq 0$, and $U_{1}, M_{1}, V_{1}, N_{1}$ 
are constant matrices of appropriate dimensions. The column and row vectors of $U_{1}$, and $V_{1}$ are the associated eigenvectors and dual eigenvectors respectively. $M_{1}, N_{1}$ are then matrices containing the eigenvectors of the 0 eigenvalues.

Definition 2.18. $G_{1}$ is said to satisfy Simple Null Structure (SNS) if the Jordan canonical blocks associated with the 0 eigenvalue appear in blocks of size 1 . If there are no 0 eigenvalues then SNS is trivially satisfied.

The case when $G_{1}$ does not satisfy SNS is mathematically involved and is omitted for simplicity. Its implications will be explored somewhat briefly later.

Suppose there is a non-zero number of 0 eigenvalues in $G_{1}$, i.e. $d_{1}>0$. If we substitute 2.20 into 2.16) we obtain

$$
\begin{aligned}
\Delta_{1}(s, k) & =\operatorname{det}\left(I_{m}+k G_{1}(s)\right) \\
& =\operatorname{det}\left(I_{m}+\frac{k}{s} G_{1}+k P_{1}(s)\right) \\
& =\operatorname{det}\left(\left[\begin{array}{cc}
I_{m-d_{1}}+\frac{k}{s} \Lambda_{1}+k V_{1} P_{1}(s) U_{1} & k V_{1} P_{1}(s) M_{1} \\
k N_{1} P_{1}(s) U_{1} & I_{d_{1}}+k N_{1} P_{1}(s) M_{1}
\end{array}\right]\right) \\
& =0 .
\end{aligned}
$$

An application of Schur's formula for determinants gives

$$
\Delta_{1}(s, k) \triangleq \Delta_{1}^{1}(s, k) \Delta_{1}^{2}(s, k)
$$

where

$$
\begin{aligned}
& \Delta_{1}^{1}(s, k)=\operatorname{det}\left(I_{m-d_{1}}+\frac{k}{s} \Lambda_{1}+k V_{1} P_{1}(s) U_{1}\right) \\
& \Delta_{1}^{2}(s, k)=\operatorname{det}\left(I_{d_{1}}+k N_{1} P_{1}(s) M_{1}-k^{2} N_{1} P_{1}(s) U_{1}\left(I_{m-d_{1}}+\frac{k}{s} \Lambda_{1}+k V_{1} P_{1}(s) U_{1}\right)^{-1} V_{1} P_{1}(s) M_{1}\right) .
\end{aligned}
$$

The roots of 2.27) describes all closed-loop poles which vanish to the first order of infinite zeros according to the relationship

$$
s=\lambda k .
$$

On substitution of 2.29) into 2.27) and solving for $\lambda$, Kouvaritakis \& Shaked [15] give the following result.

Theorem 2.8. [15] For $G_{1}(s) \in \mathbb{R}(s)^{m \times m}$, if the first $P M P G_{1}$ satisfies $S N S$ and has $d_{1}$ zero eigenvalues, $G_{1}(s)$ has $m-d_{1}$ first order infinite zeros. The angles of the asymptotes are given by the phase of each non-zero eigenvalue of $G_{1}$.

The relationship of all closed-loop poles associated with orders of infinite zeros higher than 1 are given by 2.28. If we consider the second order law

$$
s^{2}=\lambda k
$$


it can be shown that 2.28 reduces to

$$
\begin{aligned}
\Delta_{1}^{2}(s, k) & =\operatorname{det}\left(I_{d_{1}}+k N_{1} P_{1}(s)\left(I_{m}+s U_{1} \Lambda_{1}^{-1} V_{1} P_{1}(s)\right)^{-1} M_{1}\right) \\
& \triangleq \operatorname{det}\left(I_{d_{1}}+k G_{2}(s)\right)
\end{aligned}
$$

with $G_{2}(s)$ characterized by the following lemmas.

Lemma 2.1. 14 $G_{2}(s)=C_{2}\left(s I-A_{2}\right)^{-1} B_{2}$ where

$$
\begin{aligned}
& A_{2}=\left(I-B_{1} U_{1} \Lambda_{1}^{-1} V_{1} C_{1}\right) A_{1}, \\
& B_{2}=B_{1} M_{1}, \\
& C_{2}=N_{1} C_{1} .
\end{aligned}
$$

Lemma 2.2. [14] Suppose $G_{1}(s)$ has orders of infinite zeros equal to $\left\{\sigma_{1}, \sigma_{2}, \ldots, \sigma_{v}\right\} . G_{2}(s)$ as defined above has orders of infinite zeros equal to $\left\{\sigma_{2}, \ldots, \sigma_{v}\right\}$.

Note that in the Taylor series expansion around infinity for $G_{2}(s)$

$$
\begin{aligned}
G_{2}(s) & =\sum_{i=1}^{\infty} \frac{1}{s^{i}} C_{2} A_{2}^{i-1} B_{2} \\
& =C_{2} A_{2} B_{2}+\sum_{i=3}^{\infty} \frac{1}{s^{i}} C_{2} A_{2}^{i-1} B_{2} \\
& \triangleq \frac{1}{s^{2}} G_{2}+P_{2}(s)
\end{aligned}
$$

the first Markov Parameter $C_{2} B_{2}$ is identically zero since by Lemma 2.2, $G_{2}(s)$ has no first order infinite zeros.

Definition 2.19. Denote $G_{2}$ as the second Projected Markov Parameter (PMP).

The reader will note that we are now in the same situation as 2.20 where instead of $G_{1}(s)$, we have $G_{2}(s)$ that has all but the first orders infinite zeros. If we recursively follow the procedure outlined above, the asymptotic root locus can be summarized in the following two theorems.

Theorem 2.9. 14] The system $G_{i+1}(s)$ is equal to

$$
G_{i+1}(s)=C_{i+1}\left(s I-A_{i+1}\right)^{-1} B_{i+1}
$$

where

$$
\begin{aligned}
& A_{i+1}=\left(I-B_{i} U_{i} \Lambda_{i}^{-1} V_{i} C_{i} A_{i}^{i-1}\right) A_{i}, \\
& B_{i+1}=B_{i} M_{i}, \\
& C_{i+1}=N_{i} C_{i},
\end{aligned}
$$

and the $(i+1)^{\text {th }}$ PMP is

$$
G_{i+1}=C_{i+1} A_{i+1}^{i} B_{i+1}
$$

Definition 2.20. A system $G(s)$ is said to satisfy SNS if all its PMPs satisfy SNS. 
Remark 2.6. Byrnes 8 Stevens [1] have shown that SNS is necessary and sufficient for the orders of the Butterworth patterns in the asymptotic root locus to coincide with the orders of infinite zeros. In addition, SNS is a generic property.

Theorem 2.10. The asymptotes to which the closed-loop poles of $G(s)$ approach infinity appear in Butterworth patterns according to the laws

$$
s=\left(-\lambda_{j}^{(i)} k\right)^{\frac{1}{i}}
$$

and form angles with the positive real axis given by

$$
\alpha_{j}^{(i)}=\frac{1}{i}\left(\arg \left(-\lambda_{j}^{(i)}\right)+2 \pi t\right)
$$

where $i=1, \ldots, v, j=1, \ldots, d_{i-1}-d_{i}, t=0, \ldots, i-1, \lambda_{j}^{(i)}$ is the $j^{\text {th }}$ non-zero eigenvalue of the $i^{\text {th }}$ $P M P$, and $d_{i}$ is equal to the number of zero eigenvalues of $i^{\text {th }} P M P\left(d_{0} \triangleq m\right)$. Additionally, there are

$$
n_{i}=i\left(d_{i-1}-d_{i}\right)
$$

$i^{\text {th }}$ order infinite zeros.

Remark 2.7. As can be seen from the above theorem, the PMPs of a system determine the behaviour of the multivariable root locus.

As will become apparent in later chapters, we are particularly interested in the asymptotic behaviour of the closed-loop poles. Under the SNS assumption for each PMP, we have seen that the asymptotic behaviour is dictated only by the orders of infinite zeros.

To illustrate, consider the following example.

Example 2.2. Let

$$
G(s)=\left[\begin{array}{cc}
\frac{1}{s+1} & 0 \\
0 & \frac{s+1}{(s+3)^{3}}
\end{array}\right] .
$$

Since the system is diagonal, we would expect the MIMO root locus to be just the root locus of each individual SISO system. We will use the above theory to demonstrate that this is indeed true. A state space realization of $G(s)$ is

$$
\begin{aligned}
& \dot{x}(t)=\left[\begin{array}{cccc}
-1 & 0 & 0 & 0 \\
0 & -9 & -3.375 & -1.6875 \\
0 & 8 & 0 & 0 \\
0 & 0 & 2 & 0
\end{array}\right] x(t)+\left[\begin{array}{cc}
1 & 0 \\
0 & 0.5 \\
0 & 0 \\
0 & 0
\end{array}\right] u(t), \\
& y(t)=\left[\begin{array}{cccc}
1 & 0 & 0 & 0 \\
0 & 0 & 0.25 & 0.125
\end{array}\right] x(t)
\end{aligned}
$$


so that the eigenvalue decomposition of the first PMP is

$$
\begin{aligned}
G_{1}=C B \triangleq C_{1} B_{1}=\left[\begin{array}{ll}
1 & 0 \\
0 & 0
\end{array}\right] & =\left[\begin{array}{ll}
U_{1} & M_{1}
\end{array}\right]\left[\begin{array}{cc}
\Lambda_{1} & 0 \\
0 & 0_{d_{1}}
\end{array}\right]\left[\begin{array}{l}
V_{1} \\
N_{1}
\end{array}\right] \\
& =\left[\begin{array}{ll}
1 & 0 \\
0 & 1
\end{array}\right]\left[\begin{array}{ll}
1 & 0 \\
0 & 0
\end{array}\right]\left[\begin{array}{ll}
1 & 0 \\
0 & 1
\end{array}\right] .
\end{aligned}
$$

Since the rank defect of $G_{1}$ is $d_{1}=1$ we have $1 \cdot\left(d_{0}-d_{1}\right)=1$ first order infinite zeros. Let us now compute the second PMP. By Theorem 2.9.

$$
\begin{aligned}
G_{2} & =C_{2} A_{2} B_{2} \\
& =N_{1} C_{1}\left(I_{4}-B_{1} U_{1} \Lambda_{1}^{-1} V_{1} C_{1} A_{1}^{0}\right) A_{1} B_{1} M_{1} \\
& =1 .
\end{aligned}
$$

As $G_{2}$ is non-singular, we terminate the process. Therefore, the system has $2 \cdot\left(d_{1}-d_{2}\right)=2$ second order infinite zeros, and a single first order infinite zero. According to Theorem 2.10, the angle of the asymptote with respect to the positive real axis associated with the first order infinite zero is

$$
\alpha_{1}^{(1)}=\pi
$$

and for the second order infinite zeros are

$$
\alpha_{1}^{(2)}=\left\{\frac{\pi}{2},-\frac{\pi}{2}\right\}
$$

We also note here that the closed-loop poles which remain finite as $k \rightarrow \infty$ tend to those points $s$ for which $G(s)$ loses rank, i.e. the transmission zeros of $G(s)$. In addition, the pivots of the multivariable root locus (or the intersection point of the asymptotes) need not be on the real line as in the SISO case. Symmetry about the real axis is also maintained.

\subsection{Cheap Control \& Perfect Regulation with Bounded Peaking}

For the purposes of generating sufficient intuition for the methodologies used in this thesis, we now provide an overview of the classical cheap control problem and its relation to the perfect regulation problem with bounded peaking. We also make the connection with the multivariable root locus. Much of the material here is borrowed from [19, 7, [5, 12].

The cheap control problem is typically formulated in a linear quadratic setting. Suppose we have a stabilizable, and detectable system

$$
\begin{aligned}
& \dot{x}(t)=A x(t)+B u(t), \\
& y(t)=C x(t)
\end{aligned}
$$

where $x(t), u(t)$, and $y(t)$ are real $n, m$, and $m$ dimensional vectors respectively.

The objective is to determine an input $u(\cdot)$ belonging to the class of signals $\mathcal{U}$ which minimizes the 
cost functional

$$
\min _{u \in \mathcal{U}} J_{\epsilon}=\min _{u \in \mathcal{U}} \int_{0}^{\infty}\|y(t)\|^{2}+\epsilon\|u(t)\|^{2} d t
$$

for a fixed positive scalar $\epsilon . \mathcal{U}$ is defined to ensure that $u$ satisfies certain regularity conditions such as continuity. Suppose that the optimal solution is $u_{\epsilon}^{*}$, and denote the optimal value as $J_{\epsilon}^{*}$. The subscript denotes the quantities dependence on $\epsilon$.

The well-known solution to the cheap control problem is

$$
u_{\epsilon}^{*}=-\frac{1}{\epsilon} B^{T} P_{\epsilon} x
$$

where $P_{\epsilon}$ is the unique solution to the algebraic Riccati equation

$$
A^{T} P_{\epsilon}+P_{\epsilon} A+C^{T} C=\frac{1}{\epsilon} P_{\epsilon} B^{T} B P_{\epsilon}
$$

and the achieved optimal value is

$$
J_{\epsilon}^{*}=x(0)^{T} P_{\epsilon} x(0) .
$$

Of course, the above are all well defined for all non-zero $\epsilon$. The efforts of numerous researchers are aimed at describing the limiting behaviour of the state/input trajectories, and optimal value as $\epsilon \downarrow 0$.

\subsubsection{Perfect Regulation}

When $\epsilon$ is made arbitrarily small, there exists a subspace of the state space denoted as the singular hyperplane. The state trajectory $x_{\epsilon}(t)$ induced by the optimal input $u_{\epsilon}^{*} 2.60$ as $\epsilon \downarrow 0$ converges in the sense of a distribution, or generalized function (see [6]). For all initial conditions $x(0), x(0+)$ lies on this singular hyperplane and evolves forward in time on it. Thus $x(t)$ is discontinuous at $t=0$ and is either a step, impulse, or some higher order singularity. This also implies that the optimal input is discontinuous at $t=0$. Naturally, we can then ask when the following limit exists

$$
\lim _{\epsilon \downarrow 0} J_{\epsilon}^{*} \triangleq J_{0}^{*}=x(0)^{T} P_{0} x(0)
$$

Indeed, Kwakernaak \& Sivan [19] have shown that the above limit exists which can be seen by the following argument. $J_{\epsilon}^{*}$ is certainly bounded below by 0 due to the positive definiteness of the cost functional. Since with decreasing $\epsilon, J_{\epsilon}^{*}$ is non-increasing so that by monotone convergence, $J_{0}^{*}$ must exist, which implies that $P_{0}$ must also exist.

Perfect regulation refers to the situation when it is possible to reduce $J_{\epsilon}^{*}$ to zero as $\epsilon \downarrow 0$, i.e.

$$
J_{0}^{*}=0
$$

Qualitatively, perfect regulation is the instantaneous regulation of the output to zero. For square systems, the main result is the following.

Theorem 2.11. [5] For all $x(0)$, as $\epsilon \downarrow 0$ the optimal value $J_{0}^{*}=0$ if and only if the system is rightinvertible, and minimum phase.

As can be seen from [18], these conditions are quite natural since the input $u_{\epsilon}^{*}$ tries to invert the plant asymptotically as $\epsilon \downarrow 0$. Non-minimum phase plants would then have unstable poles from the process of 
inversion.

\subsubsection{Bounded Peaking}

As the state vector in perfect regulation is being brought to zero arbitrarily fast it is of interest to determine when the resulting trajectory remains bounded in the $\mathcal{L}^{2}$, and $\mathcal{L}^{\infty}$ sense. This is the primary investigation of Francis \& Glover [7.

Suppose that

$$
u_{\epsilon}^{*}=F_{\epsilon} x
$$

so that the closed-loop initial condition response is

$$
x(t)=e^{\left(A+B F_{\epsilon}\right) t} x(0) \triangleq T_{\epsilon}(t) x(0) .
$$

By $\mathcal{L}^{2}$-bounded peaking we refer to $x(t)$ belonging to the class of square integrable functions, or equivalently in light of 2.66 that

$$
\sup \left\{\left\|T_{\epsilon}(t)\right\|_{2}: 0<\epsilon \leq \epsilon_{0}\right\}<\infty
$$

for some $\epsilon_{0}>0$. $\mathcal{L}^{\infty}$-bounded peaking follows an identical definition where the $\infty$ norm is used instead.

We restate the main results from [7].

Theorem 2.12. As $\epsilon \downarrow 0$, the state trajectory $x(t)$ has $\mathcal{L}^{2}$-bounded peaking if and only if the system is minimum phase and

$$
\operatorname{rank} G(s)=\operatorname{rank} C B \text {. }
$$

Theorem 2.13. As $\epsilon \downarrow 0$, the state trajectory $x(t)$ has $\mathcal{L}^{\infty}$-bounded peaking if and only if

$$
\operatorname{rank} G(s)=\operatorname{rank} C B
$$

and the system has zeros in the closed left half plane with only simple zeros on the imaginary axis.

It is important to note that the condition that is common in both Theorem 2.12, and 2.13 is that the first Markov Parameter of $G(s)$, i.e. $G_{1}=C B$, is equal to the rank of $G(s)$. For invertible $G(s)$, as is such in this thesis, this implies that $G_{1}$ is non-singular implying by the previous discussion on the multivariable root locus that the system has only first order infinite zeros, or equivalently that the system has the maximal number of finite zeros. In general, $G(s)$ has several different orders of infinite zeros so that the requirement of the state trajectory $x(t)$ to have bounded peaking in either the $\mathcal{L}^{2}$, or $\mathcal{L}^{\infty}$ sense is quite restrictive.

In this thesis, we are concerned with $\mathcal{L}^{2}$-bounded peaking and any further reference to bounded peaking will mean as such.

\subsubsection{A Characterization of Perfect Regulation with Bounded Peaking}

Since much of the approach to designing controllers in later chapters relies on a complex variable, or frequency domain characterization of the closed-loop poles, we provide a brief account of such a characterization for the perfect regulation problem with bounded peaking. The result here is due to Kimura [12]. 
The output trajectory $y_{\epsilon}(t)$ for a given initial condition $x(0)$ is given by

$$
y_{\epsilon}(t)=C e^{\left(A+B F_{\epsilon}\right) t} x(0) \triangleq X_{\epsilon}(t) x(0)
$$

where $F_{\epsilon}$ is some state feedback with a continuous dependence on a scalar $\epsilon$.

Definition 2.21. The system achieves perfect regulation if $\lim _{\epsilon \downarrow 0} y_{\epsilon}(t)=0$ or equivalently if

$$
\lim _{\epsilon \downarrow 0} \int_{0}^{\infty}\left\|X_{\epsilon}(t)\right\|_{2}^{2} d t=0 .
$$

Definition 2.22. The system achieves bounded peaking if for some $\epsilon_{0}>0$

$$
\sup _{\epsilon \leq \epsilon_{0}} \sup _{t \geq 0}\left\|X_{\epsilon}(t)\right\|<\infty .
$$

Remark 2.8. Here (and throughout the thesis), by bounded peaking, we are referring to the uniform boundedness of the output $y_{\epsilon}(t)$ with respect to $\epsilon$ as given in the problem formulation of the previous chapter, and not boundedness of the state trajectory as detailed in the previous section.

We then have the following frequency domain characterization of the perfect regulation problem with bounded peaking.

Theorem 2.14. Denote $T_{\epsilon}(s)$ as the Laplace transform of $X_{\epsilon}(t)$. The system achieves perfect regulation if and only if for all $s \in \mathbb{C}$

$$
T_{\epsilon}(s) \rightarrow 0, \quad T_{\epsilon}\left(\frac{s}{\epsilon}\right) \rightarrow 0
$$

as $\epsilon \downarrow 0$.

Theorem 2.15. Denote $T_{\epsilon}(s)$ as the Laplace transform of $X_{\epsilon}(t)$. The system achieves bounded peaking if and only if for all $s \in \mathbb{C}$

$$
T_{\epsilon}(s) \rightarrow T_{0}(s), \quad T_{\epsilon}\left(\frac{s}{\epsilon}\right) \rightarrow 0
$$

as $\epsilon \downarrow 0 . T_{0}(s)$ is a strictly proper matrix independent of $\epsilon$.

From the above, perfect regulation always implies bounded peaking of the output. Bounded peaking of the output is easier to attain than bounded peaking of the state trajectory due to the restriction on the orders of infinite zeros which are a structural property of the system.

We can now conclude that the perfect regulation problem with bounded peaking can be solved if it can be shown that the transfer matrix multiplying the initial condition can be shown to converge to 0 as $\epsilon \downarrow 0$ in two different frequency scales, $s$, and $\frac{s}{\epsilon}$. 


\section{Chapter 3}

\section{High Gain Stabilization for Single-Input Single-Output Systems}

The ability to design controllers of low dimensionality is critically related to the main result of this chapter. Since stability is always of initial priority, we provide here the means to stabilize a class of single-input single-output (SISO) systems using high gain control. This problem is more complicated than simply arbitrarily increasing the gain parameter. The order of our controller is intimately tied with the orders of infinite zeros of the system. We first show this connection here for the SISO case, where there is only a single order of infinite zero i.e. its relative degree. These necessary tools are extended to the multi-input multi-output (MIMO) case in the next chapter.

We wish to devise a controller for which stabilization is achieved for a family of SISO systems. Namely, the SISO family

$$
G_{r^{*}}=\left\{\alpha \frac{n(s)}{d(s)}: \alpha>0, n(s) \text { Hurwitz, } 0 \leq r \leq r^{*} \in \mathbb{N}\right\}
$$

where $n(s), d(s)$ are monic polynomials, and $r=\operatorname{deg} d(s)-\operatorname{deg} n(s) \geq 0 . G_{r^{*}}$ is then the set of all minimum phase systems of positive instantaneous gain with bounded relative degree.

Consider a controller of the following structure

$$
c(s, \epsilon)=\frac{1}{\epsilon^{p_{0}}} \frac{h(s)}{\prod_{i=1}^{l}\left(\epsilon^{p_{i}} s+1\right)}
$$

where $h(s)$ is a Hurwitz polynomial of degree $l$, and $p_{i} \in \mathbb{N}, i=0, \ldots, l$. Martensson 22 gave a specific choice for the $\left\{p_{i}\right\}$ in 3.2 which stabilizes the family $G_{r^{*}}$ for sufficiently small $\epsilon$. We seek a generalization of this for numerous reasons. The proof given in [22, although correct, does not provide insight into the asymptotic behaviour of the unbounded poles which characterize high gain systems. Further, arbitrary choices of $\left\{p_{i}\right\}$ can give rise to undesired behaviour such as instability as seen in [34, 22] which does not seem to have been fully examined in the literature. Lastly, this result plays a key role in the design of low order controllers.

We first state the main result of this chapter and then develop the necessary machinery for its proof in the sections to follow. 
Theorem 3.1. Consider $G_{r^{*}}$. For $l \geq r^{*}-1$, and $p_{0} \in \mathbb{N}$ there always exists a set of integers $\left\{p_{1}, p_{2}, \ldots, p_{l}\right\}$ such that the controller (3.2) stabilizes all systems $G(s) \in G_{r^{*}}$. In particular, the controller achieves this behaviour if

$$
p_{i} \geq \sum_{j=0}^{i-1} p_{j}
$$

for all $i=1, \ldots, l$.

Theorem 3.1 is a result on the simultaneous stabilization of $G_{r^{*}}$. Essentially, it is possible to stabilize the family of systems $G_{r^{*}}$ with controller 3.2 by choosing the order $l$, and the integers $p_{0}, \ldots, p_{l}$ appropriately.

Define the closed-loop characteristic polynomial of a system $G(s) \in G_{r^{*}}$ with controller 3.2 as $\Delta(\epsilon, s)$ where

$$
\Delta(\epsilon, s) \triangleq\left(\prod_{i=1}^{l}\left(\epsilon^{p_{i}} s+1\right)\right) d(s)+\frac{\alpha}{\epsilon^{p_{0}}} h(s) n(s) .
$$

We first determine the behaviour of the closed-loop poles of the system which remain finite before investigating the asymptotic properties of the remaining poles as $\epsilon \downarrow 0$.

\subsection{Behaviour of the Finite Poles of the Closed-loop System}

A standard root locus argument shows that the closed-loop poles which remain finite as the gain parameter $\frac{1}{\epsilon}$ tends to infinity terminate at the transmission zeros of the open loop system. This also holds true in the case of (3.4) as shown by the following.

Lemma 3.1. As $\epsilon \downarrow 0$, the closed-loop poles of 3.4) which remain finite tend to the roots of $h(s) n(s)$.

Proof. Since the second term of (3.4) dominates the first for $\epsilon$ sufficiently small on every bounded subset of $\mathbb{C}$, the proof follows trivially from continuity and Rouché's Theorem.

\subsection{Asymptotic Behaviour of the Closed-loop System}

The study of the asymptotic behaviour of the closed-loop system is far more complicated due to the structure of our controller 3.2 . We make use of the theory of algebraic functions (for details see for example [8]).

Definition 3.1. Let $\Delta(q, v)$ be a polynomial in $q$ of the form

$$
\Delta(q, v)=f_{0}(v) q^{m}+f_{1}(v) q^{m-1}+\cdots+f_{m}(v)
$$

where each coefficient $\left\{f_{i}(v): i=0, \ldots, m\right\}$ is itself a polynomial in $v$ with coefficients in $\mathbb{C}$. Then an algebraic function is a function $q(v)$ defined for values of $v \in \mathbb{C}$ by an equation of the form

$$
\Delta(q, v)=0
$$

Consider the closed-loop characteristic polynomial $\Delta(\epsilon, s)$ as in 3.4 , and let $g=\epsilon$. If we allow $g$ to be a complex number then the branches of $\Delta(g, s), s(g)$, is an algebraic function satisifying $\Delta(g, s)=0$. 
Since some of the roots of $\Delta(g, s)$ tend to infinity as $g \downarrow 0$, determining the asymptotic behaviour of the closed-loop system is equivalent to determining the asymptotic behaviour of each branch of $\Delta(g, s)$ around the point $s=\infty$.

The classical method of determining the behaviour of an algebraic function around a point is the Newton-Puiseux (NP) diagram approach, which was proposed by Newton in 1680, and re-examined by Puiseux in 1850 [24, 26]. Suppose that $\Delta(g, s)$ is a degree $n$ polynomial in $s$. It is well known that around $s_{0} \in \mathbb{C}$, the $n$ values of $s(g)$ are determined by a finite set of convergent (Puiseux) series, i.e. $\left\{s=\sum_{j=\delta_{i}}^{\infty} c_{i j} g^{\mu_{j}}: i=1, \ldots, m \leq n\right\}$. The NP diagram is a graphical tool which is used to determine the coefficients, and powers of the Puiseux series.

Consequently, the role in which the NP diagram serves here is to produce the first term in the series of each branch so that we may infer the angle at which it approaches the point of interest since, around the point of interest, subsequent terms in the series become negligible. This is precisely the technique used to compute the asymptotic behaviour of the multivariable root locus 25] from an algebraic function point of view. We will approach the use of this technique algorithmically.

\subsubsection{A Motivating Example}

Perhaps the simplest way to illustrate the use of NP diagrams is by example. For complete details on NP diagrams we refer the reader to [8]. Consider the standard root locus formulation for a strictly proper SISO system

$$
G(s)=\frac{n(s)}{d(s)}
$$

where,

$$
\begin{aligned}
& n(s)=b_{0} s^{m}+b_{1} s^{m-1}+\cdots+b_{m}, \\
& d(s)=s^{n}+a_{1} s^{n-1}+\cdots+a_{n}
\end{aligned}
$$

so that the closed-loop poles are the roots of

$$
\Delta(\epsilon, s)=\epsilon d(s)+n(s)=0 .
$$

We wish to re-prove that the asymptotic root locus forms a Butterworth pattern as in Chapter 2 .

Let $g=\epsilon \in \mathbb{C}$. We seek the asymptotic behaviour of the roots of $\Delta(g, s)$ around $s=\infty$. Thus, define the mapping $z=\frac{1}{s}$ so that

$$
\begin{aligned}
& \Delta(g, z)=z^{-n} \Psi(g, z), \\
& \Psi(g, z)=\left(g\left(1+\cdots+a_{n} z^{n}\right)+b_{0} z^{n-m}+\cdots+b_{m} z^{n}\right) .
\end{aligned}
$$

We seek an approximation of the series representation of each branch of the form $z(g)=c g^{\mu}$, where $c \in \mathbb{C}, \mu \in \mathbb{Q}$ are to be determined. Thus $\arg c$ will be the angle at which $z$ approaches 0 (or $s$ approaches $\infty)$, and $\mu$ is the speed in which it does so. The NP diagram technique allows us to accomplish this as follows.

Construct a plot with coordinate axis $g$, and ordinate axis $z$. A point $(x, y)$ on the NP diagram corresponds to the integer value of the powers in $g$, and $z$ respectively of each non-zero term in $\Psi(g, z)$ 


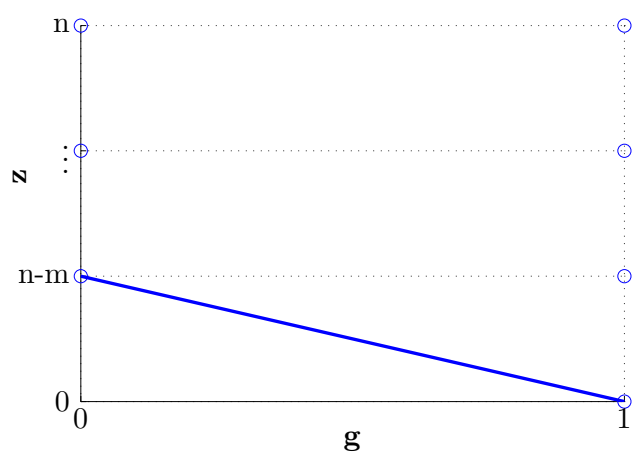

Figure 3.1: Newton-Puiseux diagram for the standard root locus formulation

so that we may construct Figure 3.1. For example, the point $(0, n-m)$ corresponds to the term $b_{0} z^{n-m}$, and $(1,0)$ to $g$ in 3.12 .

Definition 3.2. The lower convex hull of a Newton-Puiseux diagram corresponds to the boundary of the convex hull formed by connecting those points which lower bound the remaining points on the diagram. Each line segment of distinct slope on the boundary is denoted as a link. The sum of all terms from $\Psi(g, z)$ mapping to each point on a link is a link equation.

By standard arguments of the NP diagram construction [8, 25], the lower convex hull formed by connecting points $(0, n-m)$, and $(1,0)$, characterizes the branches of the algebraic function around the point of interest $(s=\infty)$. In particular, the number of branches of $\Delta(g, z)$ associated with a link is equal to the length of the orthogonal projection of that link onto the ordinate axis. For the case at hand, the single link in Figure 3.1 has $n-m$ associated branches. Furthermore, the most significant term of the Puiseux series of each branch associated with a link can be determined from its link equation. The points on the diagram above the lower convex hull are of no significance.

We are now ready to compute $c$, and $\mu$ from the lower convex hull. Consider, the link denoted by $l$ in Figure 3.1 or the line segment from $(0, n-m)$ to $(1,0)$. The link equation associated with $l$ must sum to zero [8], i.e.

$$
g+b_{0} z^{n-m}=0 .
$$

By the standard NP diagram construction, the quantity $\mu$ is defined to be the slope of the link $l$ with respect to the $z$ axis, i.e.

$$
\mu \triangleq-\frac{d}{d z} l=\frac{1}{n-m} .
$$

Substitution of $z=c g^{\mu}$ into the link equation 3.13 yields

$$
1+b_{0} c^{n-m}=0
$$

so that the arguments of the $n-m$ solutions in $c$ clearly give the angles of the asymptotes in the standard root locus.

Remark 3.1. The standard root locus results in a lower convex hull consisting of only a single link. This is not true for a general algebraic function which results in a specific approximation of the series representation of each branch, i.e. $z_{i}=c_{i} g^{\mu_{i}}$, for each link $l_{i}$. 


\subsubsection{Preliminary Development}

Consider now the full problem, where the closed-loop characteristic polynomial is given by (3.4). Using the intuition developed in the previous section, we shall show that the peculiar form of controller 3.2 is chosen to manipulate the lower convex hull associated with the NP diagram of the closed-loop system (3.4). In this way, we may directly manipulate the asymptotes along which the unbounded poles approach $s=\infty$. With stabilization in mind, it is desired to force the asymptotes to point into $\mathbb{C}_{-}$. The following result concerning the link equations of a NP diagram (see previous section) will be useful.

Proposition 3.1. Substituting $z=c g^{\mu}$ into any link equation $l$ where

$$
\mu \triangleq-\frac{d}{d z} l>0
$$

will always result in an equation that is a function of c only.

Proof. To see this, consider an arbitrary link equation of two terms (also true for any number of terms),

$$
0=g^{x} z^{y}+g^{p} z^{q}
$$

Since for each term above, the integral powers of $g$, and $z$ give the coordinates on the NP diagram to which they map, the slope of link between the two terms, i.e. $\mu$, is given by

$$
\mu=-\frac{x-p}{y-q}>0
$$

Substitution of $z=c g^{\mu}$ gives

$$
\begin{aligned}
0 & =g^{x+y \mu} c^{y}+g^{p+q \mu} c^{q} \\
& =g^{x+y \mu-q \mu+q \mu} c^{y}+g^{p+q \mu} c^{q} \\
& =g^{x+(y-q) \mu+q \mu} c^{y}+g^{p+q \mu} c^{q} \\
& =g^{p+q \mu} c^{y}+g^{p+q \mu} c^{q} \\
& =c^{y}+c^{q} .
\end{aligned}
$$

We now define several functions that will be helpful in expanding the closed-loop polynomial (3.4) for further analysis.

Definition 3.3. For a field $\boldsymbol{F}$, and a set $\left\{p_{1}, \ldots, p_{n}\right\}$ where $(\forall i) p_{i} \in \boldsymbol{F}$ let

$$
\left[\left\{p_{1}, \ldots, p_{n}\right\}\right]_{\Sigma} \triangleq p_{1}+p_{2}+\cdots+p_{n}
$$

Definition 3.4. For a field $\boldsymbol{F}$, and a set $\left\{p_{1}, \ldots, p_{n}\right\}$ where $(\forall i) p_{i} \in \boldsymbol{F}$ let

$$
\left\{p_{1}, \ldots, p_{n}\right\}_{i} \triangleq\left\{p_{j_{1}} p_{j_{2}} \ldots p_{j_{i}}: 1 \leq j_{1}<\cdots<j_{i} \leq n\right\}
$$

where

$$
\left\{p_{1}, \ldots, p_{n}\right\}_{0} \triangleq\{1\}
$$


Remark 3.2. $\left\{p_{1}, \ldots, p_{n}\right\}_{i}$ is then the set of all multiples of all combinations of $\left\{p_{1}, \ldots, p_{n}\right\}$ of size $i$. The next three propositions show that the above functions are well behaved.

Proposition 3.2. For a field $\boldsymbol{F}$, let $A, B \subset \boldsymbol{F}$ be sets with $A$, and $B$ disjoint. Then

$$
[A]_{\Sigma}+[B]_{\Sigma}=[A \cup B]_{\Sigma}
$$

Proof. Since $A$ and $B$ are disjoint

$$
\begin{aligned}
{[A]_{\Sigma}+[B]_{\Sigma} } & =\sum_{a_{i} \in A} a_{i}+\sum_{b_{i} \in B} b_{i} \\
& =\sum_{c_{i} \in A \cup B} c_{i} \\
& =[A \cup B]_{\Sigma} .
\end{aligned}
$$

Proposition 3.3. For a field $\boldsymbol{F}$, let $A=\left\{a_{1}, \ldots, a_{n}\right\}, B=\left\{b_{1}, \ldots, b_{m}\right\} \subset \boldsymbol{F}$ be sets. Then

$$
[A]_{\Sigma} \cdot[B]_{\Sigma}=[A \times B]_{\Sigma}
$$

Proof.

$$
\begin{aligned}
{[A]_{\Sigma} \cdot[B]_{\Sigma} } & =\left(a_{1}+\cdots+a_{n}\right) \cdot\left(b_{1}+\cdots+b_{m}\right) \\
& =\sum_{i=1}^{n} \sum_{j=1}^{m} a_{i} b_{j} \\
& =[A \times B]_{\Sigma} .
\end{aligned}
$$

Proposition 3.4. For a field $\boldsymbol{F}$, let $a \in \boldsymbol{F}$. Then $\left[\left\{a p_{1}, a p_{2}, \ldots, a p_{n}\right\}_{i}\right]_{\Sigma}=a^{i}\left[\left\{p_{1}, p_{2}, \ldots, p_{n}\right\}_{i}\right]_{\Sigma}$ Proof. Let $P=\left\{p_{1}, \ldots, p_{n}\right\}_{i}$ be the set of all multiples of all combinations of $\left\{p_{1}, \ldots, p_{n}\right\}$ of size $i$.

$$
\begin{aligned}
{\left[\left\{a p_{1}, a p_{2}, \ldots, a p_{n}\right\}_{i}\right]_{\Sigma} } & =\sum_{q_{j} \in P} a^{i} q_{j} \\
& =a^{i} \sum_{q_{j} \in P} q_{j} \\
& =a^{i}\left[\left\{p_{1}, p_{2}, \ldots, p_{n}\right\}_{i}\right]_{\Sigma}
\end{aligned}
$$

In order to expand the closed-loop characteristic polynomial (3.4), we require the following. 
Proposition 3.5. For distinct $p_{i}$,

$$
\prod_{i=1}^{n}\left(1+p_{i}\right)=\sum_{i=0}^{n}\left[\left\{p_{1}, \ldots, p_{n}\right\}_{i}\right]_{\Sigma} .
$$

Proof. We prove the claim by induction. For $n=1$ we have trivially

$$
\begin{aligned}
\left(1+p_{1}\right) & =\sum_{i=0}^{1}\left[\left\{p_{1}\right\}_{i}\right]_{\Sigma} \\
& =\left[\left\{p_{1}\right\}_{0}\right]_{\Sigma}+\left[\left\{p_{1}\right\}_{1}\right]_{\Sigma} \\
& =1+p_{1} .
\end{aligned}
$$

Suppose the claim holds for $n=j$. We then have

$$
\begin{aligned}
\prod_{i=1}^{j+1}\left(1+p_{i}\right) & =\left(1+p_{j+1}\right) \cdot \prod_{i=1}^{j}\left(1+p_{i}\right) \\
& =\left(1+p_{j+1}\right) \cdot \sum_{i=0}^{j}\left[\left\{p_{1}, \ldots, p_{j}\right\}_{i}\right]_{\Sigma} .
\end{aligned}
$$

Writing the above explicitly results in

$$
\begin{aligned}
\prod_{i=1}^{j+1}\left(1+p_{i}\right)= & \left(\left[\left\{p_{1}, \ldots, p_{j+1}\right\}_{0}\right]_{\Sigma}+\left[\left\{p_{j+1}\right\}_{1}\right]_{\Sigma}\right) \cdot\left[\left\{p_{1}, \ldots, p_{j}\right\}_{0}\right]_{\Sigma} \\
& +\left(\left[\left\{p_{1}, \ldots, p_{j+1}\right\}_{0}\right]_{\Sigma}+\left[\left\{p_{j+1}\right\}_{1}\right]_{\Sigma}\right) \cdot\left[\left\{p_{1}, \ldots, p_{j}\right\}_{1}\right]_{\Sigma} \\
& +\ldots \\
& +\left(\left[\left\{p_{1}, \ldots, p_{j+1}\right\}_{0}\right]_{\Sigma}+\left[\left\{p_{j+1}\right\}_{1}\right]_{\Sigma}\right) \cdot\left[\left\{p_{1}, \ldots, p_{j}\right\}_{j}\right]_{\Sigma}
\end{aligned}
$$

where by rearranging

$$
\begin{aligned}
= & {\left[\left\{p_{1}, \ldots, p_{j+1}\right\}_{0}\right]_{\Sigma} \cdot\left[\left\{p_{1}, \ldots, p_{j}\right\}_{0}\right]_{\Sigma} } \\
& +\left(\left[\left\{p_{j+1}\right\}_{1}\right]_{\Sigma} \cdot\left[\left\{p_{1}, \ldots, p_{j}\right\}_{0}\right]_{\Sigma}+\left[\left\{p_{1}, \ldots, p_{j+1}\right\}_{0}\right]_{\Sigma} \cdot\left[\left\{p_{1}, \ldots, p_{j}\right\}_{1}\right]_{\Sigma}\right) \\
& +\left(\left[\left\{p_{j+1}\right\}_{1}\right]_{\Sigma} \cdot\left[\left\{p_{1}, \ldots, p_{j}\right\}_{1}\right]_{\Sigma}+\left[\left\{p_{1}, \ldots, p_{j+1}\right\}_{0}\right]_{\Sigma} \cdot\left[\left\{p_{1}, \ldots, p_{j}\right\}_{2}\right]_{\Sigma}\right) \\
& +\ldots \\
& +\left(\left[\left\{p_{j+1}\right\}_{1}\right]_{\Sigma} \cdot\left[\left\{p_{1}, \ldots, p_{j}\right\}_{j-1}\right]_{\Sigma}+\left[\left\{p_{1}, \ldots, p_{j+1}\right\}_{0}\right]_{\Sigma} \cdot\left[\left\{p_{1}, \ldots, p_{j}\right\}_{j}\right]_{\Sigma}\right) \\
& +\left[\left\{p_{j+1}\right\}_{1}\right]_{\Sigma} \cdot\left[\left\{p_{1}, \ldots, p_{j}\right\}_{j}\right]_{\Sigma} .
\end{aligned}
$$

We claim that

$$
\left[\left\{p_{j+1}\right\}_{1}\right]_{\Sigma} \cdot\left[\left\{p_{1}, \ldots, p_{j}\right\}_{i}\right]_{\Sigma}+\left[\left\{p_{1}, \ldots, p_{j+1}\right\}_{0}\right]_{\Sigma} \cdot\left[\left\{p_{1}, \ldots, p_{j}\right\}_{i+1}\right]_{\Sigma}=\left[\left\{p_{1}, \ldots, p_{j+1}\right\}_{i+1}\right]_{\Sigma}
$$


Perhaps the easiest way to see this is to convert the claim into an equivalent form so that we may work with sets. We wish to apply Propositions 3.2 , and 3.3 . To use Proposition 3.2 it is required to show that

$$
\left(\left\{p_{j+1}\right\}_{1} \times\left\{p_{1}, \ldots, p_{j}\right\}_{i}\right) \bigcap\left(\left\{p_{1}, \ldots, p_{j+1}\right\}_{0} \times\left\{p_{1}, \ldots, p_{j}\right\}_{i+1}\right)=\phi
$$

where we have used Proposition 3.3. This follows immediately since the term $p_{j+1}$ does not appear in $\left\{p_{1}, \ldots, p_{j+1}\right\}_{0} \times\left\{p_{1}, \ldots, p_{j}\right\}_{i+1}=\left\{p_{1}, \ldots, p_{j}\right\}_{i+1}$.

Thus it is equivalent to show

$$
\left(\left\{p_{j+1}\right\}_{1} \times\left\{p_{1}, \ldots, p_{j}\right\}_{i}\right) \bigcup\left(\left\{p_{1}, \ldots, p_{j+1}\right\}_{0} \times\left\{p_{1}, \ldots, p_{j}\right\}_{i+1}\right)=\left\{p_{1}, \ldots, p_{j+1}\right\}_{i+1}
$$

Suppose that $x \in\left\{p_{j+1}\right\}_{1} \times\left\{p_{1}, \ldots, p_{j}\right\}_{i}$. This implies that

$$
x=p_{j+1} p_{x_{1}} \cdots p_{x_{i}}
$$

where the ordering is of no importance and $p_{x_{k}} \in\left\{p_{1}, \ldots, p_{j}\right\}$ are distinct for $k=1, \ldots, i$. Then certainly, $x \in\left\{p_{1}, \ldots, p_{j+1}\right\}_{i+1}$. We also have that

$$
x \in\left\{p_{1}, \ldots, p_{j+1}\right\}_{0} \times\left\{p_{1}, \ldots, p_{j}\right\}_{i+1}=\left\{p_{1}, \ldots, p_{j}\right\}_{i+1} \subset\left\{p_{1}, \ldots, p_{j+1}\right\}_{i+1} .
$$

Conversely, let

$$
x \in\left\{p_{1}, \ldots, p_{j+1}\right\}_{i+1} \Longrightarrow x=p_{x_{1}} p_{x_{2}} \cdots p_{x_{i+1}}
$$

for distinct $p_{x_{k}} \in\left\{p_{1}, \ldots, p_{j+1}\right\}, k=1, \ldots, i+1$. Suppose that, by arbitrary choice, the first multiple $p_{x_{1}}=p_{j+1}$. Then $x \in\left\{p_{j+1}\right\}_{1} \times\left\{p_{1}, \ldots, p_{j}\right\}_{i}$ by distinctness of the $p$ 's. If on the otherhand, for all $k, p_{x_{k}} \neq p_{j+1}$ then again by distinctness $x \in\left\{p_{1}, \ldots, p_{j}\right\}_{i+1}$. The claim and the proof are now complete.

It is possible now to determine those terms in 3.4 which will map to points on the lower convex hull of the NP diagram for $G(s) \in G_{r^{*}}$.

Lemma 3.2. Let $G(s)=\alpha \frac{n(s)}{d(s)}$ be a SISO system with relative degree $r>0, \Delta(g, z)$ be as in (3.4) where $g=\epsilon$ is allowed to be complex, and $z=\frac{1}{s}$. Suppose $l=r-1$, and $p_{0} \leq p_{1}<p_{2}<\cdots<p_{l}$. Then the terms which map to points on the lower convex hull of the Newton-Puiseux diagram of $\Delta(g, z)$ are given by

$$
\alpha z^{r}, g^{p_{0}} z^{l}, g^{p_{0}+p_{1}} z^{l-1}, \ldots, g^{\sum_{j=0}^{l-1} p_{j}} z, g^{\sum_{j=0}^{l} p_{j}}
$$

Proof. Manipulating $\Delta(g, z)$ using Proposition 3.4 , and 3.5 yields

$$
\Delta(g, z)=\frac{1}{g^{p_{0}}} \frac{1}{z^{l+n}} \Psi(g, z)
$$


where

$$
\Psi(g, z)=g^{p_{0}}\left(\sum_{i=0}^{l}\left[\left\{g^{p_{1}}, \ldots, g^{p_{l}}\right\}_{i}\right]_{\Sigma} z^{l-i}\right)\left(1+\cdots+a_{n} z^{n}\right)+\left(\alpha z^{r}+b_{1} z^{r+1}+\cdots+b_{l+n-r} z^{l+n}\right) .
$$

Consider $\Psi(g, z)$. Recall that the powers in $g, z$ of the non-zero terms in $\Psi(g, z)$ map to points on the NP diagram. Notice that the terms in $g$ are fixed in power and only vary in the power of $z$. This implies that the lower convex hull of the Newton-Puiseux diagram will remain unchanged if we replace $\left(1+\cdots+a_{n} z^{n}\right)$ with 1 since the additional powers in $z$ translate to points above the lower convex hull of which we are uninterested. For the same reason, we can replace $\left(\alpha z^{r}+b_{1} z^{r+1}+\cdots+b_{l+n-r} z^{l+n}\right)$ with $\alpha z^{r}$.

Of the $\left(\begin{array}{l}l \\ i\end{array}\right)$ terms in $g^{p_{0}}\left[\left\{g^{p_{1}}, \ldots, g^{p_{l}}\right\}_{i}\right]_{\Sigma} z^{l-i}$, by our choice of $\left\{p_{1}, \ldots, p_{l}\right\}$ the term that has the smallest power in $g$ is $g^{\sum_{j=0}^{i} p_{j}} z^{l-i}$ for $i=1, \ldots, l$. Additionally, let us index the list 3.37) as

$$
\left\{\alpha z^{r}, g^{p_{0}} z^{l}, g^{p_{0}+p_{1}} z^{l-1}, \ldots, g^{\sum_{j=0}^{l-1} p_{j}} z, g^{\sum_{j=0}^{l} p_{j}}\right\} \triangleq\left\{w_{0}, w_{1}, \ldots, w_{r}\right\} \triangleq W .
$$

Notice that for each $w_{i} \in W, w_{i}$ is the term with the lowest power of $g$ for a distinct power of $z$. The link equations associated with consecutive elements of $W$ are then

$$
l_{i}: \quad w_{i}+w_{i+1}=0
$$

for $i=0, \ldots, r-1$. If we explicitly calculate the slopes between each point on the NP diagram from which these terms map, we obtain

$$
\begin{aligned}
\mu_{0} \triangleq-\frac{d}{d z} l_{0} & =-\frac{p_{0}-0}{l-r}=p_{0} \\
\mu_{i} \triangleq-\frac{d}{d z} l_{i} & =-\frac{\sum_{j=0}^{i} p_{j}-\sum_{j=0}^{i-1} p_{j}}{l-i-(l-(i-1))}=p_{i}
\end{aligned}
$$

for $i=1, \ldots, r-1$. Since the slopes are monotonically increasing by our choice of $\left\{p_{i}\right\}$, the links $l_{i}$ for $i=0, \ldots, r-1$ must comprise the lower convex hull so that the elements of $W$ map to the points on the lower convex hull. To see this, suppose not, i.e. that there is a point, say $(p, t)$, on the lower convex hull which does not map to an element of $W$. Suppose further that this element $w_{x}=g^{p} z^{t} \notin W$, and let $w_{i}=g^{q} z^{t} \in W$ be the corresponding term in $W$ with power of $z$ equal to $t$. If $w_{x}$ is on the lower convex hull then necessarily $p \leq q$ since otherwise $w_{x}$ would belong to the interior of the convex hull (recall that the lower convex hull is the lower boundary of the convex hull formed by points on the NP diagram). But $w_{i}$ is the term with the smallest power in $g$ for which the power of $z$ is $t$. This implies that $p=q$, so that $w_{x}=w_{i}$ which is a contradiction.

Now that we have determined which terms map to points on the lower convex hull by the choice of $\left\{p_{i}\right\}$ it is then straightforward to determine each link equation from which the angles of the asymptotes can be computed. Although here there are multiple links, this is completely analogous to the standard root locus example developed previously. Thus the closed-loop asymptotic behaviour for any SISO plant $G(s)$ as defined in Lemma 3.2 after applying controller 3.2 is characterized by the following lemma.

Lemma 3.3. Let $\Delta(g, z),\left\{p_{0}, p_{1}, \ldots, p_{l}\right\}, l=r-1$, and $r$ be as in Lemma 3.2. Then the $r$ unbounded 
closed-loop poles tend to infinity along asymptotes which make angles arg $c_{i}$ with the positive real axis where $c_{i}$ is given by the solution of one of

$$
\begin{aligned}
\alpha c_{i}^{2}+c_{i}+1 & =0, \\
\alpha c_{i}+1 & =0, \\
c_{i}+1 & =0
\end{aligned}
$$

for $i=0, \ldots, r-1$.

Proof. Suppose $p_{0}<p_{1}$. Of the $r+1$ terms on the lower convex hull of the NP diagram of $\Delta(g, z)$, the link equations are the sum of any two consecutive terms in the ordering given by Lemma 3.2. These terms are given by

$$
\left\{\alpha z^{r}, g^{p_{0}} z^{l}, g^{p_{0}+p_{1}} z^{l-1}, \ldots, g^{\sum_{j=0}^{l-1} p_{j}} z, g^{\sum_{j=0}^{l} p_{j}}\right\} \triangleq\left\{w_{0}, w_{1}, \ldots, w_{r}\right\} \triangleq W
$$

so that the link equations are given by

$$
l_{i}: \quad w_{i}+w_{i+1}
$$

for $i=0, \ldots, r-1$. We also know from the proof of Lemma 3.2 that the slope of $l_{i}$ is equal to $\mu_{i}=p_{i}$. Thus on substitution of $z_{i}=c_{i} g^{\mu_{i}}$ into any link equation yields

$$
\begin{array}{ll}
l_{0}: & \alpha\left(c_{0} g^{\mu_{0}}\right)^{r}+g^{p_{0}}\left(c_{0} g^{\mu_{0}}\right)^{r-1}=\alpha c_{0}+1=0, \\
l_{i}: & g^{\sum_{j=0}^{i-1} p_{j}}\left(c_{i} g^{\mu_{i}}\right)^{r-i}+g^{\sum_{j=0}^{i} p_{j}}\left(c_{i} g^{\mu_{i}}\right)^{r-i-1}=c_{i}+1=0 .
\end{array}
$$

Since the slopes of each link $l_{i}$ is given by $\mu_{i}=p_{i}$, the case when $p_{0}=p_{1}$ gives exactly the above except that the first link $l_{0}$ is now comprised of three points since the slopes of the first two links are equal. Thus the resulting link equation for $l_{0}$ is given by $\alpha c_{i}^{2}+c_{i}+1=0$ from similar reasoning as above.

Recall that from the discussion on NP diagrams from the previous section the arguments of these solutions of $c_{i}$ associated with $l_{i}$ give the angles of the asymptotes to which the closed-loop poles asymptotically travel along. Thus the proof is complete.

We are now ready to prove Theorem 3.1 .

\subsection{Proof of Theorem 3.1}

Before we give the proof of the main theorem of this chapter, let us briefly discuss the approach. We have shown in the previous section that by Lemma 3.3. if the integers $\left\{p_{i}^{(r)}\right\}$, and $l^{(r)}$ are chosen appropriately, we can ensure that for $G(s) \in G_{r^{*}}$ of a particular relative degree $r \leq r^{*}$, the unbounded closed-loop poles tend to infinity within $\mathbb{C}_{-}$. The superscript is added to emphasize the dependence on $r$. The integers $\left\{p_{i}^{(r)}\right\}$ are specifically chosen to manipulate the lower convex hull of the NP diagram associated with $G(s)$ to ensure that the asymptotes (along which the unbounded poles travel) point into $\mathbb{C}_{-}$. We also know from Lemma 3.1 that those poles which remain finite tend to the transmission zeros of the open loop system which are assumed to be stable. These tools are sufficient to stabilize any $G(s) \in G_{r^{*}}$ for any fixed $r$. 
In order to prove Theorem 3.1, we are constrained to choose a single $l$, and one set $\left\{p_{i}\right\}$ so that the entire family $G_{r^{*}}$ can be stabilized for all relative degrees less than or equal to $r^{*}$. We aim to show that it is possible to choose these parameters of the controller so that the unbounded poles of the closed-loop system can be characterized by Lemma 3.3 for any $G(s) \in G_{r^{*}}$.

Proof. Let $l=r^{*}-1$. This can be done without loss of generality since if $l>r^{*}-1$ then $r^{*}$ can be appropriately redefined to be a larger integer $p^{*}$ so that stabilizing $G_{p^{*}}$ will stabilize $G_{r^{*}}$ since $G_{r^{*}} \subset G_{p^{*}}$.

The closed-loop poles which remain finite tend to the roots of $h(s) n(s)$ by Lemma 3.1 which are stable by the minimum phase assumption and the structure of the controller (3.2). We also know from Lemma 3.3 that for systems $G(s) \in G_{r^{*}}$ of relative degree $\mathrm{r}$, if $p_{0}^{(r)} \leq p_{1}^{(r)}<p_{2}^{(r)}<\cdots<p_{r-1}^{(r)}$ then the controller 3.2 forces the unbounded closed-loop poles to travel to infinity along asymptotes that point into $\mathbb{C}_{-}$. Thus, we already have the tools to stabilize the plant for a particular relative degree $r$.

However, we are constrained to choose only one set of $P \triangleq\left\{p_{0}, p_{1}, \ldots, p_{l}\right\}$ which stabilizes all plants in $G_{r^{*}}$ where the relative degree is not fixed but ranges from 0 to $r^{*}$. We will construct the set of integers $P$ that achieves this and show that for any $r \leq r^{*}, P_{r} \triangleq\left\{p_{0}^{(r)}, \ldots, p_{r-1}^{(r)}\right\}$ is a linear combination of elements of $P$ that satisfy Lemma 3.3 . In this way, regardless of the relative degree, the closed-loop system is always stable for sufficiently small $\epsilon$.

Let us now construct the set $P$. Suppose $r=r^{*}$, and $p_{0} \in \mathbb{N}$ is given. Recall that $l=r^{*}-1$. An example of the following construction for $r^{*}=5$ is given in Figure 3.2. From Lemma 3.2, the terms which map to points on the lower convex hull of the NP diagram are

$$
\alpha z^{r}, g^{p_{0}} z^{l}, g^{p_{0}+p_{1}} z^{l-1}, \ldots, g^{\sum_{j=0}^{l-1} p_{j}} z, g^{\sum_{j=0}^{l} p_{j}}
$$

Recall also that these terms map to the lower convex hull of the NP diagram according to the powers in $z$, and $g$.

Label the point $\left(0, r^{*}\right)$ as $X_{0}$ (where $X_{0}$ corresponds to the term $\alpha z^{r}$ from (3.51)). Draw the constraint line, $C_{1}$, from $\left(0, r^{*}\right)$ through $X_{1}=\left(p_{0}, r^{*}-1\right)$ (where $X_{1}$ corresponds to the term $g^{p_{0}} z^{l}$ from 3.51$)$. Note $X_{1}$ is fixed for given $p_{0}$, and denote the link from $X_{0}$ to $X_{1}$ as $l_{0}$. We choose $p_{1}$ so that the next point on the lower convex hull, $X_{2}=\left(p_{0}+p_{1}, r^{*}-2\right)$ (where $X_{2}$ corresponds to the term $\left.g^{p_{0}+p_{1}} z^{l-1}\right)$, lies on or to the right of $C_{1}$. If $X_{2}$ is to the left of $C_{1}$ then $X_{1}$ would no longer be on the lower convex hull for $r=r^{*}$. Denote the line segment from $X_{1}$ to $X_{2}$ as $l_{1}$. Since the equation of the line associated with $C_{1}$ is

$$
g \geq-p_{0} z+p_{0} r^{*}
$$

substituting $g=p_{0}+p_{1}, z=r^{*}-2$ corresponds to choosing

$$
p_{1} \geq p_{0}
$$

Suppose now $r=r^{*}-1$. We construct the constraint line, $C_{2}$, by drawing the line from $\left(0, r^{*}-1\right)$ through $X_{2}$. We choose $p_{2}$ such that the next point on the lower convex hull, $X_{3}=\left(p_{0}+p_{1}+p_{2}, r^{*}-3\right)$ is on or to the right of $C_{2}$. If $X_{3}$ is to the left of $C_{2}$ then $X_{2}$ would no longer be on the lower convex hull for $r=r^{*}-1$. Denote the third link, $l_{2}$, as the line segment between $X_{2}$ and $X_{3}$. Since the equation of the line associated with $C_{2}$ is given by

$$
g \geq-\left(p_{0}+p_{1}\right) z+\left(p_{0}+p_{1}\right)\left(r^{*}-1\right)
$$




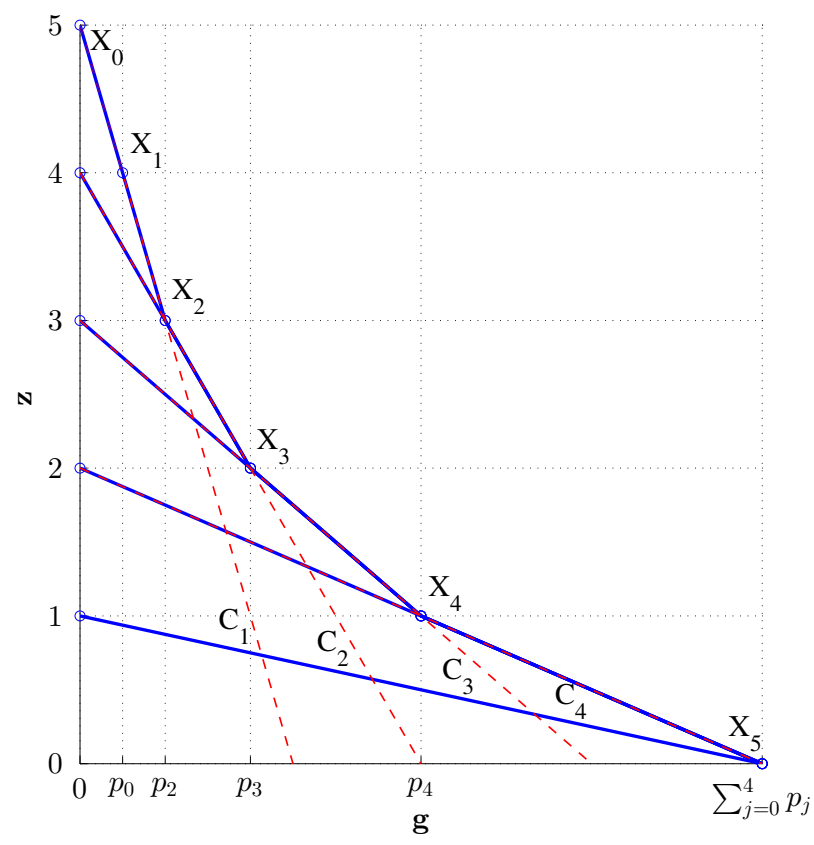

Figure 3.2: Newton-Puiseux diagram for $\Delta(g, z), r^{*}=5$

substitution of $g=p_{0}+p_{1}+p_{2}, z=r^{*}-3$ shows that the above requirement is then equivalent to

$$
p_{2} \geq p_{1}+p_{0}
$$

Then $r$ is repeatedly lowered to yield the following iterative equation for the constraint associated with $C_{i}$

$$
g \geq-\left(\sum_{j=0}^{i-1} p_{j}\right) z+\left(\sum_{j=0}^{i-1} p_{j}\right)\left(r^{*}-(i-1)\right),
$$

so that substitution of $g=p_{i}+\sum_{j=0}^{i-1} p_{j}, z=\left(r^{*}-(i+1)\right)$ results in equation 3.3 which places the point $X_{i+1}$ onto or to the right of $C_{i}$, for $i=1, \ldots, l$. The last point, $X_{r^{*}}$ is located at $\left(\sum_{i=0}^{l} p_{i}, 0\right)$.

We must now verify that by this choice of $P$, Lemma 3.3 is satisfied for any $r \leq r^{*}$. For such a $r$, it is clear from the above that the terms on the corresponding lower convex hull of the NP diagram would be those terms from 3.51 that have powers of $z$ strictly less than $r$. Denote $X_{0}^{(r)}$ as the point $(0, r)$ on the lower convex hull corresponding to the term $\alpha z^{r}$ from 3.51). Thus the next point $X_{1}^{(r)}$ with power one less than $r$ is $\left(\sum_{j=0}^{r^{*}-r} p_{j}, r-1\right)$ corresponding to the term $g^{\sum_{j=0}^{r^{*}-r} p_{j}} z^{r-1}$. The correspondence between $X_{i}^{(r)}$, and $X_{i}$ is then simply $X_{i}^{(r)}=X_{i+\left(r^{*}-r\right)}$ for $i>0$, and $X_{0}^{(r)}=(0, r)$ for $i=0$. With the original construction of the lower convex hull using $P$ in mind, this is equivalent to choosing

$$
\begin{gathered}
p_{0}^{(r)}=\sum_{j=0}^{r^{*}-r} p_{j}, \\
p_{i}^{(r)}=p_{i+\left(r^{*}-r\right)}
\end{gathered}
$$


for $i=1, \ldots, r-1$. By assumption

$$
p_{i} \geq \sum_{j=0}^{i-1} p_{j}
$$

for $i=1, \ldots, r^{*}-1$, and given $p_{0} \in \mathbb{N}$ which implies

$$
p_{0}^{(r)} \leq p_{1}^{(r)}<\cdots<p_{r-1}^{(r)}
$$

so that from Lemma 3.3 the closed-loop system is stable for any relative degree $r \leq r^{*}$.

Remark 3.3. An example of the NP diagram for systems in $G_{r^{*}}$ for $r^{*}=5$ is given in Figure 3.2 where equality has been taken in (3.3). The line beginning from $(0,3)$ and terminating on the $g$ axis is the lower convex hull of the closed-loop system for $G(s) \in G_{r^{*}}$ of relative degree 3 . The remaining lines follow a similar description. Note that the higher $r^{*}$ becomes, the size of the $\left\{p_{i}\right\}$ grows quite quickly as shown by the plot.

Remark 3.4. Indeed, if we choose $p_{0}=1$, and $p_{i}=2^{i}$, which can be easily verified to satisfy (3.3), we then recover Martensson's original controller.

\subsection{Further Developments}

Now consider the following controller

$$
c(s, \epsilon)=\frac{\theta}{\epsilon^{p_{0}}} \frac{h(s)}{\prod_{i=1}^{l}\left(\epsilon^{p_{i}} s+1\right)}
$$

where the $\theta \in \mathbb{R}_{++}$parameter is inserted to adjust the high frequency gain of the plant. The adjustment of $\theta$ allows one to force all the unbounded poles to approach $s=\infty$ along the negative real axis as shown in the following theorem. This implies that a well-damped closed-loop system can be attained by our controller in contrast to standard root locus design methods when the relative degree is strictly larger than 1.

Theorem 3.2. For $l \geq r^{*}-1$, and $p_{0} \in \mathbb{N}$ there always exists a set of integers $\left\{p_{1}, p_{2}, \ldots, p_{l}\right\}$, and $\theta \in \mathbb{R}_{++}$such that the controller 3.61) stabilizes all systems $G(s) \in G_{r^{*}}$, and the unbounded poles of the closed-loop system tend to infinity along the negative real axis. In particular, the controller achieves this behaviour if

$$
p_{i} \geq \sum_{j=0}^{i-1} p_{j}
$$

for all $i=1, \ldots, l$. For $G(s) \in G_{r^{*}}$ of relative degree $r$, as $\epsilon \downarrow 0$ the poles of the closed-loop poles tend to the transmission zeros of the system and to

$$
\begin{aligned}
& s_{0}=-\frac{1}{\epsilon^{\sum_{j=0}^{r^{*}-r} p_{j}}}, \\
& s_{i}=-\frac{1}{\epsilon^{p_{i+\left(r^{*}-r\right)}}}
\end{aligned}
$$

for $i=1, \ldots, r-1$. 
Proof. Begin with the construction of the Newton-Puiseux diagram as in Theorem 3.1. Consider the resulting link equations of the form

$$
\begin{aligned}
& 0=c_{i}+1, \\
& 0=\theta \alpha c_{1}^{2}+c_{1}+1
\end{aligned}
$$

for $i=3, \ldots, r$ if constraints 3.62 are met with equality, and

$$
\begin{aligned}
& 0=c_{i}+1, \\
& 0=\theta \alpha c_{1}+1
\end{aligned}
$$

for $i=2, \ldots, r$ if constraints 3.62 are met with strict inequality. Having the unbounded poles tend to infinity along the negative real axis is equivalent to having real and negative solutions of $c_{i}$. The first case where 3.62 are met with strict inequality follows immediately. For the other case, the first equation (3.65), $c_{i}$ is always real, and negative. For the second equation (3.66), if $\theta$ is adjusted such that

$$
1>4 \theta \alpha
$$

$c_{i}$ is guaranteed to be real, and is also negative by the Hurwitz criterion. The claim on the speeds of the unbounded poles follows directly by noting that for the approximation of each branch $z_{i}=c_{i} g^{\mu_{i}}$ associated with link $l_{i}$,

$$
\mu_{i}=-\frac{d}{d z} l_{i}=p_{i}
$$

as was done similarly in the proof of Theorem 3.1. The proof is complete since $s_{i}=\frac{1}{z_{i}}$, and $g=\epsilon$.

Remark 3.5. In some sense Theorem 3.2 allows one to control the angles of the asymptotes of the non-standard, multi-parameter root locus of the closed-loop system.

For the case of SISO systems, the relative degree is the (only) order of infinite zero. However, MIMO systems in general can possess multiple orders of infinite zeros. The following result will be useful in the next section. Define

$$
\Gamma=\left\{r_{1}, \ldots, r_{x}\right\} \subset\left\{1, \ldots, r^{*}\right\}
$$

and the following subset of $G_{r^{*}}$

$$
G_{\Gamma}=\left\{G(s) \in G_{r^{*}}: r \in \Gamma\right\}
$$

where $r$ is the relative degree of $G(s)$.

Theorem 3.3. Let $G_{\Gamma} \subset G_{r^{*}}$ be as above, and $l \geq r^{*}-1$. Then the controller (3.2), or (3.61) achieves Theorem 3.1, or 3.2 respectively for all $G(s) \in G_{\Gamma}$ if

$$
\begin{cases}p_{i} \geq \sum_{j=0}^{i-1} p_{j} & \text { if } i \in \Gamma \\ p_{i}>p_{i-1} & \text { else }\end{cases}
$$

for $i=1, \ldots, l$.

Proof. Begin with the construction of the NP diagram as in Theorem 3.1. The $r_{i} \notin \Gamma$ correspond to the absence of constraint line $C_{r_{i}}$. Thus, choosing $p_{r_{i}}>p_{r_{i-1}}$ as the weaker constraint ensures that Lemma 
3.3 is still satisfied. The remainder follows from the arguments of Theorems 3.1 and 3.2 .

\subsection{A Lower Bound on the Order of the Controller}

The choice of the order $l$ of the controller (3.2), and (3.61) can also be characterized using the exact same methodology as the previous sections. Thus we attain the following lower bounds on the dynamic order of the controller.

Corollary 3.1. The controller (3.2) achieves Theorem 3.1 only if the order of the controller $l \geq r^{*}-2$.

Proof. Suppose not. Let $l=r^{*}-3$. Begin with the construction of the Newton-Puiseux diagram as in Theorem 3.1. This corresponds to shifting the points on the Newton-Puiseux diagram which are not on the line $g=0$ down by two. Hence the equation associated with the first link (determined as the sum of the first two terms in Lemma 3.2 when $r=r^{*}$ has the form

$$
0=\alpha c_{1}^{3}+1
$$

This contradicts that the solutions of $c_{1}$ have negative real parts since the arguments of $c_{1}$ are the angles of the asymptotes that the unbounded closed-loop poles travel along which were assumed to point into $\mathbb{C}_{-}$.

Corollary 3.2. The controller (3.61) achieves Theorem 3.2 only if the order of the controller $l \geq r^{*}-1$.

Proof. Suppose not. Let $l=r^{*}-2$. Begin with the construction of the Newton-Puiseux diagram as in Theorem 3.1. This corresponds to shifting the points on the Newton-Puiseux diagram which are not on the line $g=0$ down by one. Hence the equation associated with the first link (determined as the sum of the first two terms in Lemma 3.2 when $r=r^{*}$ has the form

$$
0=\theta \alpha c_{1}^{2}+1 .
$$

This contradicts that $c_{1}$ is real valued since $\theta \alpha>0$. 


\section{Chapter 4}

\section{Perfect Regulation with Bounded Peaking}

We seek in this chapter to solve the perfect regulation problem with bounded peaking for multi-input multi-output (MIMO), invertible and minimum phase linear time-invariant systems. It is then necessary to first ensure that the closed-loop system can be stabilized by the proposed controller design. In the previous chapter, it was shown that for minimum phase systems of bounded relative degree, the stabilization problem is solvable using Theorem 3.1. and controller 3.2 . The MIMO case is inherently more complicated. The notion of relative degree, as will be seen, will be replaced by the orders of infinite zeros. This of course specializes back to the SISO case where the single order of infinite zero is equivalent to the relative degree.

Our methodology revolves around the multivariable root locus approach from Chapter 2 , and the results developed from the previous chapter. The aim then in this chapter is to obtain a high gain stabilization result in similar fashion for the MIMO system case. We first develop a general result on the product of Smith-McMillan forms to be used in subsequent sections. A high gain approximation for the poles of the closed-loop system is then obtained and the Simple Null Structure assumption from the multivariable root locus literature is discussed (see also Chapter 21. The high gain stabilization result and perfect regulation with bounded peaking follows (almost) immediately.

\subsection{On The Smith-McMillan Form}

The goal of this section is to provide conditions under which the Smith-McMillan form of the product of transfer matrices is equal to the product of the Smith-McMillan form of each transfer matrix, i.e. for a field $\mathbf{F}$

$$
\left(A, B \in \mathbf{F}^{n \times n}\right) \quad D_{M}(A B)=D_{M}(A) D_{M}(B)
$$

where we have borrowed the notation from Chapter 2 This result will be useful in the subsequent section.

The following result from Newman 23] concerning the Smith Normal Form is needed. Let $\mathbf{R}$ be a principal ideal domain. 
Theorem 4.1. 23] Suppose that $A, B \in \boldsymbol{R}^{n \times n}$ have relatively prime determinants. Then,

$$
D(A B)=D(A) D(B) .
$$

From this we can infer the main result of this section.

Theorem 4.2. Let $\boldsymbol{R}$ be a principal ideal domain, and $\boldsymbol{F}$ the field of fractions associated with $\boldsymbol{R}$. Suppose that for $A, B \in \boldsymbol{F}^{n \times n}, \Psi_{A}, \Phi_{A}, \Psi_{B}, \Phi_{B}$ are the denominator and numerator factors in the Smith-McMillan sense respectively. If $\Psi_{A} \Phi_{A}$, and $\Psi_{B} \Phi_{B}$ are relatively prime, then

$$
D_{M}(A B)=D_{M}(A) D_{M}(B)
$$

Proof. Let $A, B \in \mathbf{F}^{n \times n}$ so that

$$
\begin{aligned}
& A=P_{A} D_{M}(A) Q_{A} \\
& =P_{A}\left[\begin{array}{ccc}
\frac{\epsilon_{1 A}}{\psi_{1 A}} & & \\
& \ddots & \\
& & \frac{\epsilon_{n A}}{\psi_{n A}}
\end{array}\right] Q_{A} \\
& =\frac{1}{\Psi_{A}} P_{A}\left[\begin{array}{lll}
\phi_{1 A} & & \\
& \ddots & \\
& & \phi_{n A}
\end{array}\right] Q_{A} \\
& =\frac{1}{\Psi_{A}} M_{A}
\end{aligned}
$$

and similarly for $B$. Note that $M_{A}, M_{B} \in \mathbf{R}^{n \times n}$. Since the divisors of $\operatorname{det} M_{A}$, and $\operatorname{det} M_{B}$ belong respectively to the divisors of $\Psi_{A} \Phi_{A}$, and $\Psi_{B} \Phi_{B}$ which by assumption are relatively prime, we have by Theorem 4.1 that

$$
\begin{aligned}
D_{M}(A B) & =\frac{1}{\Psi_{A} \Psi_{B}} D\left(M_{A} M_{B}\right) \\
& =\frac{1}{\Psi_{A} \Psi_{B}} D\left(M_{A}\right) D\left(M_{B}\right) \\
& =\frac{1}{\Psi_{A} \Psi_{B}}\left[\begin{array}{lll}
\phi_{1 A} \phi_{1 B} & & \\
& \ddots & \\
& & \phi_{n A} \phi_{n B}
\end{array}\right] \\
& =\left[\begin{array}{ccc}
\frac{\epsilon_{1 A}}{\psi_{1 A}} \frac{\epsilon_{1 B}}{\psi_{1 B}} & & \\
& \ddots & \\
& & \frac{\epsilon_{n A}}{\psi_{n A}} \frac{\epsilon_{n B}}{\psi_{n B}}
\end{array}\right] \\
& =D_{M}(A) D_{M}(B) .
\end{aligned}
$$

and the proof is complete.

From a control point of view, the above theorem essentially states the following.

Corollary 4.1. For transfer matrices $A, B \in \mathbb{R}(s)^{m \times m}$, if the poles and zeros of $A$, and those of $B$ are 
disjoint, then

$$
D_{M}(A B)=D_{M}(A) D_{M}(B)
$$

\subsection{A High Gain Approximation}

Our aim in this section is to show that, with proper controller design, the closed-loop system under high gain control can be approximated by several decoupled SISO systems.

Towards this end, we re-state Theorem 6.3 of Hung \& MacFarlane [10] as the following Lemma. This lemma allows one to decompose a transfer matrix based on its infinite zero structure and allows for further analysis.

Lemma 4.1. ([10]) Suppose $G(s) \in \mathbb{R}(s)^{m \times m}$ is invertible. Then there exists non-singular matrices $U, Y \in \mathbb{R}^{m \times m}$ of the form

$$
\begin{aligned}
U & =\left[\begin{array}{llll}
U^{1} & U^{2} & \cdots & U^{v}
\end{array}\right] \\
Y & =\left[\begin{array}{llll}
Y^{1} & Y^{2} & \cdots & Y^{v}
\end{array}\right]
\end{aligned}
$$

where

$$
\begin{aligned}
U^{i} & := \begin{cases}R_{1}^{1} & \text { if } i=1 \\
R_{2}^{1} R_{2}^{2} \ldots R_{2}^{i-1} R_{1}^{i} & \text { if } i>1\end{cases} \\
Y^{i} & := \begin{cases}P_{1}^{1} & \text { if } i=1 \\
P_{2}^{1} P_{2}^{2} \ldots P_{2}^{i-1} P_{1}^{i} & \text { if } i>1\end{cases}
\end{aligned}
$$

so that

$$
\begin{aligned}
& Y^{T} G(s) U=\left[\begin{array}{cc}
\frac{1}{s^{\sigma_{1}}} \Sigma_{1}+\frac{1}{s^{\sigma_{1}+1}} W_{1}(s) & \frac{1}{s^{\sigma_{1}+1}} X_{1}(s) \\
\frac{1}{s^{\sigma_{1}+1}} Y_{1}(s) & \frac{1}{s^{\sigma_{1}+2}} Z_{1}(s)
\end{array}\right]+\cdots \\
& +\left[\begin{array}{cc}
0_{m-d_{i-1}} & 0 \\
0 & {\left[\begin{array}{cc}
\frac{1}{s^{\sigma_{i}}} \Sigma_{i}+\frac{1}{s^{\sigma_{i}+1}} W_{i}(s) & \frac{1}{s^{\sigma_{i}+1}} X_{i}(s) \\
\frac{1}{s^{\sigma_{i}+1}} Y_{i}(s) & \frac{1}{s^{\sigma_{i}+2}} Z_{i}(s)
\end{array}\right]}
\end{array}\right]+\cdots \\
& +\left[\begin{array}{cc}
0_{m-d_{v-1}} & 0 \\
0 & \frac{1}{s^{\sigma_{v}}} \Sigma_{v}+\frac{1}{s^{\sigma_{v}+1}} W_{v}(s)
\end{array}\right]
\end{aligned}
$$

and

$$
\frac{1}{s^{\sigma_{i}+2}} Z_{i}(s)-\frac{1}{s^{\sigma_{i}+1}} Y_{i}(s)\left(\frac{1}{s^{\sigma_{i}}} \Sigma_{i}+\frac{1}{s^{\sigma_{i}+1}} W_{i}(s)\right)^{-1} \frac{1}{s^{\sigma_{i}+1}} X_{i}(s)=0
$$

for $i=1, \ldots, v$. The matrices $\left\{\Sigma_{i}: i=1, \ldots, v\right\}$ are diagonal and contain strictly positive entries. Namely, they are the singular values of the PMPs of the system. $W_{i}(s), X_{i}(s), Y_{i}(s), Z_{i}(s)$ are all proper transfer matrices of appropriate dimensions. The integers $\left\{\sigma_{i}: 1 \leq \sigma_{1}<\cdots<\sigma_{v}, i=1, \ldots, v\right\}$ are the 
orders of infinite zeros of $G(s)$.

Lemma 4.2. [10] The series in Lemma[4.1 terminates at a finite step if and only if $G(s)$ is invertible.

Remark 4.1. Computation of the matrices $U, Y$ is straightforward and given in [10]. They are computed in much the same way as the multivariable root locus development in Chapter 2 except, in place of the eigenvalue decomposition, the singular value decomposition is used.

Lemmas 4.1, and 4.2 are critical in developing the MIMO generalization of the high gain stabilization result from Chapter 3. Lemma 4.1 is in essence a decomposition of a system based on its infinite zero structure, and Lemma 4.2 indicates precisely when this structure is well behaved. Each term in the decomposition denotes the presence of a particular order of infinite zero. Since this structure is heavily exploited, we assume invertibility throughout the remainder of this chapter as done previously.

We give a simple example on the computation of the constant matrices $U, Y$ of Lemma 4.1 .

Example 4.1. Consider the system

$$
G(s)=\left[\begin{array}{ccc}
0 & \frac{1}{s} & 0 \\
0 & 0 & \frac{1}{s^{2}} \\
\frac{1}{s^{2}} & 0 & 0
\end{array}\right]
$$

which has the following realization

$$
\begin{gathered}
\dot{x}(t)=\left[\begin{array}{lllll}
0 & 0 & 0 & 0 & 0 \\
1 & 0 & 0 & 0 & 0 \\
0 & 0 & 0 & 0 & 0 \\
0 & 0 & 0 & 0 & 0 \\
0 & 0 & 0 & 1 & 0
\end{array}\right] x(t)+\left[\begin{array}{lll}
1 & 0 & 0 \\
0 & 0 & 0 \\
0 & 1 & 0 \\
0 & 0 & 1 \\
0 & 0 & 0
\end{array}\right] u(t), \\
y(t)=\left[\begin{array}{lllll}
0 & 0 & 1 & 0 & 0 \\
0 & 0 & 0 & 0 & 1 \\
0 & 1 & 0 & 0 & 0
\end{array}\right] x(t) .
\end{gathered}
$$

From Chapter 2, Theorem 2.9 (where we have replaced the eigenvalue decomposition with the singular value decomposition), it is easy to verify that the singular value decomposition of the first PMP of the system is

$$
\begin{aligned}
G_{1}=C B \triangleq C_{1} B_{1} & =\left[\begin{array}{lll}
0 & 1 & 0 \\
0 & 0 & 0 \\
0 & 0 & 0
\end{array}\right] \\
& =\left[\begin{array}{lll}
1 & 0 & 0 \\
0 & 0 & 1 \\
0 & 1 & 0
\end{array}\right]\left[\begin{array}{lll}
1 & 0 & 0 \\
0 & 0 & 0 \\
0 & 0 & 0
\end{array}\right]\left[\begin{array}{ccc}
0 & 0 & -1 \\
1 & 0 & 0 \\
0 & 1 & 0
\end{array}\right] \\
& \triangleq\left[\begin{array}{ll}
P_{1}^{1} & P_{2}^{1}
\end{array}\right]\left[\begin{array}{cc}
\Sigma_{1} & 0 \\
0 & 0 \\
d_{1}
\end{array}\right]\left[\begin{array}{l}
R_{1}^{1 T} \\
R_{2}^{1 T}
\end{array}\right] .
\end{aligned}
$$


The singular value decomposition of the second PMP is then

$$
\begin{aligned}
G_{2} \triangleq C_{2} A_{2} B_{2} & =P_{2}^{1 T} C_{1}\left(I_{5}-B_{1} P_{1}^{1} \Sigma_{1}^{-1} R_{1}^{1 T} C_{1}\right) A_{1} B_{1} R_{2}^{1} \\
& =\left[\begin{array}{cc}
0 & -1 \\
1 & 0
\end{array}\right] \\
& =\left[\begin{array}{cc}
0 & -1 \\
-1 & 0
\end{array}\right]\left[\begin{array}{cc}
1 & 0 \\
0 & 1
\end{array}\right]\left[\begin{array}{cc}
-1 & 0 \\
0 & 1
\end{array}\right] \\
& \triangleq P_{1}^{2} \Sigma_{2} R_{1}^{2 T} .
\end{aligned}
$$

Thus, define

$$
\begin{aligned}
Y^{T} & =\left[\begin{array}{cc}
I_{d_{1}} & 0 \\
0 & P_{1}^{2 T}
\end{array}\right]\left[\begin{array}{c}
P_{1}^{1 T} \\
P_{2}^{1 T}
\end{array}\right], \\
U & =\left[\begin{array}{ll}
R_{1}^{1} & R_{2}^{1}
\end{array}\right]\left[\begin{array}{cc}
I_{d_{1}} & 0 \\
0 & R_{1}^{2}
\end{array}\right]
\end{aligned}
$$

so that

$$
Y^{T} G(s) U=\left[\begin{array}{ccc}
\frac{1}{s} & 0 & 0 \\
0 & \frac{1}{s^{2}} & 0 \\
0 & 0 & \frac{1}{s^{2}}
\end{array}\right]
$$

which is in the form as given by Lemma 4.1.

Consider the controller

$$
C(s, \epsilon)=c(s, \epsilon) U Y^{T}
$$

where the dynamical portion of the controller is given by (3.2), or 3.61) and the constant matrices $U, Y$ are given by Lemma 4.1. Recall that

$$
c(s, \epsilon)=\frac{1}{\epsilon^{p_{0}}} \frac{h(s)}{\prod_{i=1}^{l}\left(\epsilon^{p_{i}} s+1\right)}
$$

where $h(s)$ is a Hurwitz polynomial of degree $l$, and $p_{i} \in \mathbb{N}, i=0, \ldots, l$. The order of $c(s, \epsilon)$ is then $l$. We further assume, without loss of generality that $p_{0}=1$. The motivation for the above controller structure will become apparent in the following development.

Definition 4.1. [10] A system with a strictly proper transfer-function matrix $G(s) \in \mathbb{R}(s)^{m \times m}$ is said to have a cluster of $\sigma_{i}^{\text {th }}$-order unbounded asymptotic root locus (UARL) if there are $\sigma_{i}$ branches $\left\{s_{j}\left(\frac{1}{\epsilon}\right): j=1, \ldots, \sigma_{i}\right\}$ of root loci and $\lambda_{i}(\neq 0) \in \mathbb{C}$ such that,

$$
\lim _{\epsilon \downarrow 0} \epsilon s_{j}^{\sigma_{i}}=\lambda_{i} .
$$

Lemma 4.3. [10] If a strictly proper $G(s) \in \mathbb{R}(s)^{m \times m}$ is invertible and satisfies Simple Null Structure (see Definition 2.20) then the number of clusters of $\sigma_{i}^{\text {th }}$-order UARL is equal to the number of $\sigma_{i}^{\text {th }}$-order infinite zeros of $G(s)$. 
Remark 4.2. The UARL as given by Definition 4.1 can be interpreted as the root loci traced by the closed-loop poles as $\epsilon \downarrow 0$. Lemma 4.3 shows that SNS guarantees not only that the UARL are well behaved, but that the limits (4.37) exist and the $\sigma_{i}$ coincide with the orders of infinite zeros. Thus we may conclude that the unbounded closed-loop root loci terminate at the infinite zeros in the usual sense.

Remark 4.3. We assume in this section that SNS is satisfied, and show in the next that after applying the proposed controller (4.35) to the plant, the augmented system always satisfies SNS.

We now present some preliminary results for which it is possible to obtain a high gain approximation of the closed-loop system. First, we show that the application of controller 4.35 does not alter the orders of infinite zeros of $G(s)$.

Lemma 4.4. The orders of infinite zeros of $G(s)$ and $C(s, \epsilon) G(s)$ coincide.

Proof. Suppose $G(s)$ has orders of infinite zeros $\left\{\sigma_{1}, \ldots, \sigma_{v}\right\}$. Let $s=\frac{1}{z}$. This ensures that the SmithMcMillan form has the following form,

$$
D_{M}(G(z))=\left[\begin{array}{ccccc}
\frac{\epsilon_{1}(z)}{\psi_{1}(z)} & & & & \\
& \ddots & & & \\
& & \frac{\epsilon_{m-v}(z)}{\psi_{m-v}(z)} z^{\sigma_{1}} & & \\
& & & \ddots & \\
& & & & \frac{\epsilon_{m}(z)}{\psi_{m}(z)} z^{\sigma_{v}}
\end{array}\right]
$$

since by Definition 2.15 we necessarily have a factor of $z^{\sigma_{i}}$ in the $(m-v+i)^{t h}$ diagonal entry due to the presence of a $\sigma_{i}^{t h}$ order infinite zero. Notice the ordering since the numerator of the $i^{\text {th }}$ diagonal entry must divide the numerator of the $(i+1)^{\text {th }}$ diagonal entry.

Since the Smith-McMillan form of $C(z, \epsilon) G(z)$ is

$$
D_{M}(C(z, \epsilon) G(z))=c(z, \epsilon) D_{M}\left(U Y^{T} G(z)\right)
$$

by Theorem 4.2 we have

$$
\begin{aligned}
D_{M}(C(z, \epsilon) G(z)) & =c(z, \epsilon) D_{M}\left(U Y^{T}\right) D_{M}(G(z)) \\
& =c(z, \epsilon) D_{M}(G(z)) \\
& =\left[\begin{array}{rrrr}
c(z, \epsilon) \frac{\epsilon_{1}(z)}{\psi_{1}(z)} & & \\
& \ddots & & \\
& c(z, \epsilon) \frac{\epsilon_{m-v}(z)}{\psi_{m-v}(z)} z^{\sigma_{1}} & & \\
& & \ddots & \\
& & c(z, \epsilon) \frac{\epsilon_{m}(z)}{\psi_{m}(z)} z^{\sigma_{v}}
\end{array}\right] .
\end{aligned}
$$

We note that since $c(s, \epsilon)$ is non-strictly proper, $c(z, \epsilon)$ does not contribute any additional powers of $z$ to $z^{\sigma_{i}}$ in the $(m-v+i)^{t h}$ diagonal element of $D_{M}(G(z))$. This ensures that the orders of infinite zeros remain unchanged and the proof is complete.

It is the aim of the next series of results to determine, at high gain, which quantities in the structure defined by Lemma 4.1 dominate. Thus, a careful consideration of the orders of the quantities is required. 
Proposition 4.1. As $\epsilon \downarrow 0, s \rightarrow \infty$ according to the limits given in Definition 4.1, $c(s, \epsilon)$ as given in 4.35) is an $O\left(s^{q}\right)$ quantity where $q>l$, and $l$ is the the order of $c(s, \epsilon)$.

Proof.

$$
\begin{aligned}
\lim _{\epsilon \downarrow 0} \frac{1}{\epsilon} \frac{h(s)}{\prod_{j=1}^{l}\left(\epsilon^{p_{j}} s+1\right)} & =\lim _{\epsilon \downarrow 0} \frac{1}{\epsilon s^{\sigma_{i}}} \frac{s^{\sigma_{i}} h(s)}{\prod_{j=1}^{l}\left(\epsilon^{p_{j}} s+1\right)} \\
& \rightarrow \frac{1}{\left(\lambda_{i}+o(\epsilon)\right)} \frac{1}{(1+o(\epsilon))} O\left(s^{l+\sigma_{i}}\right) \\
& =O\left(s^{q}\right)
\end{aligned}
$$

for $\sigma_{i} \in\left\{1, \ldots, \sigma_{v}\right\}$ since by Lemma 4.4 no other limits exist.

Consider the series in Lemma 4.1. Denote $G_{i}(s)$ as the series beginning from the $(2,2)$ block of the $i^{\text {th }}$ term. $G_{1}(s)$ is then the entire series. Recall that, with controller 4.35 and $G_{1}(s)$ in mind, the closed-loop poles are given by the roots of

$$
\begin{aligned}
\Delta_{1}(s, \epsilon) & =\operatorname{det}\left(I_{m}+C(s, \epsilon) G(s)\right) \\
& =\operatorname{det}\left(I_{m}+c(s, \epsilon) U Y^{T} G(s)\right) \\
& =\operatorname{det}\left(I_{m}+c(s, \epsilon) Y^{T} G(s) U\right) \\
& =\operatorname{det}\left(I_{m}+c(s, \epsilon) G_{1}(s)\right) \\
& =0 .
\end{aligned}
$$

The indexing of the above definition alludes that an iteration over the $G_{i}(s)$ will be required. Due to the structure of $G_{1}(s)$ given by Lemma 4.1 we can compute the closed-loop poles by repeatedly applying Schur's formula for determinants. Thus, in the next step we show that $G_{i}(s)$ can be broken down into two separate components. Additionally, it is shown that some of the terms in these components become small in magnitude as $s$ becomes large.

Proposition 4.2. Let $c(s, \epsilon)$ be as in 4.35) and $G_{i}(s)$ as above. The following holds as $\epsilon \downarrow 0, s \rightarrow \infty$ according to Definition 4.1 if and only if $l \geq \sigma_{v}-2$.

$$
\operatorname{det}\left(I+c(s, \epsilon) G_{i}(s)+O\left(\frac{1}{s^{2}}\right)\right)=\Delta_{1}^{i}(s, \epsilon) \Delta_{2}^{i}(s, \epsilon)
$$

where

$$
\begin{aligned}
& \Delta_{1}^{i}(s, \epsilon)=\operatorname{det}\left(I+\frac{c(s, \epsilon)}{s^{\sigma_{i}}} \Sigma_{i}+\frac{c(s, \epsilon)}{s^{\sigma_{i}+1}} W_{i}(s)+O\left(\frac{1}{s^{2}}\right)\right) \\
& \Delta_{2}^{i}(s, \epsilon)=\operatorname{det}\left(I+c(s, \epsilon) G_{i+1}(s)+O\left(\frac{1}{s^{2}}\right)\right) .
\end{aligned}
$$

Proof. Consider the left-hand side of 4.51. It can be written as,

$$
\operatorname{det}\left(\left[\begin{array}{ll}
\alpha_{11}^{i}(s, \epsilon) & \alpha_{12}^{i}(s, \epsilon) \\
\alpha_{21}^{i}(s, \epsilon) & \alpha_{22}^{i}(s, \epsilon)
\end{array}\right]\right)
$$


where

$$
\begin{aligned}
& \alpha_{11}^{i}(s, \epsilon)=I+\frac{c(s, \epsilon)}{s^{\sigma_{i}}} \Sigma_{i}+\frac{c(s, \epsilon)}{s^{\sigma_{i}+1}} W_{i}(s)+O\left(\frac{1}{s^{2}}\right), \\
& \alpha_{12}^{i}(s, \epsilon)=\frac{c(s, \epsilon)}{s^{\sigma_{i}+1}} X_{i}(s)+O\left(\frac{1}{s^{2}}\right), \\
& \alpha_{21}^{i}(s, \epsilon)=\frac{c(s, \epsilon)}{s^{\sigma_{i}+1}} Y_{i}(s)+O\left(\frac{1}{s^{2}}\right), \\
& \alpha_{22}^{i}(s, \epsilon)=I+\frac{c(s, \epsilon)}{s^{\sigma_{i}+2}} Z_{i}(s)+c(s, \epsilon) G_{i+1}(s)+O\left(\frac{1}{s^{2}}\right) .
\end{aligned}
$$

Post multiply the first column of 4.54 by $A=\left[-\left(\frac{c(s, \epsilon)}{s^{\sigma_{i}}} \Sigma_{i}+\frac{c(s, \epsilon)}{s^{\sigma_{i}+1}} W_{i}(s)\right)^{-1} \frac{c(s, \epsilon)}{s^{\sigma_{i}+1}} X_{i}(s)\right]$ and add it to the second column. Namely,

$$
\begin{aligned}
\operatorname{det}\left(\left[\begin{array}{ll}
\alpha_{11}^{i}(s, \epsilon) & \alpha_{12}^{i}(s, \epsilon) \\
\alpha_{21}^{i}(s, \epsilon) & \alpha_{22}^{i}(s, \epsilon)
\end{array}\right]\right) & =\operatorname{det}\left(\left[\begin{array}{ll}
\alpha_{11}^{i}(s, \epsilon) & \alpha_{12}^{i}(s, \epsilon) \\
\alpha_{21}^{i}(s, \epsilon) & \alpha_{22}^{i}(s, \epsilon)
\end{array}\right]\right) \cdot \operatorname{det}\left(\left[\begin{array}{cc}
I & A \\
0 & I
\end{array}\right]\right) \\
& =\operatorname{det}\left(\left[\begin{array}{ll}
\alpha_{11}^{i}(s, \epsilon) & \alpha_{12}^{i}(s, \epsilon) \\
\alpha_{21}^{i}(s, \epsilon) & \alpha_{22}^{i}(s, \epsilon)
\end{array}\right]\left[\begin{array}{cc}
I & A \\
0 & I
\end{array}\right]\right) \\
& =\operatorname{det}\left(\left[\begin{array}{ll}
\alpha_{11}^{i}(s, \epsilon) & \beta_{1}^{i}(s, \epsilon) \\
\alpha_{21}^{i}(s, \epsilon) & \beta_{2}^{i}(s, \epsilon)
\end{array}\right]\right)
\end{aligned}
$$

where since $W_{i}(s), X_{i}(s), Y_{i}(s)$ are proper,

$$
\begin{aligned}
\beta_{1}^{i}(s, \epsilon)= & \left(I+\frac{c(s, \epsilon)}{s^{\sigma_{i}}} \Sigma_{i}+\frac{c(s, \epsilon)}{s^{\sigma_{i}+1}} W_{i}(s)+O\left(\frac{1}{s^{2}}\right)\right) \times\left[-\left(\frac{c(s, \epsilon)}{s^{\sigma_{i}}} \Sigma_{i}+\frac{c(s, \epsilon)}{s^{\sigma_{i}+1}} W_{i}(s)\right)^{-1} \frac{c(s, \epsilon)}{s^{\sigma_{i}+1}} X_{i}(s)\right] \\
& +\frac{c(s, \epsilon)}{s^{\sigma_{i}+1}} X_{i}(s)+O\left(\frac{1}{s^{2}}\right) \\
= & -\left(\frac{c(s, \epsilon)}{s^{\sigma_{i}}} \Sigma_{i}+\frac{c(s, \epsilon)}{s^{\sigma_{i}+1}} W_{i}(s)\right)^{-1} \frac{c(s, \epsilon)}{s^{\sigma_{i}+1}} X_{i}(s) \\
& -O\left(\frac{1}{s^{2}}\right)\left(\left(\frac{c(s, \epsilon)}{s^{\sigma_{i}}} \Sigma_{i}+\frac{c(s, \epsilon)}{s^{\sigma_{i}+1}} W_{i}(s)\right)^{-1} \frac{c(s, \epsilon)}{s^{\sigma_{i}+1}} X_{i}(s)\right)+O\left(\frac{1}{s^{2}}\right) \\
= & -\left(s \Sigma_{i}+W_{i}(s)\right)^{-1} X_{i}(s)-O\left(\frac{1}{s^{2}}\right)\left(s \Sigma_{i}+W_{i}(s)\right)^{-1} X_{i}(s)+O\left(\frac{1}{s^{2}}\right) \\
= & O\left(\frac{1}{s}\right)+O\left(\frac{1}{s^{3}}\right)+O\left(\frac{1}{s^{2}}\right) \\
= & O\left(\frac{1}{s}\right)
\end{aligned}
$$


and

$$
\begin{aligned}
\beta_{2}^{i}(s, \epsilon)= & \left(\frac{c(s, \epsilon)}{s^{\sigma_{i}+1}} Y_{i}(s)+O\left(\frac{1}{s^{2}}\right)\right) \times\left[-\left(\frac{c(s, \epsilon)}{s^{\sigma_{i}}} \Sigma_{i}+\frac{c(s, \epsilon)}{s^{\sigma_{i}+1}} W_{i}(s)\right)^{-1} \frac{c(s, \epsilon)}{s^{\sigma_{i}+1}} X_{i}(s)\right] \\
& +I+\frac{c(s, \epsilon)}{s^{\sigma_{i}+2}} Z_{i}(s)+c(s, \epsilon) G_{i+1}(s)+O\left(\frac{1}{s^{2}}\right) \\
= & I+c(s, \epsilon) G_{i+1}(s)+O\left(\frac{1}{s^{2}}\right)+O\left(\frac{1}{s^{2}}\right)\left[-\left(s \Sigma_{i}+W_{i}(s)\right)^{-1} X_{i}(s)\right] \\
= & I+c(s, \epsilon) G_{i+1}(s)+O\left(\frac{1}{s^{2}}\right) .
\end{aligned}
$$

Note the use of 4.21) for $\beta_{2}^{i}(s, \epsilon)$.

By using Schur's formula for determinants on 4.61 we see immediately that

$$
\Delta_{1}^{i}(s, \epsilon)=\operatorname{det} \alpha_{11}^{i}(s, \epsilon)
$$

and

$$
\Delta_{2}^{i}(s, \epsilon)=\operatorname{det}\left(\beta_{2}^{i}(s, \epsilon)-\alpha_{21}^{i}(s, \epsilon) \alpha_{11}^{i}(s, \epsilon)^{-1} \beta_{1}^{i}(s, \epsilon)\right)
$$

The matrix within $\Delta_{2}^{i}(s, \epsilon)$ is equal to

$$
\begin{aligned}
& \beta_{2}^{i}(s, \epsilon)-\frac{c(s, \epsilon)}{s^{\sigma_{i}+1}} Y_{i}(s) \alpha_{11}^{i}(s, \epsilon)^{-1} \beta_{1}^{i}(s, \epsilon)-O\left(\frac{1}{s^{2}}\right) \alpha_{11}^{i}(s, \epsilon)^{-1} \beta_{1}^{i}(s, \epsilon) \\
= & \beta_{2}^{i}(s, \epsilon)-O\left(\frac{c(s, \epsilon)}{s^{\sigma_{i}+1}}\right) O\left(\frac{s^{\sigma_{i}}}{c(s, \epsilon)}\right) O\left(\frac{1}{s}\right)-O\left(\frac{1}{s^{2}}\right) O\left(\frac{s^{\sigma_{i}}}{c(s, \epsilon)}\right) O\left(\frac{1}{s}\right) \\
= & \beta_{2}^{i}(s, \epsilon)-O\left(\frac{1}{s^{2}}\right)-O\left(\frac{1}{s^{3}}\right) O\left(\frac{1}{s^{q-\sigma_{i}}}\right) \\
= & I+c(s, \epsilon) G_{i+1}(s)+O\left(\frac{1}{s^{2}}\right) .
\end{aligned}
$$

where the last equality is obtained by using Proposition 4.1, and consideration of the following. We require that

$$
O\left(\frac{s^{\sigma_{i}}}{s^{q+3}}\right) \leq O\left(\frac{1}{s^{2}}\right)
$$

for the equality in the last step to hold. This occurs if and only if

$$
\begin{aligned}
& (\forall i=1, \ldots, v) q+3-\sigma_{i} \geq 2 \\
\Leftrightarrow & (\forall i=1, \ldots, v) q \geq \sigma_{i}-1 \\
\Leftrightarrow & l+1 \geq \sigma_{v}-1
\end{aligned}
$$

since by Proposition 4.1, $q>l$. Thus, $l$ must satisfy

$$
l \geq \sigma_{v}-2
$$

Since by Lemma 4.4 no other orders of infinite zeros, $\sigma_{i}$, exist the proof is complete. 
What we have just shown is that for the $i^{t h}$ system to which the $\sigma_{i}^{t h}$ order of infinite zero is associated, $G_{i}(s)$ can be decomposed into a product of two terms. One of which involves the $i+1^{t h}$ system, $G_{i+1}(s)$. This will be of significance later. We now show that the term associated with the $\sigma_{i}^{\text {th }}$ order of infinite zero can be further manipulated.

Proposition 4.3. Let $c(s, \epsilon)$ be as in 4.35), and $\Delta_{1}^{i}(s, \epsilon)$ be as in Proposition 4.2. The following holds as $\epsilon \downarrow 0, s \rightarrow \infty$ according to Definition 4.1 if and only if $l \geq \sigma_{v}-2$.

$$
\Delta_{1}^{i}(s, \epsilon)=\operatorname{det}\left(I+\frac{c(s, \epsilon)}{s^{\sigma_{i}}} \Sigma_{i}\right) \operatorname{det}\left(I+O\left(\frac{1}{s}\right)\right) .
$$

Proof. Since $\Sigma_{i}$ is diagonal and contains strictly positive values, the term $I+\frac{c(s, \epsilon)}{s^{\sigma_{i}}} \Sigma_{i}$ is invertible giving

$$
\Delta_{1}^{i}(s, \epsilon)=\operatorname{det}\left(I+\frac{c(s, \epsilon)}{s^{\sigma_{i}}} \Sigma_{i}\right) \gamma_{i}(s, \epsilon)
$$

where,

$$
\begin{aligned}
\gamma_{i}(s, \epsilon) & =\operatorname{det}\left(I+\left(I+\frac{c(s, \epsilon)}{s^{\sigma_{i}}} \Sigma_{i}\right)^{-1}\left(\frac{c(s, \epsilon)}{s^{\sigma_{i}+1}} W_{i}(s)+O\left(\frac{1}{s^{2}}\right)\right)\right) \\
& =\operatorname{det}\left(I+O\left(\frac{s^{\sigma_{i}}}{c(s, \epsilon)}\right) O\left(\frac{c(s, \epsilon)}{s^{\sigma_{i}+1}}\right)+O\left(\frac{s^{\sigma_{i}}}{c(s, \epsilon)}\right) O\left(\frac{1}{s^{2}}\right)\right) \\
& =\operatorname{det}\left(I+O\left(\frac{1}{s}\right)+O\left(\frac{s^{\sigma_{i}}}{s^{q+2}}\right)\right) \\
& =\operatorname{det}\left(I+O\left(\frac{1}{s}\right)\right)
\end{aligned}
$$

where the last equality is attained by considering the following. We require that

$$
O\left(\frac{s^{\sigma_{i}}}{s^{q+2}}\right) \leq O\left(\frac{1}{s}\right)
$$

for the equality in the last step to hold. This occurs if and only if

$$
\begin{aligned}
& (\forall i=1, \ldots, v) q+2-\sigma_{i} \geq 1 \\
\Leftrightarrow & (\forall i=1, \ldots, v) q \geq \sigma_{i}-1 \\
\Leftrightarrow & l+1 \geq \sigma_{v}-1
\end{aligned}
$$

since by Proposition 4.1, $q>l$. Thus, $l$ must satisfy

$$
l \geq \sigma_{v}-2 .
$$

Since by Lemma 4.4 no other orders of infinite zeros, $\sigma_{i}$, exist the proof is complete.

As $s \rightarrow \infty$ there are quantities in $\Delta_{1}^{i}(s, \epsilon)$ that are becoming arbitrarily small. We wish to ensure that these terms do not adversely affect the number of roots of the approximation. 
Proposition 4.4. Let $\Delta_{1}^{i}(s, \epsilon)$ be as in Proposition 4.2. Then $\Delta_{1}^{i}(s, \epsilon)$ and $\operatorname{det}\left(I+\frac{c(s, \epsilon)}{s^{\sigma_{i}}} \Sigma_{i}\right)$ have the same number of zeros.

Proof. For this, we look to apply a generalized version of Rouché's Theorem (see Appendix A) for finite dimensional operators of a single complex variable (e.g. transfer matrices).

Fix $\epsilon>0$. Consider,

$$
\begin{aligned}
\Delta_{1}^{i}(s, \epsilon) & =I+\frac{c(s, \epsilon)}{s^{\sigma_{i}}} \Sigma_{i}+\frac{c(s, \epsilon)}{s^{\sigma_{i}+1}} W_{i}(s)+O\left(\frac{1}{s^{2}}\right) \\
& =I+\frac{c(s, \epsilon)}{s^{\sigma_{i}}} \Sigma_{i}+c(s, \epsilon) P_{1}^{i T} C\left(\sum_{j=\sigma_{i}+1}^{\infty} \frac{A^{j-1}}{s^{j}}\right) B R_{1}^{i}+O\left(\frac{1}{s^{2}}\right) \\
& =I+\frac{c(s, \epsilon)}{s^{\sigma_{i}}} \Sigma_{i}+\frac{c(s, \epsilon)}{s^{\sigma_{i}}} P_{1}^{i T} C A^{\sigma_{i}}\left(\sum_{j=1}^{\infty} \frac{A^{j-1}}{s^{j}}\right) B R_{1}^{i}+O\left(\frac{1}{s^{2}}\right) \\
& =I+\frac{c(s, \epsilon)}{s^{\sigma_{i}}} \Sigma_{i}+\frac{c(s, \epsilon)}{s^{\sigma_{i}}} \tilde{C}^{i}\left(\sum_{j=1}^{\infty} \frac{A^{j-1}}{s^{j}}\right) \tilde{B}^{i}+O\left(\frac{1}{s^{2}}\right) \\
& =I+\frac{c(s, \epsilon)}{s^{\sigma_{i}}} \Sigma_{i}+\frac{c(s, \epsilon)}{s^{\sigma_{i}}} \tilde{C}^{i}\left(s I_{n}-A\right)^{-1} \tilde{B}^{i}+O\left(\frac{1}{s^{2}}\right) .
\end{aligned}
$$

where the constant matrices $P_{1}^{i}, R_{1}^{i}$ are determined by Lemma 4.1. To show the domination condition we have,

$$
\begin{aligned}
& \left\|\left(I+\frac{c(s, \epsilon)}{s^{\sigma_{i}}} \Sigma_{i}\right)^{-1}\left(\frac{c(s, \epsilon)}{s^{\sigma_{i}}} \tilde{C}^{i}(s I-A)^{-1} \tilde{B}^{i}+O\left(\frac{1}{s^{2}}\right)\right)\right\| \\
\leq & \left\|\left(\frac{s^{\sigma_{i}}}{c(s, \epsilon)} I+\Sigma_{i}\right)^{-1} \tilde{C}^{i}(s I-A)^{-1} \tilde{B}^{i}\right\|+\left\|\left(I+\frac{c(s, \epsilon)}{s^{\sigma_{i}}} \Sigma_{i}\right)^{-1} O\left(\frac{1}{s^{2}}\right)\right\| \\
\leq & \left\|\left(\frac{s^{\sigma_{i}}}{c(s, \epsilon)} I+\Sigma_{i}\right)^{-1}\right\| \cdot\left\|\tilde{C}^{i}(s I-A)^{-1} \tilde{B}^{i}\right\|+\left\|\left(I+\frac{c(s, \epsilon)}{s^{\sigma_{i}}} \Sigma_{i}\right)^{-1}\right\| \cdot\left\|O\left(\frac{1}{s^{2}}\right)\right\| .
\end{aligned}
$$

Define a simple, closed contour $\Gamma=r e^{j \theta}$ for $r>0$, and let $s \in \Gamma$. Since $c(r, \epsilon)$ is non-strictly proper in $r$ and thus approaches a constant as $r$ becomes sufficiently large, we can always make

$$
\left\|\left(\frac{r^{\sigma_{i}}}{c(r, \epsilon)} I+\Sigma_{i}\right)^{-1}\right\| \cdot\left\|\tilde{C}^{i}(r I-A)^{-1} \tilde{B}^{i}\right\|+\left\|\left(I+\frac{c(r, \epsilon)}{r^{\sigma_{i}}} \Sigma_{i}\right)^{-1}\right\| \cdot\left\|O\left(\frac{1}{r^{2}}\right)\right\|<1
$$

by making the radius of $\Gamma$ sufficiently large. Therefore $\Delta_{1}^{i}(s, \epsilon)$ and $\operatorname{det}\left(I+\frac{c(s, \epsilon)}{s^{\sigma_{i}}} \Sigma_{i}\right)$ have the same number of roots.

Using the above, a high gain approximation of the closed-loop system is given in the following lemma and is the main result of this section. Although the statement is different, the idea of the result was originally proposed by Zhang [34].

Lemma 4.5. Suppose $G(s) \in \mathbb{R}(s)^{m \times m}$, and $C(s, \epsilon)$ is given by 4.35). The unbounded roots of $\operatorname{det}\left(I_{m}+\right.$ $C(s, \epsilon) G(s))$, and $\prod_{i=1}^{v} \operatorname{det}\left(I+\frac{c(s, \epsilon)}{s^{\sigma_{i}}} \Sigma_{i}\right)$ coincide as $\epsilon \downarrow 0$, where $\Sigma_{i}, \sigma_{i}$ are given in Lemma 4.1 if and only if $l \geq \sigma_{v}-2$. 
Proof. The idea behind the proof is to decompose the system based on its infinite zero structure using Lemma 4.1 so that we may perform further analysis.

For strictly proper systems as is the case here, some of the branches of the characteristic polynomial tend to infinity. Thus we seek to approximate the behaviour of those infinite branches as $\epsilon \downarrow 0$, and $s \rightarrow \infty$. The behaviour associated with $\epsilon$, and $s$ associated with each branch are characterized by Lemma 4.3 .

Consider the first order of infinite zeros $\sigma_{1}$. Then the closed-loop characteristic polynomial is equal to

$$
\begin{aligned}
\operatorname{det}\left(I_{m}+C(s, \epsilon) G(s)\right) & =\operatorname{det}\left(I_{m}+c(s, \epsilon) U Y^{T} G(s)\right) \\
& =\operatorname{det}\left(I_{m}+c(s, \epsilon) Y^{T} G(s) U\right) \\
& =\operatorname{det}\left(I_{m}+c(s, \epsilon) G_{1}(s)\right) \\
& =0
\end{aligned}
$$

where,

$$
G_{1}(s)=\left[\begin{array}{cc}
\frac{1}{s^{\sigma_{1}}} \Sigma_{1}+\frac{1}{s^{\sigma_{1}+1}} W_{1}(s) & \frac{1}{s^{\sigma_{1}+1}} X_{1}(s) \\
\frac{1}{s^{\sigma_{1}+1}} Y_{1}(s) & \frac{1}{s^{\sigma_{1}+2}} Z_{1}(s)+\frac{1}{s^{\sigma_{2}}} G_{2}(s)
\end{array}\right]
$$

$G_{2}(s)$ is the rest of the series given by Lemma 4.1. By Proposition 4.2 ,

$$
\operatorname{det}\left(I+c(s, \epsilon) G_{1}(s)\right)=\Delta_{1}^{1}(s, \epsilon) \Delta_{2}^{1}(s, \epsilon)
$$

where

$$
\begin{aligned}
& \Delta_{1}^{1}(s, \epsilon)=\operatorname{det}\left(I+\frac{c(s, \epsilon)}{s^{\sigma_{1}}} \Sigma_{1}+\frac{c(s, \epsilon)}{s^{\sigma_{1}+1}} W_{1}(s)+O\left(\frac{1}{s^{2}}\right)\right), \\
& \Delta_{2}^{1}(s, \epsilon)=\operatorname{det}\left(I+c(s, \epsilon) G_{2}(s)+O\left(\frac{1}{s^{2}}\right)\right) .
\end{aligned}
$$

This can be verified by setting the $O\left(\frac{1}{s^{2}}\right)$ term to zero and following the development of Proposition 4.2 Next, by Proposition 4.3 we have that

$$
\Delta_{1}^{1}(s, \epsilon)=\operatorname{det}\left(I+\frac{c(s, \epsilon)}{s^{\sigma_{1}}} \Sigma_{1}\right) \operatorname{det}\left(I+O\left(\frac{1}{s}\right)\right) .
$$

Additionally, by Proposition $4.4 \Delta_{1}^{1}(s, \epsilon)$ and $\operatorname{det}\left(I+\frac{c(s, \epsilon)}{s^{\sigma_{1}}} \Sigma_{1}\right)$ have the same number of roots, i.e. ensuring that no roots are "lost" due to the approximation.

Since $G_{2}(s)$ has the same structure as $G_{1}(s)$, we can repeatedly apply Propositions $4.2,4.3$, and 4.4 to obtain

$$
\operatorname{det}\left(I_{m}+c(s, \epsilon) U Y^{T} G(s)\right)=\left[\prod_{j=1}^{v} \operatorname{det}\left(I_{d_{j}}+\frac{c(s, \epsilon)}{s^{\sigma_{j}}} \Sigma_{j}\right)\right]\left[\prod_{j=1}^{v} \operatorname{det}\left(I_{d_{j}}+O\left(\frac{1}{s}\right)\right)\right] .
$$

Letting $\epsilon \downarrow 0$, and $s \rightarrow \infty$ proves the claim.

Remark 4.4. By the choice of $l$ (the order of $c(s, \epsilon)$ in (4.35)) the high gain decomposition given in 
Lemma 4.5 is what enables the design of low order controllers. $l$ is only a function of the highest order of infinite zero of the system, which for most practical systems is quite low.

Lemma 4.5 provides the means to apply the results developed in the previous chapter. At high gain, the open loop system can be decomposed into several SISO integrator systems of different order. We know precisely how to handle this situation where a single controller is required to stabilize a family of systems of different relative degrees from Theorem 3.1

\subsection{Simple Null Structure}

It is a possibility of the multivariable root locus to have Butterworth patterns that do not correspond to the orders of infinite zeros of the system. In particular, the orders of the Butterworth patterns need not be integers [10, 1. An example is given in Chapter 5. It has been shown in [1] that the orders of the various Butterworth patterns that emerge coincide with the orders of infinite zeros if and only Simple Null Structure (SNS, see Definition 2.20 is satisfied.

Remark 4.5. SNS is a generic system property in the sense that systems which do not possess SNS lie in a set of measure zero [1].

Much of the literature on multivariable root locus assumes that the system satisfies the SNS assumption [10, 14, 15. We show in this section that the proposed controller 4.35) ensures that the augmented plant satisfies SNS.

Lemma 4.6. Consider controller 4.35), and let $G(s) \in \mathbb{R}(s)^{m \times m}$. The open loop system composed of the controller and $G(s)$ ensures that the augmented system $G^{\prime}(s)=Y^{T} G(s) U$ satisfies SNS.

Proof. The open loop system is

$$
O L S(s, \epsilon)=c(s, \epsilon) U Y^{T} G(s)
$$

or equivalently by considering that $\operatorname{det}\left(I+c(s, \epsilon) U Y^{T} G(s)\right)=\operatorname{det}\left(I+c(s, \epsilon) Y^{T} G(s) U\right)$,

$$
\begin{aligned}
O L S(s, \epsilon) & =c(s, \epsilon) Y^{T} G(s) U \\
& =c(s, \epsilon) G^{\prime}(s) .
\end{aligned}
$$

It is sufficient to show that all the Projected Markov Parameters of $G^{\prime}(s)=Y^{T} G(s) U$ have simple canonical blocks for the 0 eigenvalue, i.e. the dimension of the eigenspace associated with 0 is equal to the number of 0 eigenvalues.

However, it is immediate from Lemma 4.1 that since $U, Y$ are exactly those matrices which perform the singular value decomposition on each PMP of $G(s)$, the $i^{\text {th }} \mathrm{PMP}$ of $G^{\prime}(s)$ is then given by

$$
G_{i}=\left[\begin{array}{cc}
\Sigma_{i} & 0 \\
0 & 0_{d_{i}}
\end{array}\right]
$$

Correspondingly, $\Sigma_{i}$ is a diagonal matrix consisting of the non-zero singular values of the $i^{\text {th }}$ PMP of $G(s)$ so that the $i^{\text {th }}$ PMP of $G^{\prime}(s)$, as given above, is always diagonal and therefore always satisfies SNS. Thus the proof is complete. 
Remark 4.6. The constant matrices $U, Y$ can be thought of rotations in the input and output space respectively to "align" the system in such a way that each of the PMPs are diagonal matrices with non-negative entries.

\subsection{High Gain Stabilization, the MIMO Case}

From the previous sections we can immediately imply the following results. Recall that the controller has the following structure

$$
C(s, \epsilon)=c(s, \epsilon) U Y^{T}
$$

where the dynamical portion of the controller is given by $(3.2)$, or $(3.61)$ and the constant matrices $U, Y$ are given by Lemma 4.1. Recall also that

$$
c(s, \epsilon)=\frac{1}{\epsilon^{p_{0}}} \frac{h(s)}{\prod_{i=1}^{l}\left(\epsilon^{p_{i}} s+1\right)}
$$

where $h(s)$ is a Hurwitz polynomial of degree $l$, and $p_{i} \in \mathbb{N}, i=0, \ldots, l$. The order of $c(s, \epsilon)$ is $l$, and denote $L$ as the order of 4.114. Assume that the plant $G(s)$ has orders of infinite zeros $\left\{\sigma_{1}, \ldots, \sigma_{v}\right\}$, where $1 \leq \sigma_{1}<\cdots<\sigma_{v}$. Finally, recall that we assume $G(s)$ is invertible and minimum phase through the entirety of the thesis.

Theorem 4.3. The controller 4.114) asymptotically stabilizes the system $G(s)$ (1.8) for sufficiently small $\epsilon$ if $l \geq \sigma_{v}-1$.

Proof. For sufficiently small $\epsilon$ we have that from Lemma 4.5, the unbounded closed-loop poles are equal to

$$
\operatorname{det}\left(I_{m}+C(s, \epsilon) G(s)\right)=\prod_{j=1}^{v} \operatorname{det}\left(I_{d_{j}}+\frac{c(s, \epsilon)}{s^{\sigma_{j}}} \Sigma_{j}\right) .
$$

The right-hand side is equivalent to a collection of decoupled SISO integrator systems of different size, all having positive instantaneous gain since $\Sigma_{i}$ is a diagonal matrix containing strictly positive entries. Thus, an application of Theorem 3.1, 3.2 or 3.3 will stabilize this family of SISO systems of bounded relative degree. Since the remaining poles remain finite as $\epsilon \downarrow 0$ and approach the transmission zeros of $G(s)$ which are stable by assumption, the proof is complete.

Theorem 4.4. The controller 4.114) asymptotically stabilizes the system $G(s)$ (1.8) for sufficiently small $\epsilon$ only if $l \geq \sigma_{v}-2$.

Proof. Follows immediately from the arguements within the proof of Theorem 4.3 and Corollary 3.1.

This of course implies the following.

Corollary 4.2. The controller (4.114) asymptotically stabilizes the system $G(s)$ (1.8) for sufficiently small $\epsilon$ only if the order of the controller, $L \geq m\left(\sigma_{v}-2\right)$.

The above results are essentially the culmination of the theoretical developments in this thesis. Stabilization by this method can be achieved by controller 4.114 given sufficiently high gain. The 
reader may have observed a gap between the sufficiency result of Theorem 4.3 and the necessity result of Theorem 4.4. This gap originates from Theorem 3.1 and Corollary 3.1 in Chapter 3 . An intuitive explanation of this is as follows. Consider the standard SISO root locus problem for a system of relative degree two. The unbounded poles then form a Butterworth pattern of order $\sigma=2$ so that the asymptotes are parallel to the imaginary axis. However, we still cannot determine if the unbounded poles are stable at high gain. We need the additional information about the real axis intersection point of the asymptotes in order to determine this. The theory in Chapter 3 does not provide a method to calculate the analagous point in our (more complicated) case. Thus we observe the gap between sufficiency and necessity.

The structure of the controller is only a function of the highest order of infinite zero of the system, i.e. $\sigma_{v}$. In particular, to achieve stabilization, the lower bound on the order of the controller is also a function of $\sigma_{v}$. From this bound, we see that if the orders of infinite zeros are small it is possible to design controllers that stabilize the closed-loop system which are of low order. As will be seen in Chapter 5. most practical systems indeed have small orders of infinite zeros. In contrast, if the designer were to use an optimal control technique such as the cheap control framework of Chapter 2 , the order of the controller is fixed to be the order of the system itself. Thus, a low order controller is obtained only if the order of the system is small. The results in this section show that if the designer studies the infinite zero structure of the system and allow high gain control, the order of the controller can be reduced.

\subsection{Perfect Regulation with Bounded Peaking}

We are now ready to solve the perfect regulation problem with bounded peaking using our proposed approach. Unlike much of the previous developments, perfect regulation with bounded peaking follows from directly verifying Kimura's conditions, i.e. Theorem 2.14 from Chapter 2 We show that the conditions hold with the proposed controller. Recall that $l$ is a parameter of controller 4.35), and for transfer matrix $G(s) \in \mathbb{R}(s)^{m \times m}$ suppose that $G(s)$ has orders of infinite zeros $\left\{\sigma_{1}, \ldots, \sigma_{v}\right\}$ where $1 \leq \sigma_{1}<\cdots<\sigma_{v}$.

Theorem 4.5. The controller (4.35) achieves perfect regulation with bounded peaking for the system (1.8) if $l \geq \sigma_{v}-1$.

Proof. Since we have chosen the $l \geq \sigma_{v}-1$, closed-loop stability is established by Theorem 4.3 . Let $T_{x}(s, \epsilon)$ be as in 1.12 , i.e.

$$
T_{x}(s, \epsilon)=T(s, \epsilon) W_{x}(s)
$$

where

$$
\begin{aligned}
T(s, \epsilon) & =\left[I_{m}-G(s) C(s, \epsilon)\right]^{-1}, \\
W_{x}(s) & =C\left(s I_{n}-A\right)^{-1} .
\end{aligned}
$$

The controller $C(s, \epsilon)$ is given by 4.35 , and $A, B, C$ are the matrices in the state space realization of $G(s)$. It is required to show that as $\epsilon \downarrow 0$

$$
\begin{aligned}
T_{x}(s, \epsilon) & \rightarrow 0, \\
T_{x}\left(\frac{s}{\epsilon}, \epsilon\right) & \rightarrow 0 .
\end{aligned}
$$


Since for $A \in \mathbb{R}(s)^{m \times m}$

$$
\|A\|=0 \Leftrightarrow A=0
$$

we shall show instead that as $\epsilon \downarrow 0$

$$
\begin{aligned}
\left\|T_{x}(s, \epsilon)\right\| & \rightarrow 0, \\
\left\|T_{x}\left(\frac{s}{\epsilon}, \epsilon\right)\right\| & \rightarrow 0 .
\end{aligned}
$$

Fix $s \in \mathbb{C}$. Since

$$
\lim _{\epsilon \downarrow 0}\|c(s, \epsilon)\|=\infty
$$

we have

$$
\begin{aligned}
\left\|T_{x}(s, \epsilon)\right\| & =\left\|\left[I_{m}-G(s) C(s, \epsilon)\right]^{-1} W_{x}(s)\right\| \\
& =\left\|\left[I_{m}-c(s, \epsilon) G(s) U Y^{T}\right]^{-1} W_{x}(s)\right\| \\
& \leq\left\|\left[I_{m}-c(s, \epsilon) G(s) U Y^{T}\right]^{-1}\right\|\left\|W_{x}(s)\right\| \\
& \rightarrow 0
\end{aligned}
$$

as $\epsilon \downarrow 0$. Next, with some straightforward manipulations, consider

$$
\begin{aligned}
\left\|T_{x}\left(\frac{s}{\epsilon}, \epsilon\right)\right\| & =\left\|\left[I_{m}-c\left(\frac{s}{\epsilon}, \epsilon\right) G\left(\frac{s}{\epsilon}\right) U Y^{T}\right]^{-1} C\left(\frac{s}{\epsilon} I_{n}-A\right)^{-1}\right\| \\
& =\left\|\left[I_{m}-\frac{1}{\epsilon^{p_{0}}} \frac{h\left(\frac{s}{\epsilon}\right)}{\prod_{i=1}^{l}\left(\epsilon^{p_{i}-1} s+1\right)} C\left(\frac{s}{\epsilon} I-A\right)^{-1} B U Y^{T}\right]^{-1} C\left(\frac{s}{\epsilon} I_{n}-A\right)^{-1}\right\| \\
& =\left\|\left[I_{m}-\frac{1}{\epsilon^{p_{0}-1}} \frac{h\left(\frac{s}{\epsilon}\right)}{\prod_{i=1}^{l}\left(\epsilon^{p_{i}-1} s+1\right)} C(s I-\epsilon A)^{-1} B U Y^{T}\right]^{-1} C\left(\frac{s}{\epsilon} I_{n}-A\right)^{-1}\right\| \\
& \leq\left\|\left[I_{m}-\frac{1}{\epsilon^{p_{0}-1}} \frac{h\left(\frac{s}{\epsilon}\right)}{\prod_{i=1}^{l}\left(\epsilon^{p_{i}-1} s+1\right)} C(s I-\epsilon A)^{-1} B U Y^{T}\right]^{-1}\right\|\left\|C\left(\frac{s}{\epsilon} I_{n}-A\right)^{-1}\right\| \\
& =\left\|\left[I_{m}-O\left(\frac{1}{\epsilon^{p_{0}-1}}\right) \frac{O\left(\frac{1}{\epsilon^{l}}\right)}{O(1)} O(1)\right]^{-1}\right\|\|O(\epsilon)\| \\
& =\|O(1)\|\|O(\epsilon)\| \\
& \rightarrow 0
\end{aligned}
$$

as $\epsilon \downarrow 0$. Note that the first term above is certainly finite but may not necessarily equal 0 . Consider for example $G(s)=\frac{1}{s}, p_{0}=1$, and $l=0$. Since the second term tends to zero, the proof is complete.

\subsection{Decentralized Control \& Perfect Regulation}

Using the theory and intuition developed in the previous sections, we wish to identify a class of linear, time-invariant systems for which it is possible to solve the perfect regulation problem with bounded peaking when restricted to decentralized control. Additionally, we seek to use the previous tools that 
are central to this thesis.

\subsubsection{Positive Instantaneous Gain}

In the SISO case, $G(s)=\frac{n(s)}{d(s)}$, positive instantaneous gain refers to the coefficient of the highest power of $s$ in the numerator polynomial $n(s)$ being a real positive scalar. We wish to make a similar definition for square, invertible, and minimum phase MIMO systems $G(s) \in \mathbb{R}(s)^{m \times m}$. In light of Lemma 4.5, and referring to Theorem 2.9. suppose that for $G(s) \in \mathbb{R}^{m \times m}$ each PMP is diagonalizable with non-negative real eigenvalues. Consider the following definition.

Definition 4.2. Let $G(s) \in \mathbb{R}(s)^{m \times m}$ be invertible. $G(s)$ is said to have positive instantaneous gain if for each $P M P G_{i}$ there exists non-singular $P_{i}$ which diagonalizes $G_{i}$ so that

$$
P_{i}^{-1} G_{i} P_{i}=\left[\begin{array}{cc}
\Lambda_{i} & 0 \\
0 & 0_{d_{i}}
\end{array}\right]
$$

and $\Lambda_{i} \subset \mathbb{R}_{++}$.

Remark 4.7. Note that under this definition, by applying the centralized controller (4.35) the open loop system always has positive instantaneous gain by Lemma 4.6.

\subsubsection{Perfect Decentralized Regulation with Bounded Peaking}

Suppose $G(s) \in \mathbb{R}(s)^{m \times m}$ is invertible, minimum phase, satisfies Simple Null Structure, and has positive instantaneous gain. Note that from Davison \& Wang [3] a minimum phase plant has no unstable decentralized fixed modes. Consider the following controller

$$
C(s, \epsilon)=c(s, \epsilon) I_{m}
$$

where the dynamical portion of the controller $c(s, \epsilon)$ is as in 3.2 , or 3.61). We then obtain the following lemma.

Lemma 4.7. Suppose $G(s) \in \mathbb{R}(s)^{m \times m}$ satisfies the assumptions above, and $C(s, \epsilon)$ is given by 4.138). The unbounded roots of $\operatorname{det}\left(I_{m}+C(s, \epsilon) G(s)\right)$ and $\prod_{i=1}^{v} \operatorname{det}\left(I+\frac{c(s, \epsilon)}{s^{\sigma_{i}}} \Lambda_{i}\right)$ have zeros that coincide as $\epsilon \downarrow 0$, where $\Lambda_{i}$ is a diagonal matrix containing the non-zero eigenvalues of the $i^{\text {th }} P M P$ of $G(s)$, and $\sigma_{i}$ is the ith order of infinite zero, if and only if $l \geq \sigma_{v}-2 . \sigma_{v}$ is the largest order of infinite zero.

Proof. Consider the closed-loop poles

$$
\begin{aligned}
\operatorname{det}\left(I_{m}+C(s, \epsilon) G(s)\right) & =\operatorname{det}\left(P^{-1} P+P^{-1} c(s, \epsilon) G(s) P\right) \\
& =\operatorname{det}\left(I_{m}+c(s, \epsilon) P^{-1} G(s) P\right) .
\end{aligned}
$$

By the positive instantaneous gain assumption, the eigenvalue decomposition is used since all PMPs of $G(s)$ are diagonalizable. Thus, $Y^{T}$ and $U$ of Lemma 4.1 are replaced by $P^{-1}$ and $P$ respectively. Thus the proof is complete by applying Lemma 4.5 .

Remark 4.8. Intuitively, the positive instantaneous gain assumption ensures that the collection of SISO systems from Lemma 4.7 have positive instantaneous gain in the usual SISO sense. The case of negative 
instantaneous gain can be avoided by introducing a negative sign into the controller 4.138). When there is a mix of positive and negative real eigenvalues in some PMP of $G(s)$ there will accordingly be a mix of positive and negative instantaneous gains in the collection of SISO systems. This would then cause at least one of the SISO systems to become unstable as $\epsilon \downarrow 0$. The case where some eigenvalues are complex is not well defined.

The following is a direct consequence of the previous sections.

Theorem 4.6. Suppose $G(s) \in \mathbb{R}(s)^{m \times m}$ is invertible, minimum phase, satisfies Simple Null Structure, and has positive instantaneous gain. The decentralized controller (4.138) achieves perfect regulation with bounded peaking if $l \geq \sigma_{v}-1$.

Proof. Since the system is minimum phase, $G(s)$ has no unstable decentralized fixed modes. The SNS assumption ensures that the orders of the Butterworth patterns formed by the unbounded root loci are equal to the orders of infinite zeros. This ensures that Lemma 4.7 remains valid. Thus stability is established by Theorem 4.3. Perfect regulation with bounded peaking is directly given by Theorem 4.5

Remark 4.9. Martensson [22] has a similar development as presented here. He introduces a different notion of positive instantaneous gain which we believe is somewhat cumbersome to verify but perhaps more general. Since we draw the connection between the controller design and the multivariable root locus, our definition follows naturally from the PMPs of the system.

The structure of the decentralized controller presented here, like the centralized case (see (4.35)), is only dependent on the orders of infinite zeros. For the cases where our assumptions hold, decentralized control is possible without notions of diagonal dominance [33, or to solve LMIs that are involved with the Youla parameterization based methods [27].

The nature of the decentralized controller is simple in structure and of low dynamic order, however in exchange for this simplicity we have confined ourselves to a somewhat narrow class of systems since the positive instantaneous gain assumption is quite restrictive. SNS may be generic [1], but diagonalizability is not for matrices over the reals which is required by the positive instantaneous gain assumption. 


\section{Chapter 5}

\section{Case Studies}

The purpose of this chapter is to demonstrate the most significant aspects of the theory developed in this thesis. We have obtained a controller structure which solves the perfect regulation problem with bounded peaking that depends only on the infinite zero structure of the system as given in Chapter 4 .

Since we have proposed a one-parameter family of controllers, a design procedure for systems which satisfy our assumptions could be the following. Construct the controller 4.35 based on the orders of infinite zeros of the plant. Decrease $\epsilon$ until the plant is stable. If performance is satisfactory, stop. If an over-damped response is needed, lower $\theta$ (as defined in (3.61)), and re-tune $\epsilon$ until the desired performance is achieved.

We will illustrate in several different examples various aspects of the theory and how they may be used. The first example is of a linearized industrial chemical plant of $41^{\text {st }}$ order which demonstrates the potential savings in the order of the controller. Next, we describe a situation which belongs to the gap between Theorems 4.3 and 4.4 The third explores the case when the system does not satisfy Simple Null Structure, and how to overcome this issue with Lemma 4.6. The fourth demonstrates the use of Theorem 3.3 where the design incorporates all the orders of infinite zeros instead of only the largest one. The last study gives a solution to the decentralized perfect regulation problem with bounded peaking on the 2-linked inverted pendulum system, and illustrates the use of Theorem 3.2 to force an over-damped response.

\subsection{Industrial Chemical Plant}

We show the effectiveness of our approach by applying the proposed controller to a 4-input, 4-output, $41^{\text {st }}$ order chemical plant (see [31]). The chemical plant has 36 transmission zeros, all of which have negative real parts so that the system is minimum phase. To determine if the system has Simple Null Structure we use Theorem 2.9 to calculate the Projected Markov Parameters to be the following

$$
G_{1}=\left[\begin{array}{cccc}
3.36 & 0 & 0 & 0 \\
0 & 0 & 0 & 0 \\
0 & 0 & 0.01 & 0 \\
0 & 0 & 0 & -3.8 \times 10^{-6}
\end{array}\right], \quad G_{2}=30895 .
$$

The zero eigenvalue in $G_{1}$ is not repeated and $G_{2}$ is non-singular which implies that Simple Null 


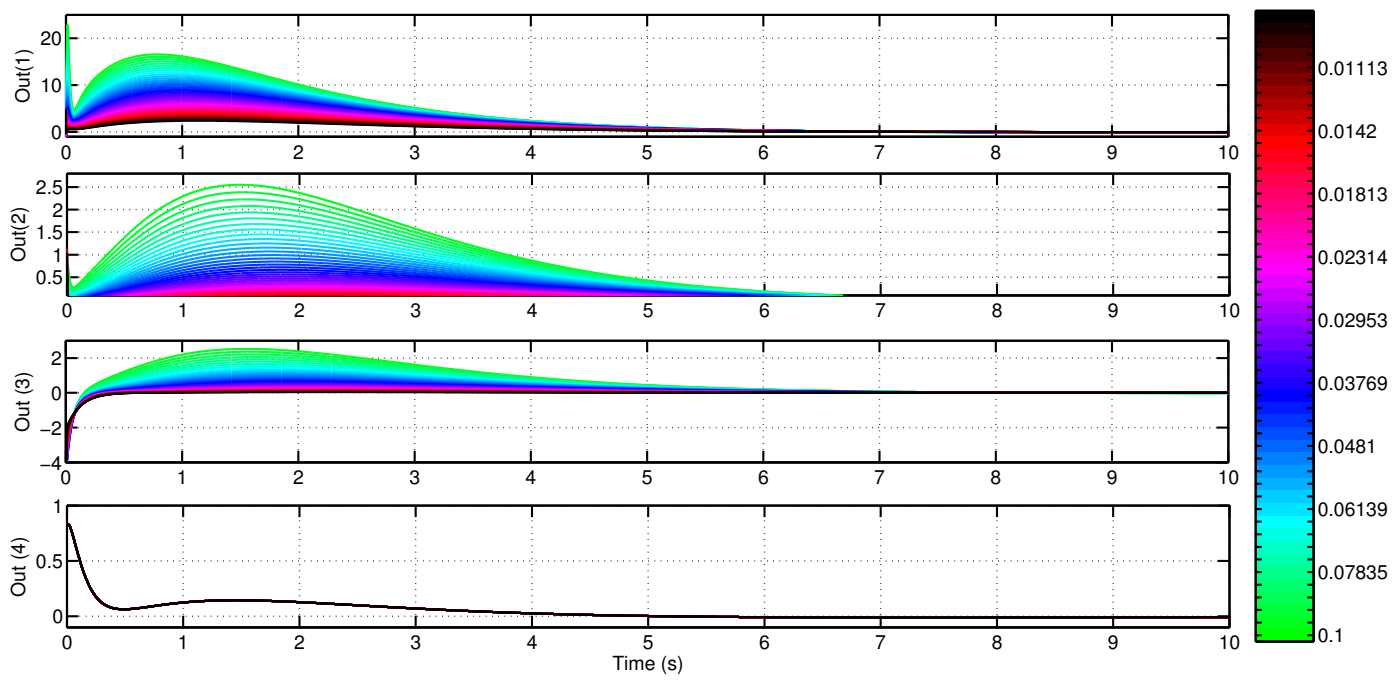

Figure 5.1: Initial condition response of chemical plant. Note that our choice of gains were not large enough to significantly change the response in the fourth output.

Structure is satisfied. Since the sequence of PMPs has terminated $\left(\right.$ at $\left.G_{2}\right)$, we know by Lemma 4.2 that the system is invertible. All of our assumptions are thus satisfied.

By Theorem 4.3 the resulting controller 4.35 has dynamic order 4. To synthesize the controller, matrices $U, Y$ from Lemma 4.1 are needed and computed as follows.

The matrices $U, Y$ are obtained in much the same way as the diagonalizing matrices used to develop the multivariable root locus in Chapter 2 (see Theorem 2.9. . The difference here is that Lemma 4.1 uses the singular value decomposition (SVD) rather than the eigenvalue decomposition. Thus, small modifications need to be made. We proceed with the SVD of the first PMP, $G_{1}$,

$$
G_{1}=P_{1} \Sigma_{1} Q_{1}^{T}=\left[\begin{array}{cccc}
1 & 0 & 0 & 0 \\
0 & 0 & 0 & 1 \\
0 & 1 & 0 & 0 \\
0 & 0 & 1 & 0
\end{array}\right]\left[\begin{array}{cccc}
3.36 & 0 & 0 & 0 \\
0 & 0.01 & 0 & 0 \\
0 & 0 & -3.8 \times 10^{-6} & 0 \\
0 & 0 & 0 & 0
\end{array}\right]\left[\begin{array}{cccc}
1 & 0 & 0 & 0 \\
0 & 0 & 1 & 0 \\
0 & 0 & 0 & -1 \\
0 & 1 & 0 & 0
\end{array}\right]
$$

The rank defect of $G_{1}$ is then $d_{1}=1$ so that (by Theorem 2.10 there are $1\left(m-d_{1}\right)=1(4-1)=3$ first order infinite zeros. Since the second PMP $G_{2}=30895$ is non-singular, we have $d_{2}=0$ so that there are $2\left(d_{1}-d_{2}\right)=2(1-0)=2$ second order infinite zeros. In addition, the trivial SVD of $G_{2}$ is

$$
G_{2}=P_{2} \Sigma_{2} Q_{2}^{T}=1 \times 30895 \times 1
$$


Therefore, the matrices $U, Y$ from Lemma 4.1 are

$$
\begin{aligned}
& Y^{T}=\left[\begin{array}{cc}
I_{m-d_{1}} & 0 \\
0 & P_{2}^{T}
\end{array}\right] P_{1}^{T}, \\
& U=Q_{1}\left[\begin{array}{cc}
I_{m-d_{1}} & 0 \\
0 & Q_{2}
\end{array}\right]
\end{aligned}
$$

so that the final controller is

$$
\begin{aligned}
C(s, \epsilon) & =c(s, \epsilon) U Y^{T} \\
& =c(s, \epsilon) \times\left[\begin{array}{cccc}
1 & 0 & 0 & 0 \\
0 & 0 & 0 & 1 \\
0 & 1 & 0 & 0 \\
0 & 0 & -1 & 0
\end{array}\right]\left[\begin{array}{cccc}
1 & 0 & 0 & 0 \\
0 & 0 & 1 & 0 \\
0 & 0 & 0 & 1 \\
0 & 1 & 0 & 0
\end{array}\right] \\
& =\frac{1}{\epsilon} \frac{s+1}{\epsilon s+1} \times\left[\begin{array}{cccc}
1 & 0 & 0 & 0 \\
0 & 1 & 0 & 0 \\
0 & 0 & 1 & 0 \\
0 & 0 & 0 & -1
\end{array}\right] .
\end{aligned}
$$

When compared to an observer based approach, the resulting controller would be $41^{\text {st }}$ order, the same dimensionality as the plant. Even if a reduced order observer is designed where a state transformation is applied to manipulate the $C$ matrix, a $37^{t h}$ order controller is expected. We plot the multiple responses to a uniformly random initial condition $x(0)$ to varying $\epsilon$ in Figure 5.1 . Notice that the responses become faster and increasingly well-behaved as $\epsilon$ becomes small.

\subsection{An Example in the Gap}

Consider the system $G(s)=\frac{1}{s^{3}}$. Theorem 4.3 states that choosing the order $l \geq 2$ of the controller 4.35 is sufficient for stabilization. On the other hand, Theorem 4.4 indicates that the order must satisfy $l \geq 1$ for stabilization to be possible.

Let $l=1$, i.e. the controller for $p_{0}=p_{1}=1$ is given by

$$
c(s, \epsilon)=\frac{1}{\epsilon} \frac{s+1}{\epsilon s+1} .
$$

Controller 5.9 is an example which satisfies the necessary condition however does not stabilize the closed-loop system. By using the NP diagram approach (see Chapter 3), it is easily verified that the relevant link equations are

$$
\begin{aligned}
& c_{1}^{2}+1=0, \\
& c_{2}+1=0
\end{aligned}
$$

which would indicate that of the three asymptotes, two would form a line parallel to the imaginary axis, and the remainder would make an angle $\pi$ with the positive real axis. The problem can be identified by simply plotting the root loci in Figure 5.2 . 


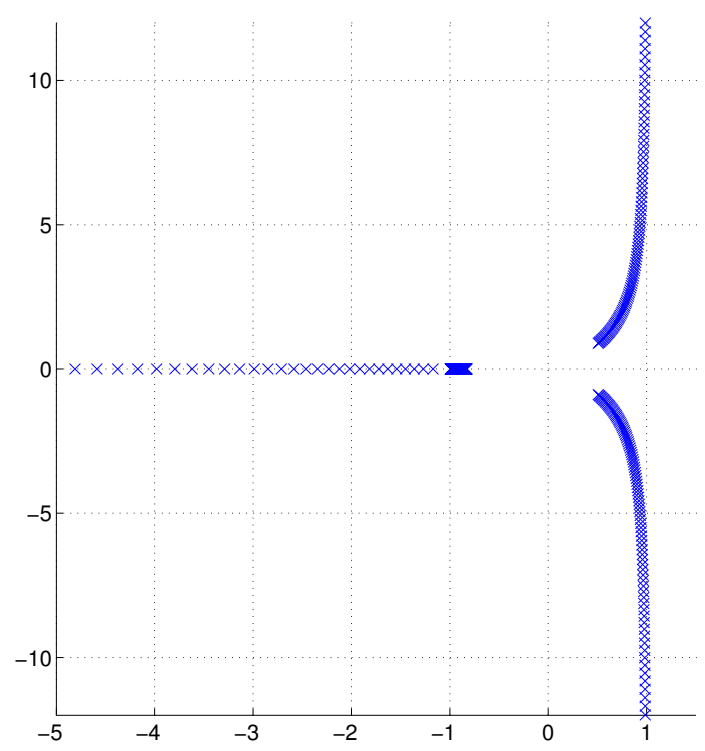

Figure 5.2: Root locus of the closed-loop poles.

The real axis intercept point for the asymptotes that are parallel to the imaginary axis is roughly at $w_{a}=+1$ (determined experimentally from Figure 5.2 which would cause the instability of the closedloop system as $\epsilon \downarrow 0$. The sufficient condition for stability avoids this issue altogether since by choosing $l \geq r^{*}-1=2$ and the $\left\{p_{i}\right\}$ accordingly, the angles of the asymptotes will always point into $\mathbb{C}_{-}$. It is also clear that if the necessary condition is violated here, i.e. $l=0$, the closed-loop system would, by inspection, be unstable for sufficiently small $\epsilon$ by classical root locus arguments.

\subsection{A Violation of Simple Null Structure}

An investigation of the behaviour of a system that has Non-simple Null Structure is given (see Definition 2.20 . In particular, we highlight the genericity of the SNS property, and show how Non-simple Null Structure can be dealt with by Lemma 4.6 .

Consider the following system from Hung \& MacFarlane [10]

$$
G(s)=\left[\begin{array}{ccc}
0 & \frac{1}{s} & 0 \\
0 & 0 & \frac{1}{s^{2}} \\
\frac{1}{s^{2}} & 0 & 0
\end{array}\right]
$$

and its Projected Markov Parameters

$$
G_{1}=\left[\begin{array}{lll}
0 & 1 & 0 \\
0 & 0 & 0 \\
0 & 0 & 0
\end{array}\right], \quad G_{2}=\left[\begin{array}{cc}
0 & -1 \\
1 & 0
\end{array}\right]
$$

Since $G_{1}$ has a Jordan block of size 2 for the 0 eigenvalue, SNS is not satisified. If we compute the 
closed-loop poles of $\operatorname{det}\left(I+\frac{1}{\epsilon} G(s)\right)=1+\frac{1}{\epsilon^{3} s^{5}}=0$ giving $s=\left(\frac{-1}{\epsilon}\right)^{\frac{3}{5}}$. We thus have a Butterworth pattern of order $\frac{3}{5}$ which clearly cannot coincide with any order of infinite zero since it is a rational number.

Naturally, by the structure of $G(s)$ a simple reordering of the inputs would decouple $G(s)$ and remove the Non-simple Null Structure property.

If we design the controller 4.35 we obtain

$$
\begin{aligned}
C(s, \epsilon) & =c(s, \epsilon) U Y^{T} \\
& =c(s, \epsilon)\left[\begin{array}{lll}
0 & 0 & 1 \\
1 & 0 & 0 \\
0 & 1 & 0
\end{array}\right]\left[\begin{array}{lll}
1 & 0 & 0 \\
0 & 1 & 0 \\
0 & 0 & 1
\end{array}\right]
\end{aligned}
$$

so that since the closed-loop poles are roots of

$$
\operatorname{det}\left(I+c(s, \epsilon) U Y^{T} G(s)\right)=\operatorname{det}\left(I+c(s, \epsilon) Y^{T} G(s) U\right)
$$

the open loop system then becomes

$$
\begin{aligned}
O L S(s, \epsilon) & =c(s, \epsilon) Y^{T} G(s) U \\
& =c(s, \epsilon)\left[\begin{array}{ccc}
\frac{1}{s} & 0 & 0 \\
0 & \frac{1}{s^{2}} & 0 \\
0 & 0 & \frac{1}{s^{2}}
\end{array}\right] .
\end{aligned}
$$

Thus, by the application of the constant matrices $U, Y$ as given by Lemma 4.1, the augmented system now satisfies SNS as predicted by Lemma 4.6 since the PMPs of $Y^{T} G(s) U$ are diagonal and given by

$$
G_{1}=\left[\begin{array}{lll}
1 & 0 & 0 \\
0 & 0 & 0 \\
0 & 0 & 0
\end{array}\right], \quad G_{2}=\left[\begin{array}{ll}
1 & 0 \\
0 & 1
\end{array}\right]
$$

\subsection{Designing For the Orders of Infinite Zeros}

We explore in this section how the designer may choose to utilize Theorem 3.3. It is demonstrated that if one directly considers all of the orders of infinite zeros, a significant reduction in the gain of the controller may be attained. Consider the system

$$
G(s)=\frac{1}{s^{5}} .
$$

This system belongs to the family of systems $G_{5}$ (see (3.1) which in total has orders of infinite zeros equal to $\{1,2,3,4,5\}$.

Although we consider a SISO system here, the same results apply for MIMO systems as well since MIMO systems in general have multiple orders of infinite zeros. 
The static gain of the controller 3.2 can be verified to be

$$
\frac{1}{\epsilon^{\sum_{j=0}^{l} p_{j}}}
$$

where in this case $l=4$. By Theorem 3.1 the smallest set of $\left\{p_{0}, p_{1}, p_{2}, p_{3}, p_{4}\right\}$ is $\{1,1,2,4,8\}$ (compare Martensson's $\{1,2,4,8,16\}$ [22] ) since we are forced to also stabilize systems with relative degree less than 5 as well. We define $\Gamma$ (see (3.71)), and $G_{\Gamma}$ (see $(3.72)$ ) as follows

$$
\begin{aligned}
\Gamma & =\{5\}, \\
G_{\Gamma} & =\left\{G(s) \in G_{5}: r=5\right\}
\end{aligned}
$$

where $r$ is the relative degree of $G(s)$, and $G_{5}$ is given by (3.1). From Theorem 3.3, we can obtain the same result, i.e. stabilization, with $\{1,1,2,3,4\}$ by explicitly considering that only the $5^{\text {th }}$ order of infinite zero is present in $G_{\Gamma}$.

It is also clear that the gain of the controller 3.2 grows exponentially in the highest order of infinite zero by (5.21). Thus from a practical point of view, if the highest order of infinite zero (or for the case of SISO systems, the relative degree) is large, then a high gain approach is necessary when using the proposed approach. Fortunately, most real-world systems have relatively low orders of infinite zeros.

\subsection{Decentralized Control of 2-Linked Inverted Pendulum}

In light of the results in Chapter 4 on decentralized control, we provide a practical example to solve the decentralized perfect regulation problem with bounded peaking. Consider the following linearization of a 2-linked inverted pendulum system

$$
\begin{aligned}
& \dot{x}=\left[\begin{array}{cccc}
0 & 1 & 0 & 0 \\
9.8 & 0 & -9.8 & 0 \\
0 & 0 & 0 & 1 \\
-9.8 & 0 & 29.4 & 0
\end{array}\right] x+\left[\begin{array}{cc}
0 & 0 \\
1 & -2 \\
0 & 0 \\
-2 & 5
\end{array}\right] u, \\
& y=\left[\begin{array}{llll}
1 & 0 & 0 & 0 \\
0 & 0 & 1 & 0
\end{array}\right] x
\end{aligned}
$$

which has the following PMPs

$$
G_{1}=0_{2} \quad G_{2}=\left[\begin{array}{cc}
1 & -2 \\
-2 & 5
\end{array}\right] .
$$

Thus the system has no first order infinite zeros, and 4 second order infinite zeros by Theorem 2.10

Taking the eigenvalue decomposition of $G_{2}$ we have

$$
G_{2}=P\left[\begin{array}{cc}
0.17157 & 0 \\
0 & 5.28284
\end{array}\right] P^{-1}
$$

where

$$
P=\left[\begin{array}{cc}
-0.92388 & -0.38268 \\
-0.38268 & 0.92388
\end{array}\right] .
$$




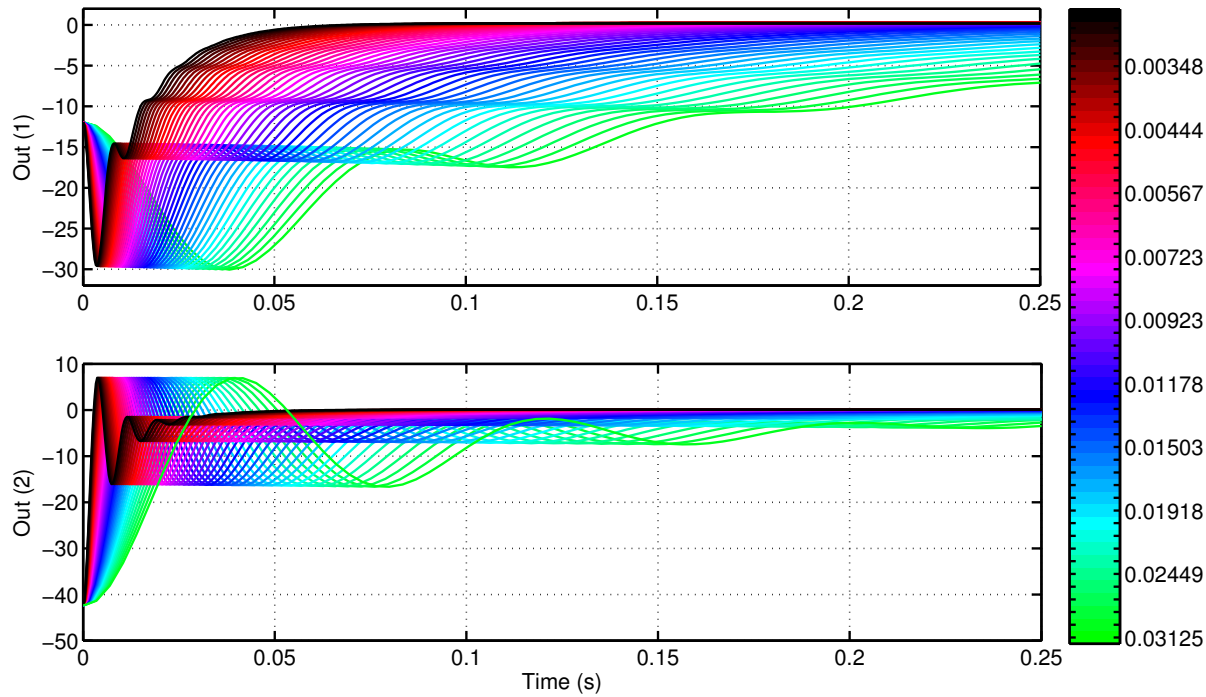

Figure 5.3: Initial condition response for the decentralized control of 2-linked inverted pendulum.

Thus, the 2-linked inverted pendulum system satisfies SNS, is minimum phase (has an absence of transmission zeros), is invertible by Lemma 4.2 , and has positive instantaneous gain since $\{0.17157,5.28284\} \subset$ $\mathbb{R}_{++}$.

Thus by Theorem 4.6 the following controller solves the decentralized perfect regulation problem with bounded peaking

$$
C(s, \epsilon)=\frac{1}{\epsilon} \frac{s+1}{\epsilon s+1} \times\left[\begin{array}{ll}
1 & 0 \\
0 & 1
\end{array}\right]
$$

which gives the following response in Figure 5.3 for varying $\epsilon$.

We now illustrate how Theorem 3.2 may be used to produce a well-damped response for the closedloop system by forcing all the unbounded closed-loop poles to go to infinity along the negative real axis. This is equivalent to forcing all the asymptotes of the root locus to lie on the negative real axis.

Consider the controller (3.61)

$$
C(s, \epsilon)=\frac{\theta}{\epsilon} \frac{s+1}{\epsilon s+1} \times\left[\begin{array}{ll}
1 & 0 \\
0 & 1
\end{array}\right]
$$

where $\theta=\{10,0.01\}$, and $\epsilon=0.0001$. We give a table of the closed-loop poles below.

Table 5.1: The closed-loop poles of the 2-linked inverted pendulum system by adjusting the parameter $\theta$.

\begin{tabular}{|l|l|l|}
\cline { 2 - 3 } \multicolumn{1}{c|}{} & $\theta=10$ & $\theta=0.01$ \\
\hline \multirow{5}{*}{ CLS Poles } & $-4.9995 e+03+7.6180 e+04 i$ & $-9.9828 e+03$ \\
& $-4.9995 e+03-7.6180 e+04 i$ & $-9.3786 e+03$ \\
& $-4.9995 e+03+1.2107 e+04 i$ & $-6.2045 e+02$ \\
& $-4.9995 e+03-1.2107 e+04 i$ & $-1.6492 e+01$ \\
& $-9.9972 e-01$ & $-6.9346 e-01$ \\
& $-9.9994 e-01$ & $-9.4413 e-01$ \\
\hline
\end{tabular}


The adjustment of $\theta$ always allows the designer to achieve this behaviour. 


\section{Chapter 6}

\section{Concluding Remarks}

\subsection{Summary of Results}

Throughout this thesis, we have shown that for invertible, minimum phase systems, it is always possible to construct a controller of the form 4.35 to solve the perfect regulation problem with bounded peaking. In this way, we can regulate the output of a system arbitrarily fast, while ensuring the output remains bounded. The approach is highly dependent on the structural property known as the orders of infinite zeros which is intimately tied to the multivariable root locus. We have shown that the order of the proposed controller can be taken to be one less than the highest order of infinite zero of the system. Thus, a controller which solves the perfect regulation problem with bounded peaking can be made low order if the orders of infinite zeros of the system are small.

To achieve this, a generalization of a high gain stabilization result from 22 is derived, and also extended to the MIMO case in Chapters 3 , and 4 . Under conditions of positive instantaneous gain, and Simple Null Structure, decentralized control using our approach is possible and shown in Chapter 4 The case studies in Chapter 5 have demonstrated on practical systems how simple the controller, and design process is as well as the potential savings on the order of the controller.

\subsection{Future Work}

Future work in this direction could be to investigate less restrictive controller design under the decentralized information constraint. Since this constraint imposes enormous difficulty in the traditional design procedure, this would be a formidable task. To the best of the author's knowledge, with the current theory presented in this thesis there is no other obvious way to achieve decentralized control besides what is presented.

Another area of extension would be to determine the intersection point of the asymptotes under the proposed control 4.35). As shown in the second case study in Chapter 5 the theory currently does not predict where this point would be in the complex plane. It is of significance since, as seen in the case study, it can be a cause of instability.

Determining the intersection point of of the asymptotes would also lead to perhaps a tightening of the gap between sufficiency and necessity in Theorems 4.3 and 4.4 . Determining necessary and sufficient conditions would certainly illustrate a more complete picture of the overall investigation. 


\subsection{Final Thoughts}

We have shown that if one explicitly considers certain structural properties of a system, i.e. the infinite zero structure, one can design low order controllers which solve the perfect regulation problem with bounded peaking. To do this, we generalized a high-gain stabilization result from Martensson [22], and showed how it can be used in the multivariable case. We have also given a lower bound on the dynamic order for which the controller must satisfy. Since most practical systems, such as the ones given in Chapter 5, have small orders of infinite zeros, the potential decrease in the order of the controller when compared with observer-based techniques can be dramatic if one is willing to allow high-gain control. 


\section{Appendix A}

\section{A generalization of Rouché's Theorem}

We follow the developments of Gohberg \& Sigal [9] which generalize Rouché's Theorem to infinite dimensional operators of a single complex variable. We show that the results in [9] can be specialized to the finite dimensional case in Proposition A.1.

Let $\mathcal{B}$ be a Banach space, $\mathcal{U}(\mathcal{B})=\mathcal{U}$ be the algebra of all bounded linear operators in $\mathcal{B}$. Denote $\mathcal{U}\left(\lambda_{0}\right)=\mathcal{U}\left(\lambda_{0} ; \mathcal{B}\right)$ as the set of all operator-valued functions $A(\lambda)$ with values in $\mathcal{U}$ which are holomorphic in some neighbourhood of $\lambda_{0}$, except possibly at $\lambda_{0}$ itself. Denote also, $\mathcal{U}_{0}\left(\lambda_{0}\right)=\mathcal{U}_{0}\left(\lambda_{0} ; \mathcal{B}\right)$ to be the group of all operator-valued functions $A(\lambda) \in \mathcal{U}\left(\lambda_{0}\right)$ for which $\lambda_{0}$ is a regular point.

Definition A.1. The point $\lambda_{0}$ is a characteristic value of $A(\lambda) \in \mathcal{U}\left(\lambda_{0}\right)$ if there exists a vectorvalued function $\phi(\lambda)$ with values in $\mathcal{B}$, holomorphic at $\lambda_{0}$ with $\phi\left(\lambda_{0}\right) \neq 0$, such that the vector-valued function $A(\lambda) \phi(\lambda)$ is holomorphic at $\lambda_{0}$ and vanishes at this point. We call $\phi(\lambda)$ a root function of $A(\lambda)$ corresponding to $\lambda_{0}$.

Definition A.2. Let $\lambda_{0}$ be a characteristic value of the operator-valued function $A(\lambda)$ and $\phi(\lambda)$ its corresponding root function. The order of $\lambda_{0}$ as a zero of $A(\lambda) \phi(\lambda)$ is called the multiplicity of $\phi(\lambda)$, and the vector $\phi_{0}=\phi\left(\lambda_{0}\right)$ an eigenvector of $A(\lambda)$ corresponding to $\lambda_{0}$. The closure of the set of all eigenvectors of $A(\lambda)$ corresponding to $\lambda_{0}$ is called the kernel of $A(\lambda)$ corresponding to $\lambda_{0}$, and is denoted $\operatorname{Ker} A\left(\lambda_{0}\right)$.

Definition A.3. The rank of an eigenvector $\phi_{0}$ is the maximum of the multiplicities of all root functions $\phi(\lambda)$ such that $\phi\left(\lambda_{0}\right)=\phi_{0}$, if the set of these multiplicities is bounded. Otherwise the rank is taken to be infinity.

If the dimension of $\operatorname{Ker} A\left(\lambda_{0}\right)$ is of finite dimension $\alpha<\infty$ then let the integers

$$
r_{j}=\operatorname{rank} \phi_{0}^{j}
$$

for $j=1, \ldots, \alpha$ where $\operatorname{Ker} A\left(\lambda_{0}\right)=c l\left\{\phi_{0}^{1}, \ldots, \phi_{0}^{\alpha}\right\}$.

Definition A.4. The null multiplicity of the characteristic value $\lambda_{0}$ of $A(\lambda)$ is $N\left(A\left(\lambda_{0}\right)\right)=\sum_{j=1}^{\alpha} r_{j}$.

Definition A.5. The polar multiplicity of the characteristic value $\lambda_{0}$ of $A(\lambda)$ is $P\left(A\left(\lambda_{0}\right)\right)=N\left(A^{-1}\left(\lambda_{0}\right)\right)$. 
Definition A.6. The multiplicity of the characteristic value $\lambda_{0}$ of $A(\lambda)$ is $\mathcal{M}\left(A\left(\lambda_{0}\right)\right)=N\left(A\left(\lambda_{0}\right)\right)$ $P\left(A\left(\lambda_{0}\right)\right)$.

Theorem A.1. (Operator Rouché [9]) Let $A(\lambda)$ be an operator-valued function which is normal with respect to a simple closed rectifiable contour $\Gamma$ bounding a domain $G$. If an operator-valued function $S(\lambda)$ which is finitely meromorphic in $G$ and continous at $\Gamma$ satisfies the condition

$$
\left\|A^{-1}(\lambda) S(\lambda)\right\|<1 \quad(\lambda \in \Gamma)
$$

then $B(\lambda)=A(\lambda)+S(\lambda)$ is also normal with respect to $\Gamma$, and

$$
\mathcal{M}(B(\lambda) ; \Gamma)=\mathcal{M}(A(\lambda) ; \Gamma) .
$$

Proposition A.1. Suppose $A(\lambda) \in \mathcal{U}$ is invertible and consider the case when $\mathcal{B}=\mathbb{R}(\lambda)^{m \times m}$. Then,

$$
\mathcal{M}\left(A\left(\lambda_{0}\right)\right)=\mathcal{M}\left(\operatorname{det}\left(A\left(\lambda_{0}\right)\right)\right)
$$

Proof: We will require the following results:

1. $\mathcal{M}\left(A\left(\lambda_{0}\right)\right)=\frac{1}{2 \pi j} \operatorname{tr} \int_{\left|\lambda-\lambda_{0}\right|=\rho} A^{-1} \frac{d}{d \lambda} A(\lambda) d \lambda$ for $\rho$ sufficiently small.

2. For $A(\lambda)=E(\lambda) D(\lambda) F(\lambda)$ where $E, F \in \mathcal{U}_{0}\left(\lambda_{0}\right)$. Then,

$$
\mathcal{M}\left(A\left(\lambda_{0}\right)\right)=\mathcal{M}\left(E\left(\lambda_{0}\right) D\left(\lambda_{0}\right) F\left(\lambda_{0}\right)\right)=\mathcal{M}\left(D\left(\lambda_{0}\right)\right) .
$$

Since $A(\lambda)$ is finite dimensional with elements that are rational scalar functions of $\lambda$, we obtain the following decomposition by way of the Smith-McMillan form:

$$
A(\lambda)=E(\lambda) D(\lambda) F(\lambda)
$$

where $E, F \in \mathcal{U}_{0}\left(\lambda_{0}\right)$ and,

$$
\begin{aligned}
D(\lambda) & =\left[\begin{array}{ccc}
\left(\lambda-\lambda_{0}\right)^{n_{1} \frac{\delta_{1}(\lambda)}{\epsilon_{1}(\lambda)}} & 0 \\
& \ddots & \\
0 & & \left(\lambda-\lambda_{0}\right)^{n_{v}} \frac{\delta_{v}(\lambda)}{\epsilon_{v}(\lambda)}
\end{array}\right] \\
& =\left[\begin{array}{ccc}
\left(\lambda-\lambda_{0}\right)^{n_{1}} g_{1}(\lambda) & 0 \\
0 & \ddots & \left(\lambda-\lambda_{0}\right)^{n_{v}} g_{v}(\lambda)
\end{array}\right]
\end{aligned}
$$

where $\left(\lambda-\lambda_{0}\right)^{n_{i}}$ and $g_{i}(\lambda)$ are co-prime, and $n_{i}$ are integers for $i=1, \ldots, v$. Because $\mathcal{M}\left(A\left(\lambda_{0}\right)\right)=$ $\mathcal{M}\left(D\left(\lambda_{0}\right)\right)$ we then have,

$$
\mathcal{M}\left(A\left(\lambda_{0}\right)\right)=\frac{1}{2 \pi j} \operatorname{tr} \int_{\left|\lambda-\lambda_{0}\right|=\rho} D^{-1} \frac{d}{d \lambda} D(\lambda) d \lambda
$$


where $\rho$ is taken sufficiently small such that $\lambda_{0}$ is the only characteristic value or pole in $\left\{\lambda:\left|\lambda-\lambda_{0}\right| \leq \rho\right\}$. Some manipulation shows that,

$$
\begin{aligned}
\frac{d}{d \lambda} D(\lambda) & =\operatorname{diag}\left[n_{i}\left(\lambda-\lambda_{0}\right)^{n_{i}-1} g_{i}(\lambda)+\left(\lambda-\lambda_{0}\right)^{n_{i}} \frac{d}{d \lambda} g_{i}(\lambda)\right]_{i=1, \ldots, v} \\
D^{-1}(\lambda) & =\operatorname{diag}\left[\left(\lambda-\lambda_{0}\right)^{-n_{i}} \frac{1}{g_{i}(\lambda)}\right]_{i=1, \ldots, v}
\end{aligned}
$$

Then A.9 evaluates to,

$$
\begin{aligned}
\frac{1}{2 \pi j} \operatorname{tr} \int_{\left|\lambda-\lambda_{0}\right|=\rho} D^{-1} \frac{d}{d \lambda} D(\lambda) d \lambda & =\frac{1}{2 \pi j} \operatorname{tr} \int_{\left|\lambda-\lambda_{0}\right|=\rho}\left[\begin{array}{ccc}
\frac{n_{1}}{\lambda-\lambda_{0}}+\frac{g_{1}^{\prime}(\lambda)}{g_{1}(\lambda)} & \\
& \ddots & 0 \\
0 & & \frac{n_{v}}{\lambda-\lambda_{0}}+\frac{g_{v}^{\prime}(\lambda)}{g_{v}(\lambda)}
\end{array}\right] d \lambda \\
& =\sum_{i=1}^{v} n_{i} \times n\left(\Gamma ; \lambda_{0}\right) \\
& =\sum_{i=1}^{v} n_{i} \\
& =\mathcal{M}\left(\operatorname{det}\left(D\left(\lambda_{0}\right)\right)\right) \\
& =\mathcal{M}\left(\operatorname{det}\left(A\left(\lambda_{0}\right)\right)\right) .
\end{aligned}
$$




\section{Bibliography}

[1] C.I. Byrnes and P.K. Stevens. The mcmillan and newton polygons of a feedback system and the construction of root loci. International Journal of Control, 35(1):29-53, 1982.

[2] B.M. Chen, K. Liu, and Z. Lin. Solvability conditions and solutions to perfect regulation problem under measurement output feedback. Systems \& Control Letters, 40(4):269-277, 2000.

[3] E.J. Davison and S.H. Wang. A characterization of decentralized fixed modes in terms of transmission zeros. IEEE Transactions on Automatic Control, 30(1):81-82, 1985.

[4] W.R. Evans. Control system synthesis by root locus method. Transactions of the American Institute of Electrical Engineers, 69(1):66-69, 1950.

[5] B.A. Francis. The optimal linear-quadratic time-invariant regulator with cheap control. IEEE Transactions on Automatic Control, 24(4):616-621, 1979.

[6] B.A. Francis. Convergence in the boundary layer for singularly perturbed equations. Automatica, 18(1):57-62, 1982.

[7] B.A. Francis and K. Glover. Bounded peaking in the optimal linear regulator with cheap control. IEEE Transactions on Automatic Control, 23(4):608-617, 1978.

[8] B.A. Fuks. Functions of a Complex Variable and Some of Their Applications VOL. 2. Pergamon., 1961.

[9] I.C. Gohberg and E.I. Sigal. An operator generalization of the logarithmic residue theorem and the theorem of rouché. Sbornik: Mathematics, 13(4):603-625, 1971.

[10] Y.S. Hung and A.G.J. MacFarlane. On the relationships between the unbounded asymptote behaviour of multivariable root loci, impulse response and infinite zeros. International Journal of Control, 34(1):31-69, 1981.

[11] T. Kailath. Linear systems, volume 1. Prentice-Hall Englewood Cliffs, NJ, 1980.

[12] H. Kimura. A new approach to the perfect regulation and the bounded peaking in linear multivariable control systems. IEEE Transactions on Automatic Control, 26(1):253-270, 1981.

[13] P. Kokotovic, H.K. Khalil, and J. O'reilly. Singular perturbation methods in control: analysis and design, volume 25. SIAM, 1999.

[14] B. Kouvaritakis and J.M. Edmunds. Multivariate root loci: a unified approach to finite and infinite zeros. International Journal of Control, 29(3):393-428, 1979. 
[15] B. Kouvaritakis and U. Shaked. Asymptotic behaviour of root-loci of linear multivariable systems. International Journal of Control, 23(3):297-340, 1976.

[16] A.M. Krall. Stability techniques for continuous linear systems. CRC Press, 1967.

[17] B.C. Kuo. Automatic control systems. Prentice Hall PTR, 1981.

[18] H. Kwakernaak and R. Sivan. Linear optimal control systems, volume 1. Wiley-Interscience New York, 1972.

[19] H. Kwakernaak and R. Sivan. The maximally achievable accuracy of linear optimal regulators and linear optimal filters. IEEE Transactions on Automatic Control, 17(1):79-86, 1972.

[20] Z. Lin, A. Saberi, P. Sannuti, and Y. Shamash. Perfect regulation of linear multivariable systems a low-and-high-gain design. In Proceedings of Workshop on Advances in Control and its Applications, pages 173-192. Springer, 1996.

[21] S. Mac Lane and G. Birkhoff. Algebra. The MacMillan Company, 1968.

[22] B. Martensson. Adaptive Stabilization. PhD thesis, Lund Institute of Technology, 1986.

[23] M. Newman. On the smith normal form. J. Res. Nat. Bur. Standards Sect. B, 75:81-84, 1971.

[24] I. Newton. Geometria Analytica. 1680.

[25] I. Postlethwaite and A.G.J. MacFarlane. A complex variable approach to the analysis of linear multivariable feedback systems, volume 12. Springer-Verlag Berlin, 1979.

[26] V. Puiseux. Recherches sur les fonctions algébriques. 1850.

[27] M. Rotkowitz and S. Lall. A characterization of convex problems in decentralized control. IEEE Transactions on Automatic Control, 51(2):274-286, 2006.

[28] P. Sannuti. Direct singular perturbation analysis of high-gain and cheap control problems. Automatica, 19(1):41-51, 1983.

[29] P. Sannuti and H. Wason. A singular perturbation canonical form of invertible systems: determination of multivariate root-loci. International Journal of Control, 37(6):1259-1286, 1983.

[30] M. Vidyasagar. Control system synthesis: a factorization approach. Morgan \& Claypool Publishers, 2011.

[31] T.J. Williams and R.E. Otto. A generalized chemical processing model for the investigation of computer control. Transactions of the American Institute of Electrical Engineers, Part I: Communication and Electronics, 79(5):458-473, 1960.

[32] K. Young, P. Kokotovic, and V. Utkin. A singular perturbation analysis of high-gain feedback systems. IEEE Transactions on Automatic Control, 22(6):931-938, 1977.

[33] G. Zames and D. Bensoussan. Multivariable feedback, sensitivity, and decentralized control. IEEE Transactions on Automatic Control, 28(11):1030-1035, 1983. 
[34] H. Zhang and E.J. Davison. A uniform high-gain compensator for multivariable systems. In 33rd IEEE Conference on Decision and Control, Lake Buena Vista, Florida, 1994, Volume 1, pp. 892897. 